\title{
Metal-Catalyzed Silylene Transfer to Imines: Silaaziridine Synthesis and Reactivity
}

\author{
Zulimar Nevárez and K. A. Woerpel* \\ Department of Chemistry \\ University of California, Irvine \\ Irvine, CA 92697-2025
}

Supporting Information

\section{Contents:}

I. Thermal Silylene Transfer to Imine 12 S-2

II. AgOTf-Catalyzed Synthesis of Silaaziridines 3, 6a-e $\quad$ S-2

III. Isolation of Silaaziridines 3, 6a, \& 6c $\quad$ S-3

IV. Methanolysis of Silaaziridines 3, 6a-e $\quad$ S-4

V. AgOTf-Catalyzed Silylene Transfer to Conjugated Imines 8 \& $11 \quad$ S-6

VI. Electrophile Insertions of Silaaziridine $6 \mathbf{a} \quad$ S-8

VII. Palladium-Catalyzed Synthesis of Azasilacyclopentenes 16a-f and Control S-9

Experiment Supportive of Proposed Mechanism $\quad$ S-12

$\begin{array}{lr}\text { VIII. Protodesilylation of Azasilacyclopentene 16a } & \text { S-12 }\end{array}$

$\begin{array}{ll}\text { IX. References } & \text { S-13 }\end{array}$

$\begin{array}{llr}\text { X. X-ray Crystallographic Data } & \text { S-38 }\end{array}$

XI. Selected Spectra

General. Melting points were obtained using a Buchi 510 melting point apparatus and are reported uncorrected. Analytical thin layer chromatography was performed on EM reagents $0.25 \mathrm{~mm}$ silica gel $60-\mathrm{F}$ plates. Liquid chromatography was performed using force flow (Flash Chromatography) of the indicated solvent system on EM Reagents silica gel $\left(\mathrm{SiO}_{2}\right) 60$ (230-400 mesh). ${ }^{1} \mathrm{H}$ NMR and ${ }^{13} \mathrm{C}$ NMR spectra were recorded at $25{ }^{\circ} \mathrm{C} 400$ and 100 and 500 and $125 \mathrm{MHz}$ respectively, using Bruker DRX 400 and DRX 500 spectrometers as indicated. ${ }^{29} \mathrm{Si} \mathrm{NMR}$ spectra were recorded at $25{ }^{\circ} \mathrm{C}$ at $99.3 \mathrm{MHz}$. The ${ }^{1} \mathrm{H}$ NMR data are reported as follows: chemical shifts in ppm from internal tetramethylsilane or trimethylphenylsilane on the $\delta$ scale, multiplicity $(\mathrm{br}=$ broad, $\mathrm{s}=$ singlet, $\mathrm{d}=$ doublet, $\mathrm{t}=$ triplet, $\mathrm{q}=$ quartet, sext $=$ sextet, sept $=$ septet, oct $=$ octet, and $\mathrm{m}=$ multiplet), coupling constant $(\mathrm{Hz})$, and integration. The ${ }^{13} \mathrm{C} \mathrm{NMR}$ chemical shifts are reported in ppm from tetramethylsilane on the $\delta$ scale, using tetramethylsilane as an internal standard. The ${ }^{29} \mathrm{Si}$ NMR chemical shifts are reported in ppm from tetramethylsilane on the scale, using trimethylphenylsilane as an external standard. High-resolution mass spectra were acquired on an LCT Premier quadrupole time-of-flight spectrometer and were obtained by peak matching. IR samples of solids were prepared from concentrated solutions in dichloromethane. Microanalyses were performed by Atlantic Microlabs, Atlanta, GA or Desert Analytics, Tucson, AZ. Silacyclopropane $\mathbf{2}$ was stored and manipulated in an Innovative Technologies nitrogen atmosphere drybox and was prepared by known methods. ${ }^{1}$ All reaction mixtures to form silaaziridines, unless otherwise specified, were prepared in the drybox. All other reactions, unless specified were carried out under an atmosphere of nitrogen in glassware that had been flame-dried under a positive stream of nitrogen or under vacuum. Solvents were distilled or filtered before use. Unless otherwise noted, all reagents or substrates were commercially available. Imines were synthesized by known methods. ${ }^{2}$ 


\section{Thermal Silylene Transfer to Imine 1}

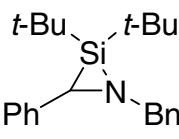

3

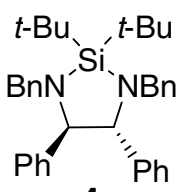

4

Silaaziridine 3 and Dimer 4. To a medium-wall NMR tube were added imine $1\left(0.25 \mathrm{~mL}, 0.46 \mathrm{M}^{\text {in }} \mathrm{C}_{7} \mathrm{D}_{8}\right.$, $0.12 \mathrm{mmol})$, silacyclopropane $2\left(0.25 \mathrm{~mL}, 0.46 \mathrm{M}\right.$ in $\left.\mathrm{C}_{7} \mathrm{D}_{8}, 0.12 \mathrm{mmol}\right)$, and $\mathrm{PhSiMe}_{3}(0.25 \mathrm{~mL}$ from silacyclopropane 2 stock solution, $0.092 \mathrm{M}, 0.023 \mathrm{mmol})$. The reaction mixture was degassed $(3 \mathrm{x}$ freezepump-thaw) and sealed under vacuum. The sealed NMR tube was placed in a $100{ }^{\circ} \mathrm{C}$ oil bath and monitored by ${ }^{1} \mathrm{H}$ NMR spectroscopy. After $12 \mathrm{~h}$, a mixture of silaaziridine $\mathbf{3}$ and dimer $\mathbf{4}$ were formed. Silaaziridine $\mathbf{3}$ was formed in $44 \%$ yield as determined by ${ }^{1} \mathrm{H}$ NMR spectroscopy: ${ }^{1} \mathrm{H}$ NMR $\left(\mathrm{C}_{7} \mathrm{D}_{8}, 500 \mathrm{MHz}\right.$, distinctive peaks $) \delta$ $4.53(\mathrm{~d}, J=13.0,1 \mathrm{H}), 3.92(\mathrm{~d}, J=13.0,1 \mathrm{H}), 3.40(\mathrm{~s}, 1 \mathrm{H}), 0.99(\mathrm{~s}, 9 \mathrm{H}), 0.89(\mathrm{~s}, 9 \mathrm{H})$. Complete characterization data for this compound are provided in section III (p. 4). Dimer 4 was formed in $27 \%$ yield as determined by ${ }^{1} \mathrm{H}$ NMR spectroscopy: ${ }^{1} \mathrm{H}$ NMR $\left(\mathrm{C}_{7} \mathrm{D}_{8}, 500 \mathrm{MHz}\right.$, distinctive peaks) $\delta 4.39(\mathrm{~s}, 1 \mathrm{H}), 4.23(\mathrm{~d}, J=15.3,1 \mathrm{H})$, $4.11(\mathrm{~d}, J=15.3,1 \mathrm{H}), 1.39(\mathrm{~s}, 9 \mathrm{H})$. Dimer 4, isolated by flash chromatography $\left(95: 5\right.$ hexanes: $\left.\mathrm{CH}_{2} \mathrm{Cl}_{2}\right)$ in $9 \%$ yield, exhibited spectral data that matched reported data for the known compound. ${ }^{3}$

\section{AgOTf-Catalyzed Synthesis of Silaaziridines 3 \& 6a-e}

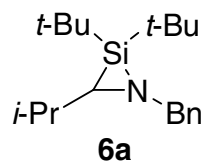

Representative Procedure for Metal-Catalyzed Silylene Transfer to Imines 1, 5a-e (NMR Experiment): Synthesis of Silaaziridines 3, 6a-e.

Silaaziridine 6a. To an NMR tube were added AgOTf $(0.3 \mathrm{mg}, 1 \mathrm{~mol} \%)$, imine 5a $\left(0.25 \mathrm{~mL}, 0.46 \mathrm{M}\right.$ in $\mathrm{C}_{7} \mathrm{D}_{8}$, $0.12 \mathrm{mmol})$, silacyclopropane $2\left(0.25 \mathrm{~mL}, 0.46 \mathrm{M}\right.$ in $\left.\mathrm{C}_{7} \mathrm{D}_{8}, 0.12 \mathrm{mmol}\right)$, and $\mathrm{PhSiMe}_{3}(0.25 \mathrm{~mL}$ from silacyclopropane 2 stock solution, $0.092 \mathrm{M}, 0.023 \mathrm{mmol}$ ). The reaction mixture was left at $23{ }^{\circ} \mathrm{C}$ and monitored by ${ }^{1} \mathrm{H}$ NMR spectroscopy. After $12 \mathrm{~h}$, silaaziridine 6a was formed in $88 \%$ yield as determined by ${ }^{1} \mathrm{H}$ NMR spectroscopy: $\quad{ }^{1} \mathrm{H}$ NMR $\left(\mathrm{C}_{7} \mathrm{D}_{8}, 500 \mathrm{MHz}\right.$, distinctive peaks) $\delta 4.41(\mathrm{~d}, J=13.5,1 \mathrm{H}), 3.99(\mathrm{~d}, J=13.5,1 \mathrm{H})$, $1.75(\mathrm{~d}, J=9.5,1 \mathrm{H}), 1.09(\mathrm{~s}, 9 \mathrm{H}), 0.96(\mathrm{~s}, 9 \mathrm{H}) ;{ }^{29} \mathrm{Si} \mathrm{NMR}\left(\mathrm{C}_{7} \mathrm{D}_{8}, 99.3 \mathrm{MHz}\right) \delta-46.5$. Complete characterization data for this compound are provided in section III (p. 3).

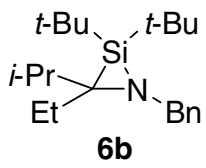

Silaaziridine 6b. The representative procedure for the synthesis of silaaziridines (NMR Experiment) was followed using $\operatorname{AgOTf}(0.3 \mathrm{mg}, 1 \mathrm{~mol} \%)$, imine $\mathbf{5 b}\left(0.25 \mathrm{~mL}, 0.46 \mathrm{M}\right.$ in $\left.\mathrm{C}_{6} \mathrm{D}_{6}, 0.12 \mathrm{mmol}\right)$, silacyclopropane 2 (0.50 mL, $0.46 \mathrm{M}$ in $\left.\mathrm{C}_{6} \mathrm{D}_{6}, 0.23 \mathrm{mmol}\right)$, and $\mathrm{PhSiMe}_{3}(0.50 \mathrm{~mL}$ from silacyclopropane 2 stock solution, 0.092 M, $0.046 \mathrm{mmol})$. After $24 \mathrm{~h}$, silaaziridine $\mathbf{6 b}$ was formed in $74 \%$ yield as determined by ${ }^{1} \mathrm{H}$ NMR spectroscopy: ${ }^{1} \mathrm{H}$ NMR $\left(\mathrm{C}_{6} \mathrm{D}_{6}, 400 \mathrm{MHz}\right.$, distinctive peaks) $\delta 4.23(\mathrm{~s}, 2 \mathrm{H}), 2.30,(\mathrm{~m}, 1 \mathrm{H}), 2.07(\mathrm{~m}, 1 \mathrm{H}), 1.08(\mathrm{~s}, 18 \mathrm{H}) ;{ }^{29} \mathrm{Si}$ $\operatorname{NMR}\left(\mathrm{C}_{6} \mathrm{D}_{6}, 99.3 \mathrm{MHz}\right) \delta-47.8$.

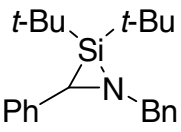

3

Silaaziridine 3. The representative procedure for the synthesis of silaaziridines (NMR Experiment) was followed using AgOTf (0.3 mg, $1 \mathrm{~mol} \%)$, imine 1 ( $0.25 \mathrm{~mL}, 0.46 \mathrm{M}$ in $\left.\mathrm{C}_{7} \mathrm{D}_{8}, 0.12 \mathrm{mmol}\right)$, silacyclopropane 2 (0.25 mL, 0.46 M in $\left.\mathrm{C}_{7} \mathrm{D}_{8}, 0.12 \mathrm{mmol}\right)$, and $\mathrm{PhSiMe}_{3}(0.25 \mathrm{~mL}$ from silacyclopropane 2 stock solution, 0.092 
M, $0.023 \mathrm{mmol})$. After $6 \mathrm{~h}$, silaaziridine 3 was formed in $90 \%$ yield as determined by ${ }^{1} \mathrm{H}$ NMR spectroscopy: ${ }^{1} \mathrm{H}$ NMR $\left(\mathrm{C}_{7} \mathrm{D}_{8}, 500 \mathrm{MHz}\right.$, distinctive peaks) $\delta 4.54(\mathrm{~d}, J=13.1,1 \mathrm{H}), 3.92(\mathrm{~d}, J=13.0,1 \mathrm{H}), 3.40(\mathrm{~s}, 1 \mathrm{H}), 0.99$ $(\mathrm{s}, 9 \mathrm{H}), 0.89(\mathrm{~s}, 9 \mathrm{H}) ;{ }^{29} \mathrm{Si} \mathrm{NMR}\left(\mathrm{C}_{7} \mathrm{D}_{8}, 99.3 \mathrm{MHz}\right) \delta-46.4$. Complete characterization data for this compound is provided in section III (p. 4).

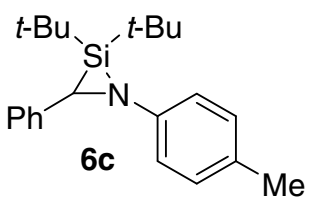

Silaaziridine 6c. The representative procedure for the synthesis of silaaziridines (NMR Experiment) was followed using AgOTf (0.3 mg, $1 \mathrm{~mol} \%)$, imine 5c $\left(0.25 \mathrm{~mL}, 0.46 \mathrm{M}\right.$ in $\left.\mathrm{C}_{7} \mathrm{D}_{8}, 0.12 \mathrm{mmol}\right)$, silacyclopropane 2 (0.25 mL, $0.46 \mathrm{M}$ in $\left.\mathrm{C}_{7} \mathrm{D}_{8}, 0.12 \mathrm{mmol}\right)$, and $\mathrm{PhSiMe}_{3}(0.25 \mathrm{~mL}$ from silacyclopropane 2 stock solution, 0.092 M, $0.023 \mathrm{mmol})$. After $1 \mathrm{~h}$, silaaziridine $6 \mathrm{c}$ was formed in $74 \%$ yield as determined by ${ }^{1} \mathrm{H}$ NMR spectroscopy: ${ }^{1} \mathrm{H}$ NMR $\left(\mathrm{C}_{7} \mathrm{D}_{8}, 500 \mathrm{MHz}\right.$, distinctive peaks) $\delta 3.81(\mathrm{~s}, 1 \mathrm{H}), 2.19(\mathrm{~s}, 3 \mathrm{H}), 0.99(\mathrm{~s}, 9 \mathrm{H}), 0.95(\mathrm{~s}, 9 \mathrm{H}) ;{ }^{29} \mathrm{Si} \mathrm{NMR}$ $\left(\mathrm{C}_{7} \mathrm{D}_{8}, 99.3 \mathrm{MHz}\right) \delta-46.3$. Complete characterization data for this compound is provided in section III (p. 4).

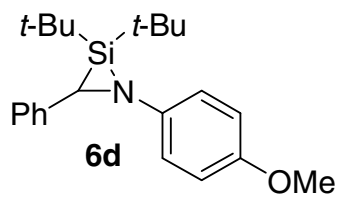

Silaaziridine 6d. The representative procedure for the synthesis of silaaziridines (NMR Experiment) was followed using AgOTf (0.3 mg, $1 \mathrm{~mol} \%)$, imine $5 \mathbf{d}\left(0.25 \mathrm{~mL}, 0.46 \mathrm{M}\right.$ in $\left.\mathrm{C}_{7} \mathrm{D}_{8}, 0.12 \mathrm{mmol}\right)$, silacyclopropane 2 (0.25 mL, $0.46 \mathrm{M}$ in $\left.\mathrm{C}_{7} \mathrm{D}_{8}, 0.12 \mathrm{mmol}\right)$, and $\mathrm{PhSiMe}_{3}(0.25 \mathrm{~mL}$ from silacyclopropane 2 stock solution, 0.092 M, $0.023 \mathrm{mmol})$. After $1 \mathrm{~h}$, silaaziridine $\mathbf{6 d}$ was formed in $76 \%$ yield as determined by ${ }^{1} \mathrm{H}$ NMR spectroscopy: ${ }^{1} \mathrm{H}$ NMR $\left(\mathrm{C}_{7} \mathrm{D}_{8}, 400 \mathrm{MHz}\right.$, distinctive peaks) $\delta 3.78(\mathrm{~s}, 1 \mathrm{H}), 3.41(\mathrm{~s}, 3 \mathrm{H}), 0.99(\mathrm{~s}, 9 \mathrm{H}), 0.95(\mathrm{~s}, 9 \mathrm{H}) ;{ }^{29} \mathrm{Si} \mathrm{NMR}$ $\left(\mathrm{C}_{7} \mathrm{D}_{8}, 99.3 \mathrm{MHz}\right) \delta-46.6$.

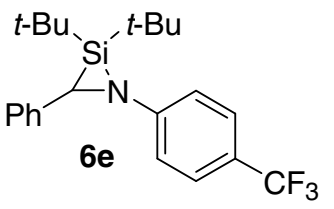

Silaaziridine 6e. The representative procedure for the synthesis of silaaziridines (NMR scale) was followed using AgOTf (0.3 mg, $1 \mathrm{~mol} \%)$, imine 5e $\left(0.25 \mathrm{~mL}, 0.46 \mathrm{M}\right.$ in $\left.\mathrm{C}_{7} \mathrm{D}_{8}, 0.12 \mathrm{mmol}\right)$, silacyclopropane $2(0.50 \mathrm{~mL}$, $0.46 \mathrm{M}$ in $\left.\mathrm{C}_{6} \mathrm{D}_{6}, 0.23 \mathrm{mmol}\right)$, and $\mathrm{PhSiMe}_{3}(0.25 \mathrm{~mL}$ from silacyclopropane 2 stock solution, $0.092 \mathrm{M}, 0.023$ mmol). After $1 \mathrm{~h}$, silaaziridine 6e was formed in $70 \%$ yield by ${ }^{1} \mathrm{H}$ NMR spectroscopy: ${ }^{1} \mathrm{H}$ NMR $\left(\mathrm{C}_{7} \mathrm{D}_{8}, 400\right.$ $\mathrm{MHz}$, distinctive peaks) $\delta 3.70(\mathrm{~s}, 1 \mathrm{H}), 0.90(\mathrm{~s}, 9 \mathrm{H}), 0.89(\mathrm{~s}, 9 \mathrm{H}) ;{ }^{29} \mathrm{Si} \mathrm{NMR}\left(\mathrm{C}_{7} \mathrm{D}_{8}, 99.3 \mathrm{MHz}\right) \delta-44.2$.

\section{Isolation of Silaaziridines 3, 6a, \& $6 c$}

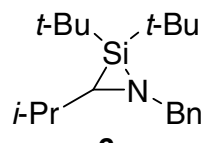

6 a

Representative Procedure for Metal-Catalyzed Silylene Transfer to Imines 5a, 1, and 5c: Synthesis of Silaaziridines 6a, 3, 6c.

Silaaziridine 6a. To a round-bottom flask were added imine 5a (2.13 g, $13.2 \mathrm{mmol})$, silacyclopropane 2 (2.96 $\mathrm{g}, 13.2 \mathrm{mmol})$, and toluene $(57 \mathrm{~mL}, 0.23 \mathrm{M})$. To the reaction mixture was added AgOTf $(0.34 \mathrm{~g}, 10 \mathrm{~mol} \%)$ to give a dark brown solution. The reaction mixture was stirred at $23{ }^{\circ} \mathrm{C}$ and was monitored by ${ }^{1} \mathrm{H}$ NMR spectroscopy. After $24 \mathrm{~h}$, the reaction mixture was concentrated in vacuo. Purification by bulb-to-bulb distillation (90-100 $\left.{ }^{\circ} \mathrm{C} / 0.05 \mathrm{~mm} \mathrm{Hg}\right)$ gave silaaziridine $6 \mathbf{a}$ as a clear liquid $(3.36 \mathrm{~g}, 84 \%):{ }^{1} \mathrm{H} \mathrm{NMR}\left(\mathrm{C}_{6} \mathrm{D}_{6}, 500\right.$ $\mathrm{MHz}) \delta 7.45(\mathrm{~m}, 2 \mathrm{H}), 7.22(\mathrm{~m}, 2 \mathrm{H}), 7.11(\mathrm{~m}, 1 \mathrm{H}), 4.46(\mathrm{~d}, J=13.6,1 \mathrm{H}), 4.05(\mathrm{~d}, J=13.6,1 \mathrm{H}), 1.97(\mathrm{~m}, 1 \mathrm{H})$,

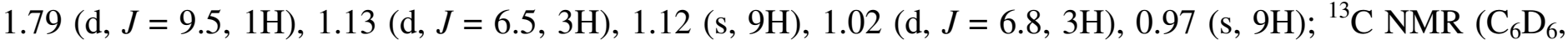


$125 \mathrm{MHz}) \delta 144.4,128.7,127.1,59.0,55.7,33.9,30.2,30.1,24.3,23.5,22.2,21.4 ;{ }^{29} \mathrm{Si} \mathrm{NMR}\left(\mathrm{C}_{7} \mathrm{D}_{8}, 99.3\right.$ MHz) $\delta$-46.5; IR (thin film) 3086, 2951, 1603, 1473, 1388, 1364, $\mathrm{cm}^{-1}$; HRMS (APCI) $\mathrm{m} / z$ calcd for $\mathrm{C}_{19} \mathrm{H}_{34} \mathrm{NSi}(\mathrm{M}+\mathrm{H})^{+}$304.2461, found 304.2459. Anal. Calcd for $\mathrm{C}_{19} \mathrm{H}_{33} \mathrm{NSi}$ : C, 75.18; H, 10.96; N, 4.61.

Found: C, 75.13; H, 11.07; N, 4.47.

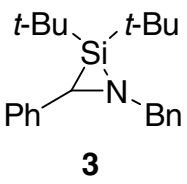

Silaaziridine 3. The representative procedure for the synthesis of silaaziridines was followed using imine 1 (0.59 g, $3.0 \mathrm{mmol})$, silacyclopropane 2 (0.67 g, $3.0 \mathrm{mmol})$, AgOTf (77 mg, $10 \mathrm{~mol} \%)$, and toluene (13 mL, 0.23 $\mathrm{M})$. After $24 \mathrm{~h}$, the reaction mixture was concentrated in vacuo. Purification by bulb-to-bulb distillation (106$\left.110{ }^{\circ} \mathrm{C} / 0.05 \mathrm{~mm} \mathrm{Hg}\right)$ gave silaaziridine 3 as a bright yellow oil $(0.81 \mathrm{~g}, 80 \%):{ }^{1} \mathrm{H}$ NMR $\left(\mathrm{C}_{7} \mathrm{D}_{8}, 500 \mathrm{MHz}\right) \delta$ 7.47-7.38 (m, 4H), 7.23-7.18 (m, 4H), $7.09(\mathrm{~m}, 1 \mathrm{H}), 7.01(\mathrm{~m}, 1 \mathrm{H}), 4.54(\mathrm{~d}, J=13.0,1 \mathrm{H}), 3.92(\mathrm{~d}, J=13.0$, $1 \mathrm{H}), 3.40(\mathrm{~s}, 1 \mathrm{H}), 0.99(\mathrm{~s}, 9 \mathrm{H}), 0.89(\mathrm{~s}, 9 \mathrm{H}) ;{ }^{13} \mathrm{C} \mathrm{NMR}\left(\mathrm{C}_{7} \mathrm{D}_{8}, 125 \mathrm{MHz}\right) \delta 145.1,142.9,128.5,128.4,128.1$, 127.0, 126.1, 124.3, 56.4, 47.5, 29.44, 29.35, 22.5, 22.3; ${ }^{29} \mathrm{Si} \mathrm{NMR}\left(\mathrm{C}_{7} \mathrm{D}_{8}, 99.3 \mathrm{MHz}\right) \delta-46.4$; IR (thin film) 3059, 2967, 1599, 1472, 1177, $\mathrm{cm}^{-1}$; HRMS (APCI) $\mathrm{m} / \mathrm{z}$ calcd for $\mathrm{C}_{22} \mathrm{H}_{32} \mathrm{NSi}(\mathrm{M}+\mathrm{H})^{+} 338.2304$, found 338.2289. Anal. Calcd for $\mathrm{C}_{22} \mathrm{H}_{31} \mathrm{NSi}$ : C, 78.27; H, 9.26; N, 4.15. Found: C, 78.14; H, 8.87; N, 4.49.

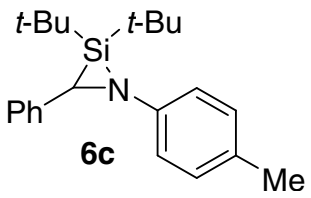

Silaaziridine 6c. The representative procedure for the synthesis of silaaziridines was followed using imine 5c (0.59 g, $3.0 \mathrm{mmol})$, silacyclopropane 2 (0.67 g, $3.0 \mathrm{mmol})$, AgOTf (77 mg, $10 \mathrm{~mol} \%)$, and toluene (13 mL, 0.23 $\mathrm{M})$. The reaction mixture was stirred for $24 \mathrm{~h}$, and purification by bulb-to-bulb distillation $\left(106-110{ }^{\circ} \mathrm{C} / 0.05\right.$ $\mathrm{mm} \mathrm{Hg}$ ) gave a bright yellow viscous oil. Further purification by trituration and crystallization from ether gave silaaziridine $6 \mathbf{c}$ as an analytically pure bright yellow solid $(0.74 \mathrm{~g}, 73 \%): \mathrm{mp} 65{ }^{\circ} \mathrm{C} ;{ }^{1} \mathrm{H} \mathrm{NMR}\left(\mathrm{C}_{6} \mathrm{D}_{6}, 400 \mathrm{MHz}\right)$ $\delta 7.38(\mathrm{~m}, 2 \mathrm{H}), 7.18(\mathrm{~m}, 2 \mathrm{H}), 7.03(\mathrm{~m}, 3 \mathrm{H}), 6.88(\mathrm{~m}, 2 \mathrm{H}), 3.86(\mathrm{~s}, 1 \mathrm{H}), 2.21(\mathrm{~s}, 3 \mathrm{H}), 0.97(\mathrm{~s}, 9 \mathrm{H}), 0.95(\mathrm{~s}, 9 \mathrm{H})$; ${ }^{13} \mathrm{C}$ NMR $\left(\mathrm{C}_{6} \mathrm{D}_{6}, 500 \mathrm{MHz}\right) \delta 148.4,143.3,130.6,128.7,127.8,126.7,125.2,116.8,43.7,29.5,29.3,22.9$, 22.7, 21.2; ${ }^{29} \mathrm{Si} \mathrm{NMR}\left(\mathrm{C}_{7} \mathrm{D}_{8}, 99.3 \mathrm{MHz}\right) \delta-46.3$; IR (thin film) 3020, 2933, 1612, 1508, $1472,1340 \mathrm{~cm}^{-1}$; HRMS (APCI) $m / z$ calcd for $\mathrm{C}_{22} \mathrm{H}_{32} \mathrm{NSi}(\mathrm{M}+\mathrm{H})^{+}$338.2304, found 338.2304. Anal. Calcd for $\mathrm{C}_{22} \mathrm{H}_{31} \mathrm{NSi}$ : C, 78.27; H, 9.26; N, 4.15. Found: C, 77.96; H, 9.20; N, 4.30 .

\section{Methanolysis of Silaaziridines $3 \&$ 6a-e}

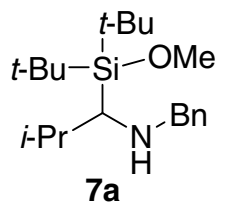

Representative Procedure for the Methanolysis of Silaaziridines 3, 6a-e: Synthesis of $\alpha-S i l y l$ Amines 7a7f.

$\boldsymbol{\alpha}$-Silyl Amine 7a. To a solution of $6 \mathbf{6}$ generated in situ $(1.0 \mathrm{mmol})$ in toluene $(5.0 \mathrm{~mL}, 0.20 \mathrm{M})$ was added $\mathrm{MeOH}(0.20 \mathrm{~mL}, 5.0 \mathrm{mmol})$. The reaction mixture was stirred at $23{ }^{\circ} \mathrm{C}$ for $1 \mathrm{~h}$, and filtered over a plug of silica gel with $15 \mathrm{~mL}$ of $\mathrm{CH}_{2} \mathrm{Cl}_{2}$. The filtrate was concentrated in vacuo. Analysis of the unpurified mixture by ${ }^{1} \mathrm{H}$ NMR spectroscopy showed a single regioisomer of product. Purification by flash chromatography (60:40 hexanes: $\left.\mathrm{CH}_{2} \mathrm{Cl}_{2}\right)$ gave $\alpha$-silyl amine $7 \mathbf{a}$ as a pale yellow liquid $(0.18 \mathrm{~g}, 53 \%):{ }^{1} \mathrm{H} \mathrm{NMR}\left(\mathrm{CDCl}_{3}, 500 \mathrm{MHz}\right) \delta$ $7.34(\mathrm{~m}, 2 \mathrm{H}), 7.30(\mathrm{~m}, 2 \mathrm{H}), 7.23(\mathrm{~m}, 1 \mathrm{H}), 4.04(\mathrm{~d}, J=12.5,1 \mathrm{H}), 3.68(\mathrm{~d}, J=12.5,1 \mathrm{H}), 3.62(\mathrm{~s}, 3 \mathrm{H}), 2.45(\mathrm{~d}, J$ $=4.1,1 \mathrm{H}), 2.17(\mathrm{septd}, J=6.9,4.1,1 \mathrm{H}), 1.16(\mathrm{~d}, J=6.9,3 \mathrm{H}), 1.12(\mathrm{~d}, J=6.9,3 \mathrm{H}), 1.11(\mathrm{~s}, 9 \mathrm{H}), 1.08(\mathrm{~s}, 9 \mathrm{H})$; ${ }^{13} \mathrm{C} \mathrm{NMR}\left(\mathrm{CDCl}_{3}, 125 \mathrm{MHz}\right) \delta 141.7,128.5,128.3,127.0,57.4,54.4,52.8,30.2,29.6,29.2,25.2,22.3,22.0$, 
19.7; IR (thin film) 3354, 3028, 2946, 1468, $1103 \mathrm{~cm}^{-1}$; HRMS (ES) $\mathrm{m} / z$ calcd for $\mathrm{C}_{20} \mathrm{H}_{38} \mathrm{NOSi}(\mathrm{M}+\mathrm{H})^{+}$ 336.2723, found 336.2721. Anal. Calcd for $\mathrm{C}_{20} \mathrm{H}_{37} \mathrm{NOSi}$ : C, 71.58; H, 11.11. Found: C, 71.76; H, 11.25.

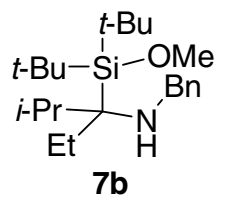

$\boldsymbol{\alpha}$-Silyl Amine 7b. The representative procedure for the synthesis of $\alpha$-silyl amines was followed using silaaziridine $\mathbf{6 b}$ generated in situ $(1.0 \mathrm{mmol})$, toluene $(5.0 \mathrm{~mL}, 0.20 \mathrm{M})$, and $\mathrm{MeOH}(0.20 \mathrm{~mL}, 5.0 \mathrm{mmol})$. Analysis of the unpurified mixture by ${ }^{1} \mathrm{H}$ NMR spectroscopy showed a single regioisomer of product. Purification by flash chromatography (99:1 hexanes: $\left.\mathrm{Et}_{3} \mathrm{~N}\right)$ gave $\alpha$-silyl amine $\mathbf{7 b}$ as a pale yellow liquid $(0.20$ $\mathrm{g}, 55 \%):{ }^{1} \mathrm{H}$ NMR $\left(\mathrm{CDCl}_{3}, 500 \mathrm{MHz}\right) \delta 7.35(\mathrm{~m}, 2 \mathrm{H}), 7.29(\mathrm{~m}, 2 \mathrm{H}), 7.22(\mathrm{~m}, 1 \mathrm{H}), 3.91(\mathrm{~d}, J=11.9,1 \mathrm{H}), 3.88$ (d, $J=11.8,1 \mathrm{H}), 3.64(\mathrm{~s}, 3 \mathrm{H}), 2.26$ (sept, $J=6.8,1 \mathrm{H}), 2.08(\mathrm{sext}, J=7.4,1 \mathrm{H}), 1.98(\mathrm{sext}, J=7.4,1 \mathrm{H}), 1.40$ (br, 1H) $1.18(\mathrm{~s}, 9 \mathrm{H}), 1.120(\mathrm{~s}, 9 \mathrm{H}), 1.124(\mathrm{~d}, J=6.8,3 \mathrm{H}), 1.11(\mathrm{~d}, J=6.8,3 \mathrm{H}), 1.04(\mathrm{t}, J=7.5,3 \mathrm{H}) ;{ }^{13} \mathrm{C} \mathrm{NMR}$ $\left(\mathrm{CDCl}_{3}, 125 \mathrm{MHz}\right) \delta 142.5,128.43,128.41,126.7,59.4,53.9,49.3,33.6,31.5,31.3,24.5,24.2,24.1,20.3$, 20.2, 11.9; IR (thin film) 3375, 3021, 2957, 1465, $1119 \mathrm{~cm}^{-1}$; HRMS (ES) $\mathrm{m} / z$ calcd for $\mathrm{C}_{22} \mathrm{H}_{42} \mathrm{NOSi}(\mathrm{M}+\mathrm{H})^{+}$ 364.3036, found 364.3023. Anal. Calcd for $\mathrm{C}_{22} \mathrm{H}_{41} \mathrm{NOSi}$ : C, 72.66; H, 11.36. Found: C, 72.40; H, 11.35.

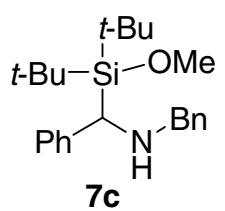

a-Silyl Amine 7c. The representative procedure for the synthesis of $\alpha$-silyl amines was followed using silaaziridine 3 generated in situ $(1.0 \mathrm{mmol})$, toluene $(5.0 \mathrm{~mL}, 0.20 \mathrm{M})$, and $\mathrm{MeOH}(0.20 \mathrm{~mL}, 5.0 \mathrm{mmol})$. Analysis of the unpurified mixture by ${ }^{1} \mathrm{H}$ NMR spectroscopy showed a single regioisomer of product. Purification by flash chromatography $\left(69: 30: 1\right.$ hexane $\left./ \mathrm{CH}_{2} \mathrm{Cl}_{2} / \mathrm{Et}_{3} \mathrm{~N}\right)$ gave $\alpha$-silyl amine 7c as a clear liquid $(0.23 \mathrm{~g}, 62 \%):{ }^{1} \mathrm{H} \mathrm{NMR}\left(\mathrm{CDCl}_{3}, 400 \mathrm{MHz}\right) \delta 7.39(\mathrm{~m}, 2 \mathrm{H}), 7.31-7.23(\mathrm{~m}, 7 \mathrm{H}), 7.14(\mathrm{~m}, 1 \mathrm{H}), 3.83(\mathrm{~d}, J=12.9$, $1 \mathrm{H}), 3.77(\mathrm{~s}, 1 \mathrm{H}), 3.462(\mathrm{~d}, J=12.8,1 \mathrm{H}), 3.457(\mathrm{~s}, 3 \mathrm{H}), 1.76(\mathrm{br}, 1 \mathrm{H}), 1.05(\mathrm{~s}, 9 \mathrm{H}), 0.97(\mathrm{~s}, 9 \mathrm{H}) ;{ }^{13} \mathrm{C} \mathrm{NMR}$ $\left(\mathrm{CDCl}_{3}, 125 \mathrm{MHz}\right) \delta 143.0,141.0,128.6,128.4,128.3,127.7,127.0,125.4,54.2,53.6,53.0,29.2,28.7,22.7$, 22.3; IR (thin film) 3083, 2936, 1599, 1474, $1108 \mathrm{~cm}^{-1}$; HRMS (ES) $\mathrm{m} / z$ calcd for $\mathrm{C}_{23} \mathrm{H}_{36} \mathrm{NOSi}(\mathrm{M}+\mathrm{H})^{+}$ 370.2566, found 370.2555. Anal. Calcd for $\mathrm{C}_{23} \mathrm{H}_{35} \mathrm{NOSi}$ : C, 74.74; H, 9.54; N, 3.79. Found: C, 74.71; H, 9.59; N, 3.73 .

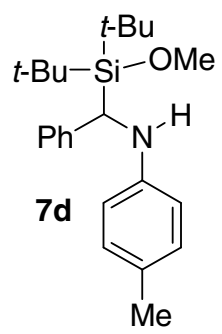

$\boldsymbol{\alpha}$-Silyl Amine 7d. The representative procedure for the synthesis of $\alpha$-silyl amines was followed using silaaziridine $6 \mathbf{c}(1.0 \mathrm{mmol})$, toluene $(5.0 \mathrm{~mL}, 0.20 \mathrm{M})$, and $\mathrm{MeOH}(0.20 \mathrm{~mL}, 5.0 \mathrm{mmol})$. Analysis of the unpurified mixture by ${ }^{1} \mathrm{H}$ NMR spectroscopy showed a single regioisomer of product. Purification by flash chromatography (98:2 hexane: $\left.\mathrm{CH}_{2} \mathrm{Cl}_{2}\right)$ gave $\alpha$-silyl amine $\mathbf{7 d}$ as a light orange liquid $(0.15 \mathrm{~g}, 40 \%)$ : ${ }^{1} \mathrm{H}$ NMR $\left(\mathrm{CDCl}_{3}, 500 \mathrm{MHz}\right) \delta 7.35(\mathrm{~m}, 2 \mathrm{H}), 7.23(\mathrm{~m}, 2 \mathrm{H}), 7.09(\mathrm{~m}, 1 \mathrm{H}), 6.88(\mathrm{~m}, 2 \mathrm{H}), 6.43(\mathrm{~m}, 2 \mathrm{H}), 4.41(\mathrm{~d}, J=7.0$, $1 \mathrm{H}), 4.34(\mathrm{~d}, J=7.0,1 \mathrm{H}), 3.67(\mathrm{~s}, 3 \mathrm{H}), 2.17(\mathrm{~s}, 3 \mathrm{H}), 1.04(\mathrm{~s}, 9 \mathrm{H}) 0.99(\mathrm{~s}, 9 \mathrm{H}) ;{ }^{13} \mathrm{C} \mathrm{NMR}\left(\mathrm{CDCl}_{3}, 125 \mathrm{MHz}\right) \delta$ 146.0, 143.1, 129.9, 128.4, 126.7, 126.3, 125.4, 113.1, 53.3, 48.6, 29.0, 28.7, 22.7, 22.5, 20.6.; IR (thin film) 3449, 3022, 2935, 1618, 1516, $1106 \mathrm{~cm}^{-1}$; HRMS (ES) $\mathrm{m} / z$ calcd for $\mathrm{C}_{23} \mathrm{H}_{36} \mathrm{NOSi}(\mathrm{M}+\mathrm{H})^{+} \quad 370.2566$, found 370.2569. Anal. Calcd for $\mathrm{C}_{23} \mathrm{H}_{35} \mathrm{NOSi}$ C, 74.74; H, 9.54. Found: C, 74.52; H, 9.79. 

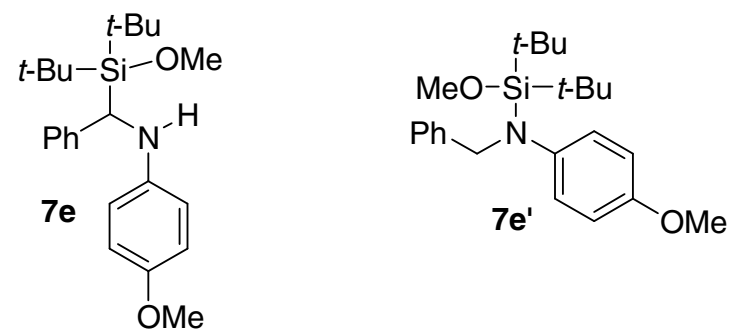

Silyl Amines 7e and 7e'. The representative procedure for the synthesis of $\alpha$-silyl amines was followed using silaaziridine $6 \mathbf{d}(1.0 \mathrm{mmol})$, toluene $(5.0 \mathrm{~mL}, 0.20 \mathrm{M})$, and $\mathrm{MeOH}(0.20 \mathrm{~mL}, 5.0 \mathrm{mmol})$. Analysis of the unpurified mixture by ${ }^{1} \mathrm{H}$ NMR spectroscopy showed a 13.2:1.0 mixture of regioisomeric products. Purification by flash chromatography (89:10:1 hexane: $\left.\mathrm{CH}_{2} \mathrm{Cl}_{2}: \mathrm{Et}_{3} \mathrm{~N}\right)$ gave $\alpha$-silyl amine 7e (major isomer) as a pale yellow liquid $(0.177 \mathrm{~g}, 46 \%): \quad{ }^{1} \mathrm{H}$ NMR $\left(\mathrm{C}_{7} \mathrm{D}_{8}, 500 \mathrm{MHz}\right) \delta 7.42(\mathrm{~m}, 2 \mathrm{H}), 7.15(\mathrm{~m}, 2 \mathrm{H}), 7.00(\mathrm{~m}, 1 \mathrm{H}), 6.61(\mathrm{~m}, 2 \mathrm{H})$, $6.45(\mathrm{~m}, 2 \mathrm{H}), 4.40(\mathrm{~d}, J=7.1,1 \mathrm{H}), 4.23(\mathrm{~d}, J=6.9,1 \mathrm{H}), 3.43(\mathrm{~s}, 3 \mathrm{H}), 3.32(\mathrm{~s}, 3 \mathrm{H}), 1.07(\mathrm{~s}, 9 \mathrm{H}), 0.99(\mathrm{~s}, 9 \mathrm{H})$; ${ }^{13} \mathrm{C}$ NMR $\left(\mathrm{C}_{7} \mathrm{D}_{8}, 125 \mathrm{MHz}\right) \delta 152.8,143.5,142.5,128.5,126.9,125.7,115.3,114.3,55.1,52.9,49.6,29.0$, 28.7, 22.6, 22.4; IR (thin film) 3444, 2935, 1509, $1237 \mathrm{~cm}^{-1}$; HRMS (ES) $m / z$ calcd for $\mathrm{C}_{23} \mathrm{H}_{36} \mathrm{NO}_{2} \mathrm{Si}(\mathrm{M}+\mathrm{H})^{+}$ 386.2515, found 386.2515. Anal. Calcd for $\mathrm{C}_{23} \mathrm{H}_{35} \mathrm{NO}_{2} \mathrm{Si}: \mathrm{C}, 71.64 ; \mathrm{H}, 9.15 ; \mathrm{N}, 3.63$. Found: C, 71.35; $\mathrm{H}$, 9.20, 3.65. Silyl amine 7e' (minor isomer) was isolated as a pale yellow liquid $(15 \mathrm{mg}, 4 \%)$ : ${ }^{1} \mathrm{H} \mathrm{NMR}\left(\mathrm{CDCl}_{3}\right.$, $400 \mathrm{MHz}) \delta 7.24(\mathrm{~m}, 2 \mathrm{H}), 7.18(\mathrm{~m}, 2 \mathrm{H}), 7.10-7.05(\mathrm{~m}, 3 \mathrm{H}), 6.67(\mathrm{~m}, 2 \mathrm{H}), 4.66(\mathrm{~s}, 2 \mathrm{H}), 3.69(\mathrm{~s}, 3 \mathrm{H}), 3.34(\mathrm{~s}$, $3 \mathrm{H}), 1.13(\mathrm{~s}, 18 \mathrm{H}) ;{ }^{13} \mathrm{C} \mathrm{NMR}\left(\mathrm{CDCl}_{3}, 125 \mathrm{MHz}\right) \delta 154.6,141.0,140.3,128.2,128.0,126.4,126.1,113.8,55.5$, 52.6, 52.1, 29.2, 23.2; HRMS (ES) $\mathrm{m} / z$ calcd for $\mathrm{C}_{23} \mathrm{H}_{35} \mathrm{NO}_{2} \mathrm{Si}(\mathrm{M}+\mathrm{Na})^{+}$408.2335, found 408.2349. Anal. Calcd for $\mathrm{C}_{23} \mathrm{H}_{35} \mathrm{NO}_{2} \mathrm{Si}$ : C, 71.64; H, 9.15. Found: C, 72.04; H, 9.44.

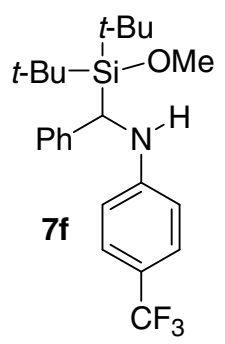

$\boldsymbol{\alpha}$-Silyl Amine 7f. The representative procedure for the synthesis of $\alpha$-silyl amines was followed using silaaziridine 6e $(1.0 \mathrm{mmol})$, toluene $(5.0 \mathrm{~mL}, 0.20 \mathrm{M})$, and $\mathrm{MeOH}(0.20 \mathrm{~mL}, 5.0 \mathrm{mmol})$. Analysis of the unpurified mixture by ${ }^{1} \mathrm{H}$ NMR spectroscopy showed a single regioisomer of product. Purification by successive flash chromatography (89:10:1 hexane: $\left.\mathrm{CH}_{2} \mathrm{Cl}_{2}: \mathrm{Et}_{3} \mathrm{~N}\right)$ gave $\alpha$-silyl amine $7 \mathbf{f}$ as a pale yellow liquid $(0.18 \mathrm{~g}, 43 \%)$. ${ }^{1} \mathrm{H}$ NMR $\left(\mathrm{CDCl}_{3}, 500 \mathrm{MHz}\right) \delta 7.33(\mathrm{~m}, 4 \mathrm{H}), 7.27(\mathrm{~m}, 2 \mathrm{H}), 7.15(\mathrm{~m}, 1 \mathrm{H}), 6.55(\mathrm{~d}, 2 \mathrm{H}), 4.95(\mathrm{br}$, $1 \mathrm{H}), 4.35$ (br, 1H), 3.72 (s, 3H), 1.04 (s, 9H) $1.03(\mathrm{~s}, 9 \mathrm{H}) ;{ }^{13} \mathrm{C} \mathrm{NMR}\left(\mathrm{CDCl}_{3}, 125 \mathrm{MHz}\right) \delta$ 150.7, 142.1, 128.6, $126.8(\mathrm{q}, J=4), 126.7,125.9,125.2\left(\mathrm{q},{ }^{1} J_{\mathrm{CF}}=269\right), 118.9\left(\mathrm{q},{ }^{2} J_{\mathrm{CF}}=31\right), 112.4,53.5,48.2,29.0,28.6,22.8$, $22.5 ;{ }^{13} \mathrm{C}$ NMR (DMSO, $\left.125 \mathrm{MHz}\right) \delta 151.5,142.2,128.0,126.4,126.0(\mathrm{q}, J=4), 125.3,125.2\left(\mathrm{q},{ }^{1} J_{\mathrm{CF}}=268\right)$, $116.0\left(\mathrm{q},{ }^{2} J_{\mathrm{CF}}=33\right), 112.4,52.7,46.3,28.4,28.2,21.8,21.7$; HRMS (ES) $\mathrm{m} / z$ calcd for $\mathrm{C}_{23} \mathrm{H}_{33} \mathrm{~F}_{3} \mathrm{NOSi}(\mathrm{M}+$ $\mathrm{H})^{+}$424.2284, found 424.2288. Anal. Calcd for $\mathrm{C}_{23} \mathrm{H}_{32} \mathrm{~F}_{3} \mathrm{NOSi}$ : C, 65.22; H, 7.61. Found: C, 65.43; H, 7.86.

\section{AgOTf-Catalyzed Silylene Transfer to Conjugated Imines 8 \& 11}

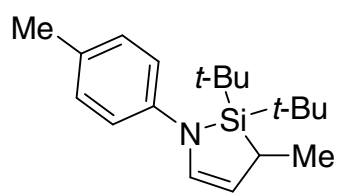

9

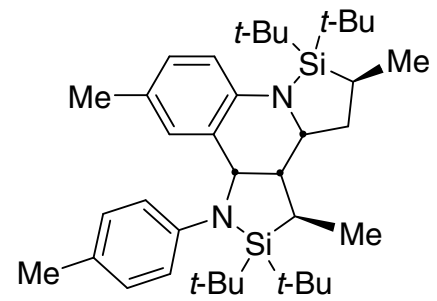

10

Enamine 9 (NMR Experiment). To an NMR tube were added AgOTf $(0.3 \mathrm{mg}, 1 \mathrm{~mol} \%)$, imine 8 (0.25 mL, $0.46 \mathrm{M}$ in $\left.\mathrm{C}_{7} \mathrm{D}_{8}, 0.12 \mathrm{mmol}\right)$, silacyclopropane $2\left(0.25 \mathrm{~mL}, 0.46 \mathrm{M}\right.$ in $\left.\mathrm{C}_{7} \mathrm{D}_{8}, 0.12 \mathrm{mmol}\right)$, and $\mathrm{PhSiMe}_{3}(0.25 \mathrm{~mL}$ 
from silacyclopropane 2 stock solution, $0.092 \mathrm{M}, 0.023 \mathrm{mmol}$ ). The reaction mixture was left at $23{ }^{\circ} \mathrm{C}$ and monitored by ${ }^{1} \mathrm{H}$ NMR spectroscopy. After $3 \mathrm{~h}$, enamine 9 was formed in $80 \%$ yield as determined by ${ }^{1} \mathrm{H}$ NMR spectroscopy: ${ }^{1} \mathrm{H}$ NMR $\left(\mathrm{C}_{7} \mathrm{D}_{8}, 400 \mathrm{MHz}\right.$, distinctive peaks) $\delta 6.38(\mathrm{dd}, J=5.3,2.7,1 \mathrm{H}), 4.85(\mathrm{dd}, J=5.3,2.7$, $1 \mathrm{H}), 2.12(\mathrm{~s}, 3 \mathrm{H}), 1.32(\mathrm{~d}, J=7.7,3 \mathrm{H}), 1.13(\mathrm{~s}, 9 \mathrm{H}), 1.01(\mathrm{~s}, 9 \mathrm{H})$. The reaction mixture was extracted with hexanes $(4 \mathrm{~mL})$ and $\mathrm{CH}_{3} \mathrm{CN}(3 \times 4 \mathrm{~mL})$ and the hexane layer was concentrated in vacuo. Unpurified enamine 9 was characterized on the basis of ${ }^{1} \mathrm{H}$ and ${ }^{13} \mathrm{C}$ NMR spectral data, IR, and mass spectrometry data: ${ }^{1} \mathrm{H}$ NMR $\left(\mathrm{C}_{7} \mathrm{D}_{8}, 500 \mathrm{MHz}\right) \delta 6.95(\mathrm{~m}, 2 \mathrm{H}), 6.89(\mathrm{~m}, 2 \mathrm{H}), 6.39(\mathrm{dd}, J=5.3,2.7,1 \mathrm{H}), 4.86(\mathrm{dd}, J=5.3,2.7,1 \mathrm{H}), 2.16(\mathrm{qd}$, $J=7.7,2.7,1 \mathrm{H}), 2.12(\mathrm{~s}, 3 \mathrm{H}), 1.32(\mathrm{~d}, J=7.7,3 \mathrm{H}), 1.13(\mathrm{~s}, 9 \mathrm{H}), 1.01(\mathrm{~s}, 9 \mathrm{H}) ;{ }^{13} \mathrm{C} \mathrm{NMR}\left(\mathrm{C}_{7} \mathrm{D}_{8}, 125 \mathrm{MHz}\right) \delta$ $145.2,138.7,130.3,129.9,120.8,111.3,28.9,28.4,26.8,23.8,22.7,21.4,16.7$; IR (thin film) 3026, 2933, $1611,1510,1288 \mathrm{~cm}^{-1}$; HRMS (ES) $\mathrm{m} / z$ calcd for $\mathrm{C}_{19} \mathrm{H}_{32} \mathrm{NSi}(\mathrm{M}+\mathrm{H})^{+} \quad 302.2304$, found 302.2305.

Dimer 10. To a round-bottom flask were added imine $8(0.16 \mathrm{~g}, 1.0 \mathrm{mmol})$, silacyclopropane $2(0.22 \mathrm{~g}, 1.0$ $\mathrm{mmol})$, and toluene $(5 \mathrm{~mL}, 0.20 \mathrm{M})$. To the reaction mixture was added AgOTf $(2.6 \mathrm{mg}, 1 \mathrm{~mol} \%)$ to give a dark brown solution. The reaction mixture was stirred at $23{ }^{\circ} \mathrm{C}$ for $1 \mathrm{~h}$. Concentration of the reaction mixture without prior hexanes: $\mathrm{CH}_{3} \mathrm{CN}$ extraction (see previous procedure) resulted in dimerization of enamine 9 to give compound $\mathbf{1 0}$ as a single diastereomer on the basis of ${ }^{1} \mathrm{H}$ NMR spectroscopy. Purification of $\mathbf{1 0}$ by flash chromatography (100\% hexanes) yielded the product as a white solid $(0.24 \mathrm{~g}, 40 \%)$. X-ray quality crystals were grown from ether by slow evaporation: $\mathrm{mp} 223{ }^{\circ} \mathrm{C} ;{ }^{1} \mathrm{H} \mathrm{NMR}\left(\mathrm{CDCl}_{3}, 400 \mathrm{MHz}\right) \delta 7.03(\mathrm{~b}, 4 \mathrm{H}), 6.94(\mathrm{~b}$, $1 \mathrm{H}), 6.69(\mathrm{~m}, 1 \mathrm{H}), 6.64(\mathrm{~m}, 1 \mathrm{H}), 5.39(\mathrm{~d}, J=7.2,1 \mathrm{H}), 4.07(\mathrm{bd}, J=10.8,1 \mathrm{H}), 2.32-2.28$ (m-overlapped, 4H), $2.20(\mathrm{~m}, 1 \mathrm{H}), 2.02(\mathrm{~m}, 1 \mathrm{H}), 1.98(\mathrm{~s}, 3 \mathrm{H}), 1.65-1.55(\mathrm{~m}, 2 \mathrm{H}), 1.45(\mathrm{~d}, J=7.4,3 \mathrm{H}), 1.29(\mathrm{~d}, J=7.2,3 \mathrm{H}), 1.23(\mathrm{~s}$, 9H), $1.14(\mathrm{~s}, 9 \mathrm{H}), 0.90(\mathrm{~s}, 9 \mathrm{H}), 0.82(\mathrm{~s}, 9 \mathrm{H}) ;{ }^{13} \mathrm{C} \mathrm{NMR}\left(\mathrm{CDCl}_{3}, 125 \mathrm{MHz}\right) \delta 149.0,142.2,130.9,129.6,129.1$, 126.6, 126.0, 117.4, 65.2, 60.4, 48.0, 37.3, 29.7, 29.63, 29.59, 29.4, 25.1, 24.0, 22.3, 22.0, 20.9, 20.7, 17.5, 17.0, 16.9, 15.1; IR (thin film) 3083, 2965, 1612, 1498, $1274 \mathrm{~cm}^{-1}$; HRMS (ES) $m / z$ calcd for $\mathrm{C}_{38} \mathrm{H}_{63} \mathrm{~N}_{2} \mathrm{Si}_{2}(\mathrm{M}+\mathrm{H})^{+}$ 603.4530, found 603.4521. Anal. Calcd for $\mathrm{C}_{38} \mathrm{H}_{62} \mathrm{~N}_{2} \mathrm{Si}_{2}$ : C, 75.68; H, 10.36; N, 4.65. Found: C, 75.64; H, $10.51 ; \mathrm{N}, 4.47$.

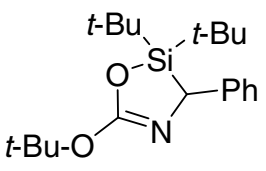

12

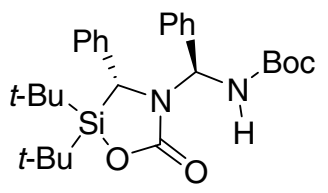

13

Oxazoline 12 (NMR Experiment). To an NMR tube were added AgOTf (3 mg, $10 \mathrm{~mol} \%)$, imine 11 (0.25 mL, $0.46 \mathrm{M}$ in $\left.\mathrm{C}_{7} \mathrm{D}_{8}, 0.12 \mathrm{mmol}\right)$, silacyclopropane $2\left(0.25 \mathrm{~mL}, 0.46 \mathrm{M}\right.$ in $\left.\mathrm{C}_{7} \mathrm{D}_{8}, 0.12 \mathrm{mmol}\right)$, and $\mathrm{PhSiMe}_{3}(0.25 \mathrm{~mL}$ from silacyclopropane 2 stock solution, $0.092 \mathrm{M}, 0.023 \mathrm{mmol}$ ). The reaction mixture was left at $23{ }^{\circ} \mathrm{C}$ and monitored by ${ }^{1} \mathrm{H}$ NMR spectroscopy. After $3 \mathrm{~h}$, further production of product appeared to cease, with a remaining amount of imine $\mathbf{1 1}$ and silacyclopropane $\mathbf{2}$. The oxazoline $\mathbf{1 2}$ was formed in $46 \%$ yield as determined by ${ }^{1} \mathrm{H}$ NMR spectroscopy: ${ }^{1} \mathrm{H}$ NMR $\left(\mathrm{C}_{7} \mathrm{D}_{8}, 500 \mathrm{MHz}\right.$, distinctive peaks) $\delta 4.73(\mathrm{~s}, 1 \mathrm{H}), 1.59$ (s, 9H), 1.05 (s, 9H), 0.76 (s, 9H). ${ }^{29} \mathrm{Si} \mathrm{NMR}\left(\mathrm{C}_{7} \mathrm{D}_{8}, 99.3 \mathrm{MHz}\right) \delta 25.6$.

Carbamic Acid Silyl Ester 13. To a round-bottom flask were added imine 11 (0.62 g, $3.0 \mathrm{mmol})$, silacyclopropane $2(0.67 \mathrm{~g}, 3.0 \mathrm{mmol})$, and toluene $(13 \mathrm{~mL}, 0.23 \mathrm{M})$. To the reaction mixture was added AgOTf (77 mg, $10 \mathrm{~mol} \%$ ) to give a dark brown solution. The reaction mixture was stirred at $25{ }^{\circ} \mathrm{C}$ for $3 \mathrm{~h}$. Concentration of the reaction mixture resulted in formation of compound $\mathbf{1 3}$ as a single diastereomer on the basis of ${ }^{1} \mathrm{H}$ NMR spectroscopy. Purification of $\mathbf{1 3}$ by trituration gave the product as a white solid $(0.60 \mathrm{~g}$, $40 \%)$. X-ray quality crystals were grown from $\mathrm{CH}_{2} \mathrm{Cl}_{2}$ by slow evaporation: $\mathrm{mp} 185{ }^{\circ} \mathrm{C} ;{ }^{1} \mathrm{H} \mathrm{NMR}\left(\mathrm{CDCl}_{3}, 500\right.$ MHz) $\delta 7.44(\mathrm{~m}, 3 \mathrm{H}), 7.36-7.23(\mathrm{~m}, 7 \mathrm{H}), 5.81(\mathrm{~d}, J=10.0,1 \mathrm{H}), 4.41(\mathrm{~s}, 1 \mathrm{H}), 1.46(\mathrm{~s}, 9 \mathrm{H}), 0.92(\mathrm{~s}, 9 \mathrm{H}), 0.78(\mathrm{~s}$, $9 \mathrm{H}) ;{ }^{13} \mathrm{C} \mathrm{NMR}\left(\mathrm{CDCl}_{3}, 125 \mathrm{MHz}\right) \delta 158.0,154.2,138.7,137.0,129.1,128.7,128.6,127.1,127.0,126.3,80.0$, 66.6, 51.6, 28.5, 27.4, 26.8, 21.5, 20.9.; IR (thin film) 3425, 3089, 2973, 1720, 1488, $1158 \mathrm{~cm}^{-1}$; HRMS (ES) $m / z$ calcd for $\mathrm{C}_{28} \mathrm{H}_{40} \mathrm{~N}_{2} \mathrm{O}_{4} \mathrm{SiNa}(\mathrm{M}+\mathrm{Na})^{+}$519.2655, found 519.2640. Anal. Calcd for $\mathrm{C}_{28} \mathrm{H}_{40} \mathrm{~N}_{2} \mathrm{O}_{4} \mathrm{Si}: \mathrm{C}, 67.70$; H, 8.12; N, 5.64. Found: C, 67.71; H, 8.22; N, 5.46. 


\section{Electrophile Insertions of Silaaziridine $6 \mathbf{a}$}
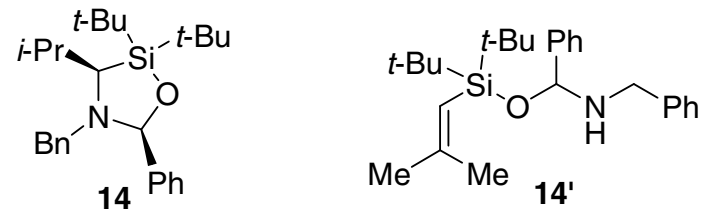

$\boldsymbol{N}, \boldsymbol{O}$-acetal 14 (NMR Experiment). To an NMR tube were added AgOTf $(0.3 \mathrm{mg}, 1 \mathrm{~mol} \%)$, silaaziridine 6a $\left(0.50 \mathrm{~mL}, 0.23 \mathrm{M}\right.$ in $\left.\mathrm{C}_{7} \mathrm{D}_{8}, 0.12 \mathrm{mmol}\right)$, benzaldehyde $(37 \mu \mathrm{L}, 0.36 \mathrm{mmol})$, and $\mathrm{PhSiMe}_{3}(0.50 \mathrm{~mL}$ from silaaziridine 6a stock solution, $0.046 \mathrm{M}, 0.023 \mathrm{mmol}$ ). The reaction mixture was left at $23{ }^{\circ} \mathrm{C}$ and monitored by ${ }^{1} \mathrm{H}$ NMR spectroscopy. After $30 \mathrm{~min}$, silaaziridine was consumed to give a $99 \%$ yield of $N, O$-acetals as a 75:25 cis:trans mixture. The mixture equilibrated to a $91: 9$ ratio after $8 \mathrm{~h}$ as determined by ${ }^{1} \mathrm{H}$ NMR spectroscopy: ${ }^{1} \mathrm{H}$ NMR $\left(\mathrm{C}_{7} \mathrm{D}_{8}, 400 \mathrm{MHz}\right.$, distinctive peaks for major diastereomer) $\delta 5.50(\mathrm{~s}, 1 \mathrm{H}), 4.01(\mathrm{~d}, J=14.1,1 \mathrm{H}), 3.80$ $(\mathrm{d}, J=14.1,1 \mathrm{H}), 2.35(\mathrm{~d}, J=10.4,1 \mathrm{H}), 2.24(\mathrm{septd}, J=6.5,10.4,1 \mathrm{H}), 1.27(\mathrm{~d}, J=6.4,3 \mathrm{H}), 1.10(\mathrm{~s}, 9 \mathrm{H}), 1.00$ $(\mathrm{s}, 9 \mathrm{H}), 0.93(\mathrm{~d}, J=6.5,3 \mathrm{H})$. The cis-stereochemistry of 14 was determined by NOE spectroscopy. In the absence of AgOTf, the reaction was much slower, and by-product formation was observed (after $24 \mathrm{~h}$ only $43 \%$ of product 14 was formed as a 65:35 cis:trans mixture, as determined by ${ }^{1} \mathrm{H}$ NMR spectroscopy).

Vinyl Silane 14'. To a round-bottom flask were added silaaziridine 6a $(0.74 \mathrm{~g}, 2.4 \mathrm{mmol})$, toluene $(10 \mathrm{~mL}$, $0.24 \mathrm{M})$, benzaldehyde $(0.73 \mathrm{~mL}, 7.2 \mathrm{mmol})$, and AgOTf $(62 \mathrm{mg}, 10 \mathrm{~mol} \%)$. The reaction mixture was stirred at $23{ }^{\circ} \mathrm{C}$ for $24 \mathrm{~h}$, then filtered over a plug of silica gel with $10 \mathrm{~mL}$ of $\mathrm{CH}_{2} \mathrm{Cl}_{2}$. The filtrate was concentrated in vacuo and purified by flash chromatography (99:1 hexanes: $\left.\mathrm{Et}_{3} \mathrm{~N}\right)$. A significant amount of decomposition, $\mathrm{N}, \mathrm{O}$-acetal hydrolysis and elimination product 14' were observed after purification. Vinyl silane 14' was isolated as a clear liquid $(0.13 \mathrm{~g}, 13 \%):{ }^{1} \mathrm{H} \mathrm{NMR}\left(\mathrm{CDCl}_{3}, 400 \mathrm{MHz}\right) \delta 7.32-7.16(\mathrm{~m}, 10 \mathrm{H}), 5.75(\mathrm{~m}, 1 \mathrm{H}), 4.92$ $(\mathrm{s}, 2 \mathrm{H}), 4.31(\mathrm{~s}, 2 \mathrm{H}), 1.48(\mathrm{~d}, J=1.0,3 \mathrm{H}), 1.47(\mathrm{~d}, J=1.0,3 \mathrm{H}), 1.16(\mathrm{~s}, 18 \mathrm{H}) ;{ }^{13} \mathrm{C} \mathrm{NMR}\left(\mathrm{CDCl}_{3}, 100 \mathrm{MHz}\right) \delta$ $141.8,141.6,129.4,128.3,128.0,127.8,126.9,126.3,125.8,122.1,65.8,52.7,29.2,23.8,23.3$, 18.5.; IR (thin film) 3412, 3087, 2933, 1659, 1473, $1113 \mathrm{~cm}^{-1}$; HRMS (ES) $\mathrm{m} / z$ calcd for $\mathrm{C}_{26} \mathrm{H}_{40} \mathrm{NOSi}(\mathrm{M}+\mathrm{H})^{+} \quad 410.2879$, found 410.2870. Anal. Calcd for $\mathrm{C}_{26} \mathrm{H}_{39} \mathrm{NOSi}$ : C, 76.23; H, 9.60; N, 3.42. Found: C, 76.16; H, 9.75; N, 3.54.
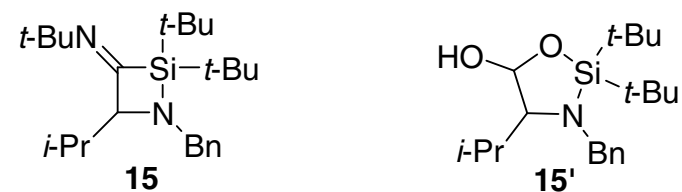

Iminosilacyclobutane 15 (NMR Experiment). The representative procedure for the synthesis of silaaziridines (NMR Experiment) was followed to generate silaaziridine 6a. After in situ generation of silaaziridine 6a, $t$ butyl isocyanide was added $(41 \mu \mathrm{L}, 0.36 \mathrm{mmol})$. The reaction mixture was left at $23{ }^{\circ} \mathrm{C}$ and monitored by ${ }^{1} \mathrm{H}$ NMR spectroscopy. After $12 \mathrm{~h}$, iminosilacyclobutane 15 was formed in 95\% yield as a single regioisomer as determined by ${ }^{1} \mathrm{H}$ NMR spectroscopy: ${ }^{1} \mathrm{H}$ NMR $\left(\mathrm{C}_{7} \mathrm{D}_{8}, 400 \mathrm{MHz}\right.$, distinctive peaks $) \delta 4.40(\mathrm{~d}, J=15.4,1 \mathrm{H})$, $3.93(\mathrm{~d}, J=15.5,1 \mathrm{H}), 3.86(\mathrm{~d}, J=2.5,1 \mathrm{H}), 1.22(\mathrm{~s}, 9 \mathrm{H}), 1.16(\mathrm{~s}, 9 \mathrm{H}), 1.09(\mathrm{~s}, 9 \mathrm{H})$.

Iminosilacyclobutane 15. To a round-bottom flask were added silaaziridine 6a $(0.79 \mathrm{~g}, 2.6 \mathrm{mmol})$, toluene (11 $\mathrm{mL}, 0.24 \mathrm{M})$, and $t$-butyl isocyanide $(0.88 \mathrm{~mL}, 7.8 \mathrm{mmol})$. The reaction mixture was stirred at $25^{\circ} \mathrm{C}$ for $19 \mathrm{~h}$ and concentrated in vacuo to provide a clear liquid $(0.90 \mathrm{~g}, 90 \%)$ that slowly solidified after several days. Purification by crystallization (ether) provided $\mathbf{1 5}$ as an analytically pure solid: $\mathrm{mp} 55-57{ }^{\circ} \mathrm{C}$; ${ }^{1} \mathrm{H}$ NMR $\left(\mathrm{CDCl}_{3}, 400 \mathrm{MHz}\right) \delta 7.42(\mathrm{~m}, 2 \mathrm{H}), 7.30(\mathrm{~m}, 2 \mathrm{H}), 7.20(\mathrm{~m}, 1 \mathrm{H}), 4.53(\mathrm{~d}, J=15.9,1 \mathrm{H}), 4.05(\mathrm{~d}, J=15.8,1 \mathrm{H})$, $3.77(\mathrm{~d}, J=2.5,1 \mathrm{H}), 1.82(\mathrm{septd}, J=7.0,2.5,1 \mathrm{H}), 1.22(\mathrm{~s}, 9 \mathrm{H}), 1.18(\mathrm{~s}, 9 \mathrm{H}), 1.14(\mathrm{~s}, 9 \mathrm{H}), 0.95(\mathrm{~d}, J=7.1,3 \mathrm{H})$, $0.75(\mathrm{~d}, J=7.0,3 \mathrm{H}) ;{ }^{13} \mathrm{C} \mathrm{NMR}\left(\mathrm{CDCl}_{3}, 125 \mathrm{MHz}\right) \delta 179.1,143.0,128.2,127.9,126.6,83.0,57.1,53.6,32.1$, 29.8, 29.6, 29.1, 24.49, 24.46, 20.0, 17.7; IR (thin film) 3086, 2968, 1654, $1473 \mathrm{~cm}^{-1}$; HRMS (ES) $\mathrm{m} / z$ calcd for $\mathrm{C}_{24} \mathrm{H}_{43} \mathrm{~N}_{2} \mathrm{Si}(\mathrm{M}+\mathrm{H})^{+}$387.3195, found 387.3191. Anal. Calcd for $\mathrm{C}_{24} \mathrm{H}_{42} \mathrm{~N}_{2} \mathrm{Si}$ : C, 74.54; H, 10.95; N, 7.24. Found: C, 74.42; H, 11.15; N, 7.01. Purification of 15 by flash chromatography $\left(90: 10\right.$ hexanes: $\left.\mathrm{CH}_{2} \mathrm{Cl}_{2}\right)$ resulted in hydrolysis to provide hemiacetal $\mathbf{1 5}^{\prime}$ as a single diastereomer.

Hemiacetal 15'. Hemiacetal 15' was isolated by flash chromatography (94:5:1 hexanes: $\left.\mathrm{CH}_{2} \mathrm{Cl}_{2}: \mathrm{Et}_{3} \mathrm{~N}\right)$ as a white solid (0.50 g, 55\%): $\mathrm{mp} 130-135{ }^{\circ} \mathrm{C} ;{ }^{1} \mathrm{H} \mathrm{NMR}\left(\mathrm{CDCl}_{3}, 400 \mathrm{MHz}\right) \delta 7.38(\mathrm{~m}, 2 \mathrm{H}), 7.30(\mathrm{~m}, 2 \mathrm{H}), 7.22$ 
$(\mathrm{m}, 1 \mathrm{H}), 5.30(\mathrm{dd}, J=5.0,5.01 \mathrm{H}), 4.36(\mathrm{~d}, J=16.0,1 \mathrm{H}), 3.90(\mathrm{~d}, J=16.0,1 \mathrm{H}), 3.27(\mathrm{~d}, J=5.0,1 \mathrm{H}), 3.00(\mathrm{dd}$, $J=5.2,3.4,1 \mathrm{H}), 1.75(\mathrm{septd}, J=7.0,3.4,1 \mathrm{H}), 1.12(\mathrm{~s}, 9 \mathrm{H}), 1.11(\mathrm{~s}, 9 \mathrm{H}), 0.84(\mathrm{~d}, J=7.2,3 \mathrm{H}), 0.78(\mathrm{~d}, J=6.8$, $3 \mathrm{H}) ;{ }^{13} \mathrm{C} \mathrm{NMR}\left(\mathrm{CDCl}_{3}, 125 \mathrm{MHz}\right) \delta 142.2,128.4,127.7,126.9,95.5,73.5,51.4,28.3,28.2 .27 .5,23.0,22.1$, 20.0, 16.2; IR (thin film) 3373, 3084, 2968, $1474 \mathrm{~cm}^{-1}$; HRMS (ES) $\mathrm{m} / z$ calcd for $\mathrm{C}_{20} \mathrm{H}_{35} \mathrm{NO}_{2} \mathrm{SiNa}(\mathrm{M}+\mathrm{Na})^{+}$ 372.2335, found 372.2334. Anal. Calcd for $\mathrm{C}_{20} \mathrm{H}_{35} \mathrm{NO}_{2} \mathrm{Si}: \mathrm{C}, 68.71 ; \mathrm{H}, 10.09 ; \mathrm{N}, 4.01$. Found: C, 68.41; H, $10.03 ; \mathrm{N}, 4.16$.

\section{Palladium-Catalyzed Synthesis of Azasilacyclopentenes 16a-f}
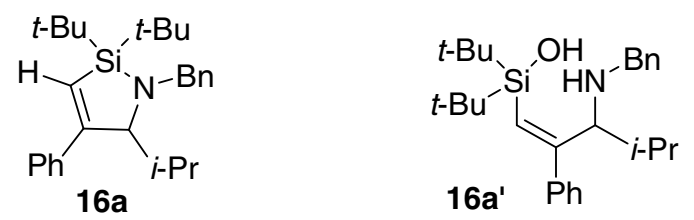

Azasilacyclopentene 16a (NMR Experiment). To an NMR tube were added silaaziridine 6a $(0.50 \mathrm{~mL}, 0.23$ $\mathrm{M}$ in $\left.\mathrm{C}_{7} \mathrm{D}_{8}, 0.12 \mathrm{mmol}\right)$, phenylacetylene $(15 \mu \mathrm{L}, 0.14 \mathrm{mmol}), \mathrm{PhSiMe}_{3}(0.50 \mathrm{~mL}$ from silaaziridine 6a stock solution, $0.046 \mathrm{M}, 0.023 \mathrm{mmol})$, and $\mathrm{Pd}\left(\mathrm{PPh}_{3}\right)_{4}(1 \mathrm{mg}, 1 \mathrm{~mol} \%)$. The bright yellow reaction mixture was placed in an oil bath at $80{ }^{\circ} \mathrm{C}$ and the reaction progress was monitored by ${ }^{1} \mathrm{H}$ NMR spectroscopy. After $8 \mathrm{~h}$, azasilacyclopentene 16a was formed in $92 \%$ yield as determined by ${ }^{1} \mathrm{H}$ NMR spectroscopy: ${ }^{1} \mathrm{H}$ NMR $\left(\mathrm{C}_{7} \mathrm{D}_{8}\right.$, $400 \mathrm{MHz}$, distinctive peaks) $\delta 5.87(\mathrm{~d}, J=1.4,1 \mathrm{H}), 4.39(\mathrm{~d}, J=15.7,1 \mathrm{H}), 4.37(\mathrm{bs}, 1 \mathrm{H}), 4.24(\mathrm{~d}, J=16.0,1 \mathrm{H})$, $1.20(\mathrm{~s}, 9 \mathrm{H}), 1.11(\mathrm{~s}, 9 \mathrm{H}), 0.87(\mathrm{~d}, J=7.0,3 \mathrm{H}), 0.48(\mathrm{~d}, J=7.3,3 \mathrm{H})$.

Azasilacyclopentene 16a. To a round-bottom flask were added silaaziridine $6 \mathbf{a}(0.37 \mathrm{~g}, 1.2 \mathrm{mmol})$, phenylacetylene $(0.16 \mathrm{~mL}, 1.5 \mathrm{mmol})$, toluene $(5 \mathrm{~mL}, 0.25 \mathrm{M})$, and $\mathrm{Pd}\left(\mathrm{PPh}_{3}\right)_{4}(14 \mathrm{mg}, 1 \mathrm{~mol} \%)$. The reaction mixture was heated in an oil bath at $80{ }^{\circ} \mathrm{C}$. After $8 \mathrm{~h}$, the reaction mixture was cooled, then extracted with hexanes $(10 \mathrm{~mL})$ and $\mathrm{CH}_{3} \mathrm{CN}(2 \mathrm{x} 10 \mathrm{~mL})$ to remove the palladium catalyst. The hexane layers were concentrated in vacuo to give $\mathbf{1 6 a}$ as a pale yellow oil $(0.45 \mathrm{~g}, 90 \%)$. Unpurified 16a was characterized on the basis of ${ }^{1} \mathrm{H}$ and ${ }^{13} \mathrm{C}$ NMR spectral data, IR, and mass spectrometry data: ${ }^{1} \mathrm{H}$ NMR $\left(\mathrm{CDCl}_{3}, 500 \mathrm{MHz}\right) \delta 7.45$ $(\mathrm{m}, 2 \mathrm{H}), 7.35(\mathrm{~m}, 2 \mathrm{H}), 7.30-7.17(\mathrm{~m}, 6 \mathrm{H}), 5.91(\mathrm{bs}, 1 \mathrm{H}), 4.44(\mathrm{~d}, J=16.2,1 \mathrm{H}), 4.32(\mathrm{bs}, 1 \mathrm{H}), 4.28(\mathrm{~d}, J=16.1$, $1 \mathrm{H}), 1.98$ (sept, $J=7.1,1 \mathrm{H}), 1.15(\mathrm{~s}, 9 \mathrm{H}), 1.06(\mathrm{~s}, 9 \mathrm{H}), 0.74(\mathrm{~d}, J=7.0,3 \mathrm{H}), 0.42(\mathrm{~d}, J=7.3,3 \mathrm{H}) ;{ }^{13} \mathrm{C} \mathrm{NMR}$ $\left(\mathrm{CDCl}_{3}, 125 \mathrm{MHz}\right) \delta 164.5,143.3,142.3,128.3,128.0,127.9,127.3,127.1,126.6,126.4,73.9,51.7,30.0,29.7$, 29.1, 23.7, 22.3, 21.7, 17.3; IR (thin film) 3083, 2930, 1472, $1091 \mathrm{~cm}^{-1}$; HRMS (ES) $\mathrm{m} / z$ calcd for $\mathrm{C}_{27} \mathrm{H}_{40} \mathrm{NSi}$ $(\mathrm{M}+\mathrm{H})^{+}$406.2930, found 406.2930. Silanol 16a'. Purification of 16a by flash chromatography (89:10:1 pentane: $\left.\mathrm{CH}_{2} \mathrm{Cl}_{2}: \mathrm{Et}_{3} \mathrm{~N}\right)$ resulted in hydrolysis of 16 a to provide 16a' as a white solid $(0.33 \mathrm{~g}, 76 \%)$ : $\mathrm{mp} 107^{\circ} \mathrm{C}$; ${ }^{1} \mathrm{H} \mathrm{NMR}\left(\mathrm{CDCl}_{3}, 400 \mathrm{MHz}\right) \delta 7.37-7.25(\mathrm{~m}, 10 \mathrm{H}), 6.07(\mathrm{~s}, 1 \mathrm{H}), 4.20(\mathrm{~d}, J=13.7,1 \mathrm{H}), 3.77(\mathrm{~d}, J=13.7,1 \mathrm{H})$, $3.48(\mathrm{~d}, J=9.7,1 \mathrm{H}), 2.32(\mathrm{dsept}, J=9.7,6.5,1 \mathrm{H}), 1.11(\mathrm{~s}, 9 \mathrm{H}), 1.01983(\mathrm{~s}, 9 \mathrm{H}), 1.022(\mathrm{~d}, J=6.3,3 \mathrm{H}), 0.76(\mathrm{~d}$, $J=6.8,3 \mathrm{H}) ;{ }^{13} \mathrm{C} \mathrm{NMR}\left(\mathrm{CDCl}_{3}, 125 \mathrm{MHz}\right) \delta 160.2,148.9,139.2,129.3,128.9,128.4,127.7,127.5,127.3$, 127.2, 69.6, 52.5, 34.9, 28.8, 28.5, 21.43, 21.37, 21.1, 21.0; IR (thin film) 3311, 3064, 2855, 1586, $1470 \mathrm{~cm}^{-1}$; HRMS (ES) $m / z$ calcd for $\mathrm{C}_{27} \mathrm{H}_{42} \mathrm{NOSi}(\mathrm{M}+\mathrm{H})^{+}$424.3036, found 424.3030. Anal. Calcd for $\mathrm{C}_{27} \mathrm{H}_{41} \mathrm{NOSi}_{\mathrm{C}} \mathrm{C}$, 76.54; H, 9.75; N, 3.31. Found: C, 76.66; H, 9.59; N, 3.09.
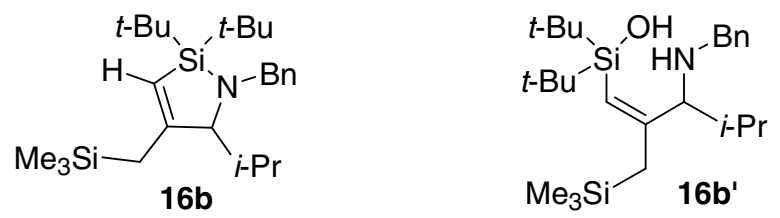

Azasilacyclopentene 16b (NMR Experiment). To an NMR tube were added silaaziridine 6a $(0.50 \mathrm{~mL}, 0.23$ $\mathrm{M}$ in $\left.\mathrm{C}_{7} \mathrm{D}_{8}, 0.12 \mathrm{mmol}\right)$, propargyltrimethylsilane $(21 \mu \mathrm{L}, 0.14 \mathrm{mmol}), \mathrm{PhSiMe}_{3}(0.50 \mathrm{~mL}$ from silaaziridine $6 \mathbf{a}$ stock solution, $0.046 \mathrm{M}, 0.023 \mathrm{mmol})$, and $\mathrm{Pd}\left(\mathrm{PPh}_{3}\right)_{4}(14 \mathrm{mg}, 1 \mathrm{~mol} \%)$. The reaction mixture was heated in an oil bath at $80{ }^{\circ} \mathrm{C}$. After $8 \mathrm{~h}$, azasilacyclopentene $\mathbf{1 6 b}$ was formed in $83 \%$ yield as determined by ${ }^{1} \mathrm{H}$ NMR spectroscopy: ${ }^{1} \mathrm{H}$ NMR $\left(\mathrm{C}_{7} \mathrm{D}_{8}, 400 \mathrm{MHz}\right.$, distinctive peaks) $\delta 5.36(\mathrm{bs}, 1 \mathrm{H}), 4.34(\mathrm{~d}, J=16.1,1 \mathrm{H}), 4.14(\mathrm{~d}, J=$ 16.3, 1H), 3.67 (bs, 1H), 1.15 (s, 9H), 1.13 (s, 9H), 0.98 (d, $J=7.1,3 \mathrm{H}), 0.86(\mathrm{~d}, J=7.4,3 \mathrm{H})$. 
Azasilacyclopentene 16b. To a round-bottom flask were added silaaziridine 6a $(0.36 \mathrm{~g}, 1.2 \mathrm{mmol})$, propargyltrimethylsilane $(0.22 \mathrm{~mL}, 1.5 \mathrm{mmol})$, toluene $(5 \mathrm{~mL}, 0.25 \mathrm{M})$, and $\mathrm{Pd}\left(\mathrm{PPh}_{3}\right)_{4}(14 \mathrm{mg}, 1 \mathrm{~mol} \%)$. The reaction mixture was heated at $80{ }^{\circ} \mathrm{C}$ for $8 \mathrm{~h}$. After $8 \mathrm{~h}$, the reaction mixture was cooled then extracted with hexanes $(10 \mathrm{~mL})$ and $\mathrm{CH}_{3} \mathrm{CN}(2 \times 10 \mathrm{~mL})$ to remove the palladium catalyst. The hexane layers were concentrated in vacuo to give $\mathbf{1 6 b}$ as a pale yellow oil $(0.43 \mathrm{~g}, 85 \%)$. Unpurified $\mathbf{1 6 b}$ was characterized on the basis of ${ }^{1} \mathrm{H}$ and ${ }^{13} \mathrm{C}$ NMR spectral data, IR, and mass spectrometry data: ${ }^{1} \mathrm{H}$ NMR $\left(\mathrm{CDCl}_{3}, 500 \mathrm{MHz}\right) \delta 7.39$ $(\mathrm{m}, 2 \mathrm{H}), 7.32(\mathrm{~m}, 2 \mathrm{H}), 7.21(\mathrm{~m}, 1 \mathrm{H}), 5.34(\mathrm{bs}, 1 \mathrm{H}), 4.38(\mathrm{~d}, J=16.3,1 \mathrm{H}), 4.16(\mathrm{~d}, J=16.3,1 \mathrm{H}), 3.59(\mathrm{bs}, 1 \mathrm{H})$, 2.02 (sept, $J=7.2,1 \mathrm{H}), 1.86(\mathrm{~d}, J=13.6,1 \mathrm{H}), 1.76(\mathrm{~d}, J=13.6,1 \mathrm{H}), 1.063(\mathrm{~s}, 9 \mathrm{H}), 1.056(\mathrm{~s}, 9 \mathrm{H}), 0.86(\mathrm{~d}, J=$ $7.0,3 \mathrm{H}), 0.85(\mathrm{~d}, J=7.4,3 \mathrm{H}), 0.06(\mathrm{~s}, 9 \mathrm{H}) ;{ }^{13} \mathrm{C} \mathrm{NMR}\left(\mathrm{CDCl}_{3}, 125 \mathrm{MHz}\right) \delta 162.5,142.8,128.2,127.8,126.5$, 118.3, 76.2, 52.0, 29.52, 29.48, 29.0, 27.2, 23.2, 22.6, 22.0, 16.8, -0.69; IR (thin film) 3086, 2961, 1567, 1472, $1248 \mathrm{~cm}^{-1}$; HRMS (ES) $\mathrm{m} / \mathrm{z}$ calcd for $\mathrm{C}_{25} \mathrm{H}_{46} \mathrm{NSi}_{2}(\mathrm{M}+\mathrm{H})^{+} 416.3169$, found 416.3176 . Silanol 16b'. Purification of $\mathbf{1 6 b}$ by flash chromatography (84:15:1 hexanes: $\left.\mathrm{CH}_{2} \mathrm{Cl}_{2}: \mathrm{Et}_{3} \mathrm{~N}\right)$ resulted in hydrolysis of $\mathbf{1 6 b}$ to provide 16b' as a white solid (0.28 g, 70\%): $\mathrm{mp} 94{ }^{\circ} \mathrm{C} ;{ }^{1} \mathrm{H} \mathrm{NMR}\left(\mathrm{CDCl}_{3}, 400 \mathrm{MHz}\right) \delta 7.32(\mathrm{~m}, 4 \mathrm{H}), 7.27-7.24$ $(\mathrm{m}, 1 \mathrm{H}), 5.33(\mathrm{~s}, 1 \mathrm{H}), 3.96(\mathrm{~d}, J=12.6,1 \mathrm{H}), 3.67(\mathrm{~d}, J=12.6,1 \mathrm{H}), 2.77(\mathrm{~d}, J=9.2,1 \mathrm{H}), 2.11(\mathrm{dsept}, J=9.1$, $6.7,1 \mathrm{H}), 1.05(\mathrm{~d}, J=6.5,3 \mathrm{H}), 1.01(\mathrm{~s}, 9 \mathrm{H}), 0.98(\mathrm{~d}, J=6.8,3 \mathrm{H}), 0.94(\mathrm{~s}, 9 \mathrm{H}), 0.11(\mathrm{~s}, 9 \mathrm{H}) ;{ }^{13} \mathrm{C} \mathrm{NMR}\left(\mathrm{CDCl}_{3}\right.$, $100 \mathrm{MHz}) \delta 159.5,139.2,128.8,128.0,127.5,119.4,73.2,54.5,36.3,34.8,28.7,28.5,21.7,21.3,21.2,20.8,-$ 0.36; IR (thin film) $3315,3029,2958,1587,1473,1248 \mathrm{~cm}^{-1}$; HRMS (ES) $\mathrm{m} / z$ calcd for $\mathrm{C}_{25} \mathrm{H}_{48} \mathrm{NOSi} \mathrm{N}_{2}(\mathrm{M}+\mathrm{H})^{+}$ 434.3275, found 434.3269. Anal. Calcd for $\mathrm{C}_{25} \mathrm{H}_{47} \mathrm{NOSi}_{2}$ : C, 69.22; H, 10.92; N, 3.23. Found: C, 69.19; H, $10.71 ; \mathrm{N}, 3.21$.
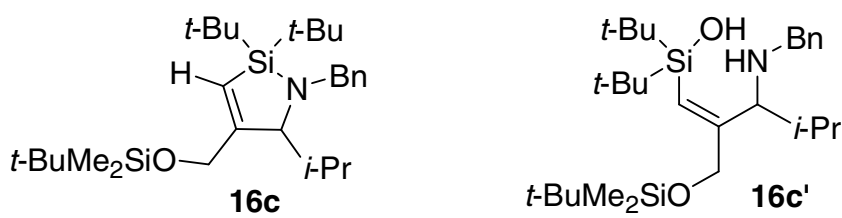

Azasilacyclopentene 16c (NMR Experiment). To an NMR tube were added silaaziridine 6a $(0.50 \mathrm{~mL}, 0.23$ $\mathrm{M}$ in $\left.\mathrm{C}_{6} \mathrm{D}_{6}, 0.12 \mathrm{mmol}\right)$, silyl protected propargyl alcohol $(24 \mathrm{mg}, 0.14 \mathrm{mmol}), \mathrm{PhSiMe}_{3}(0.50 \mathrm{~mL}$ from silaaziridine 6a stock solution, $0.046 \mathrm{M}, 0.023 \mathrm{mmol})$, and $\mathrm{Pd}\left(\mathrm{PPh}_{3}\right)_{4}(14 \mathrm{mg}, 1 \mathrm{~mol} \%)$. The reaction mixture was heated in an oil bath at $80{ }^{\circ} \mathrm{C}$. After $4 \mathrm{~h}$, azasilacyclopentene 16c was formed in $95 \%$ yield as determined by ${ }^{1} \mathrm{H}$ NMR spectroscopy: ${ }^{1} \mathrm{H}$ NMR $\left(\mathrm{C}_{6} \mathrm{D}_{6}, 500 \mathrm{MHz}\right.$, distinctive peaks) $\delta 6.24$ (bd, $\left.J=1.5,1 \mathrm{H}\right), 4.40(\mathrm{~d}, J=$ $16.3,1 \mathrm{H}), 4.31(\mathrm{dd}, J=14.6,1.6,1 \mathrm{H}), 4.16(\mathrm{dd}, J=14.7,1.3,1 \mathrm{H}), 4.11(\mathrm{~d}, J=16.6,1 \mathrm{H}), 3.78(\mathrm{bs}, 1 \mathrm{H}), 2.05$ (septd, $J=7.2,1.8,1 \mathrm{H}), 1.17(\mathrm{~s}, 9 \mathrm{H}), 1.14(\mathrm{~s}, 9 \mathrm{H}), 1.00(\mathrm{~s}, 9 \mathrm{H}), 0.90(\mathrm{~d}, J=6.9,3 \mathrm{H}), 0.81(\mathrm{~d}, J=7.4,3 \mathrm{H}), 0.09$ $(\mathrm{s}, 3 \mathrm{H}), 0.08(\mathrm{~s}, 3 \mathrm{H})$.

Azasilacyclopentene 16c. To a round-bottom flask were added silaaziridine 6a $(0.36 \mathrm{~g}, 1.23 \mathrm{mmol})$, silyl protected propargyl alcohol $(0.26 \mathrm{~mL}, 1.5 \mathrm{mmol})$, toluene $(5 \mathrm{~mL}, 0.25 \mathrm{M})$, and $\mathrm{Pd}\left(\mathrm{PPh}_{3}\right)_{4}(14 \mathrm{mg}, 1 \mathrm{~mol} \%)$. The reaction mixture was heated at $80{ }^{\circ} \mathrm{C}$ for $8 \mathrm{~h}$. After $8 \mathrm{~h}$, the reaction mixture was cooled, then extracted with hexanes $(10 \mathrm{~mL})$ and $\mathrm{CH}_{3} \mathrm{CN}(2 \times 10 \mathrm{~mL})$ to remove the palladium catalyst. The hexane layers were concentrated in vacuo to give $16 \mathrm{c}$ as a pale yellow oil $(0.55 \mathrm{~g}, 94 \%)$. Unpurified 16c was characterized on the basis of ${ }^{1} \mathrm{H}$ and ${ }^{13} \mathrm{C}$ NMR spectral data, IR, and mass spectrometry data: ${ }^{1} \mathrm{H}$ NMR $\left(\mathrm{CDCl}_{3}, 500 \mathrm{MHz}\right) \delta 7.39$ $(\mathrm{m}, 2 \mathrm{H}), 7.31(\mathrm{~m}, 2 \mathrm{H}), 7.21(\mathrm{~m}, 1 \mathrm{H}), 6.01(\mathrm{bs}, 1 \mathrm{H}), 4.40(\mathrm{~d}, J=16.4,1 \mathrm{H}), 4.26(\mathrm{~d}, J=15.0,1 \mathrm{H}), 4.11(\mathrm{~d}, J=$ 15.6, 2H), 3.64 (bs, 1H), 1.93 (sept, $J=7.3,1 \mathrm{H}), 1.07$ (s, 9H), 1.03 (s, 9H), $0.94(\mathrm{~s}, 9 \mathrm{H}), 0.81(\mathrm{~d}, J=7.5,3 \mathrm{H})$, $0.78(\mathrm{~d}, J=7.0,3 \mathrm{H}), 0.09(\mathrm{~s}, 3 \mathrm{H}), 0.08(\mathrm{~s}, 3 \mathrm{H}) ;{ }^{13} \mathrm{C} \mathrm{NMR}\left(\mathrm{CDCl}_{3}, 125 \mathrm{MHz}\right) \delta 163.6,143.0,128.2,127.6$, 126.5, 119.2, 75.0, 65.0, 52.2, 29.4, 29.1, 29.0, 26.2, 23.6, 21.9, 21.1, 18.7, 16.1, -5.0, -5.1; IR (thin film) 3086, 2956, 1472, 1257, $1113 \mathrm{~cm}^{-1}$; HRMS (ES) $\mathrm{m} / z$ calcd for $\mathrm{C}_{28} \mathrm{H}_{52} \mathrm{NOSi}_{2}(\mathrm{M}+\mathrm{H})^{+}$474.3587, found 474.3583. Silanol 16c'. Purification of 16c by flash chromatography (79:20:1 hexanes: $\left.\mathrm{CH}_{2} \mathrm{Cl}_{2}: \mathrm{Et}_{3} \mathrm{~N}\right)$ resulted in hydrolysis of 16c to provide 16c' as a white solid $(0.42 \mathrm{~g}, 70 \%)$ : $\mathrm{mp} 116{ }^{\circ} \mathrm{C} ;{ }^{1} \mathrm{H} \mathrm{NMR}\left(\mathrm{CDCl}_{3}, 500 \mathrm{MHz}\right) \delta$ $8.18(\mathrm{~b}, 1 \mathrm{H}), 7.35-7.25(\mathrm{~m}, 5 \mathrm{H}), 6.06(\mathrm{bdd}, J=1.8,1.8,1 \mathrm{H}), 4.002(\mathrm{dd}, J=15.6,1.8,1 \mathrm{H}), 4.004(\mathrm{~d}, J=13.8$, $1 \mathrm{H}), 3.97(\mathrm{dd}, J=15.5,1.8,1 \mathrm{H}), 3.48(\mathrm{~d}, J=13.8,1 \mathrm{H}), 2.48(\mathrm{~d}, J=10.0,1 \mathrm{H}), 2.25(\mathrm{dsept}, J=9.9,6.6,1 \mathrm{H})$, $2.14(\mathrm{~b}, 1 \mathrm{H}), 1.04(\mathrm{~s}, 9 \mathrm{H}), 1.01(\mathrm{~s}, 9 \mathrm{H}), 0.97(\mathrm{~d}, J=6.4,3 \mathrm{H}), 0.96(\mathrm{~s}, 9 \mathrm{H}), 0.88(\mathrm{~d}, J=6.8,3 \mathrm{H}), 0.11(\mathrm{~s}, 3 \mathrm{H})$, 0.09 (s, 3H); ${ }^{13} \mathrm{C} \mathrm{NMR}\left(\mathrm{CDCl}_{3}, 125 \mathrm{MHz}\right) \delta 158.1,139.3,128.8,127.7,127.4,118.9,69.7,66.9,52.2,33.5$, 
28.7, 28.4, 26.1, 21.4, 21.1, 20.7, 20.5, 18.5, -5.00, -5.03; IR (thin film) 3312, 3027, 2957, 1618, 1472, 1135 $\mathrm{cm}^{-1}$; HRMS (ES) $m / z$ calcd for $\mathrm{C}_{28} \mathrm{H}_{54} \mathrm{NO}_{2} \mathrm{Si}_{2}(\mathrm{M}+\mathrm{H})^{+} 492.3693$, found 492.3703 .

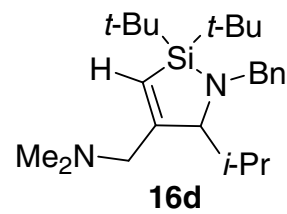

Azasilacyclopentene 16d (NMR Experiment). To an NMR tube were added silaaziridine 6a $(0.50 \mathrm{~mL}, 0.23$ $\mathrm{M}$ in $\left.\mathrm{C}_{7} \mathrm{D}_{8}, 0.12 \mathrm{mmol}\right), 1$-dimethylamino-2-propyne $(15 \mu \mathrm{L}, 0.14 \mathrm{mmol}), \mathrm{PhSiMe}_{3}(0.50 \mathrm{~mL}$ from silaaziridine 6a stock solution, $0.046 \mathrm{M}, 0.023 \mathrm{mmol})$, and $\mathrm{Pd}\left(\mathrm{PPh}_{3}\right)_{4}(14 \mathrm{mg}, 1 \mathrm{~mol} \%)$. The reaction mixture was heated in an oil bath at $80{ }^{\circ} \mathrm{C}$. After $4 \mathrm{~h}$, azasilacyclopentene 16d was formed in $89 \%$ yield as determined by ${ }^{1} \mathrm{H}$ NMR spectroscopy: ${ }^{1} \mathrm{H}$ NMR $\left(\mathrm{C}_{7} \mathrm{D}_{8}, 500 \mathrm{MHz}\right.$, distinctive peaks $) \delta 5.96(\mathrm{bd}, J=1.3,1 \mathrm{H}), 4.36(\mathrm{~d}, J=16.3,1 \mathrm{H}), 4.13$ $(\mathrm{d}, J=16.3,1 \mathrm{H}), 3.87(\mathrm{bs}, 1 \mathrm{H}), 3.00(\mathrm{~d}, J=13.1,1 \mathrm{H}), 2.78(\mathrm{~d}, J=13.9,1 \mathrm{H}), 2.11(\mathrm{~s}, 6 \mathrm{H}), 1.14(\mathrm{~s}, 9 \mathrm{H}), 1.12(\mathrm{~s}$, 9H), $0.94(\mathrm{~d}, J=7.4,3 \mathrm{H}), 0.93(\mathrm{~d}, J=7.1,3 \mathrm{H})$.

Azasilacyclopentene 16d. To a round-bottom flask were added silaaziridine $6 \mathbf{a}(0.36 \mathrm{~g}, 1.2 \mathrm{mmol})$, 1dimethylamino-2-propyne $(0.16 \mathrm{~mL}, 1.5 \mathrm{mmol})$, toluene $(5 \mathrm{~mL}, 0.25 \mathrm{M})$, and $\mathrm{Pd}\left(\mathrm{PPh}_{3}\right)_{4}(14 \mathrm{mg}, 1 \mathrm{~mol} \%)$. The reaction mixture was heated at $80{ }^{\circ} \mathrm{C}$ for $8 \mathrm{~h}$. After $8 \mathrm{~h}$, the reaction mixture was cooled, then extracted with hexanes $(10 \mathrm{~mL})$ and $\mathrm{CH}_{3} \mathrm{CN}(2 \mathrm{x} 10 \mathrm{~mL})$ to remove the palladium catalyst. The hexane layers were concentrated in vacuo to give $\mathbf{1 6 d}$ as a pale yellow oil $(0.41 \mathrm{~g}, 87 \%)$. Purification by flash chromatography (99:1 hexanes: $\left.\mathrm{Et}_{3} \mathrm{~N}\right)$ resulted in partial hydrolysis, but $\mathbf{1 6 d}$ was isolated as a clear oil $(0.34 \mathrm{~g}, 72 \%)$ : ${ }^{1} \mathrm{H} \mathrm{NMR}$ $\left(\mathrm{CDCl}_{3}, 400 \mathrm{MHz}\right) \delta 7.37(\mathrm{~m}, 2 \mathrm{H}), 7.29(\mathrm{~m}, 2 \mathrm{H}), 7.19(\mathrm{~m}, 1 \mathrm{H}), 5.88(\mathrm{bd}, J=1.4,1 \mathrm{H}), 4.37(\mathrm{~d}, J=16.4,1 \mathrm{H})$, $4.13(\mathrm{~d}, J=16.4,1 \mathrm{H}), 3.70(\mathrm{bs}, 1 \mathrm{H}), 2.96(\mathrm{~d}, J=14.4,1 \mathrm{H}), 2.91(\mathrm{~d}, J=14.7,1 \mathrm{H}), 2.23(\mathrm{~s}, 6 \mathrm{H}), 1.99(\mathrm{septd}, J=$ $7.2,1.7,1 \mathrm{H}), 1.05(\mathrm{~s}, 9 \mathrm{H}), 1.04(\mathrm{~s}, 9 \mathrm{H}), 0.86(\mathrm{~d}, J=7.4,3 \mathrm{H}), 0.80(\mathrm{~d}, J=7.0,3 \mathrm{H}) ;{ }^{13} \mathrm{C} \mathrm{NMR}\left(\mathrm{CDCl}_{3}, 100\right.$ $\mathrm{MHz}) \delta 161.7,142.9,128.2,127.7,126.4,123.2,75.0,64.8,52.1,46.2,29.5,29.3,29.1,23.5,22.0,21.1,16.7$; IR (thin film) 3086, 2965, 1584, 1472, $\mathrm{cm}^{-1}$; HRMS (ES) $\mathrm{m} / z$ calcd for $\mathrm{C}_{24} \mathrm{H}_{43} \mathrm{~N}_{2} \mathrm{Si}(\mathrm{M}+\mathrm{H})^{+} 387.3195$, found 387.3183. Anal. Calcd for $\mathrm{C}_{24} \mathrm{H}_{42} \mathrm{~N}_{2} \mathrm{Si}$ : C, 74.54; H, 10.95; N, 7.24. Found: C, 74.49; H, 11.07; N, 7.30.

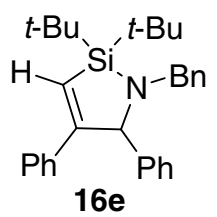

Azasilacyclopentene 16e (NMR Experiment). To an NMR tube were added silaaziridine 6c $(0.50 \mathrm{~mL}, 0.23 \mathrm{M}$ in $\left.\mathrm{C}_{7} \mathrm{D}_{8}, 0.12 \mathrm{mmol}\right)$, phenylacetylene $(15 \mu \mathrm{L}, 0.14 \mathrm{mmol}), \mathrm{PhSiMe}_{3}(0.50 \mathrm{~mL}$ from silaaziridine $6 \mathbf{c}$ stock solution, $0.046 \mathrm{M}, 0.023 \mathrm{mmol})$, and $\mathrm{Pd}\left(\mathrm{PPh}_{3}\right)_{4}(14 \mathrm{mg}, 1 \mathrm{~mol} \%)$. The reaction mixture was heated in an oil bath at $50{ }^{\circ} \mathrm{C}$. After $8 \mathrm{~h}$, azasilacyclopentene 16e was formed in $97 \%$ yield as determined by ${ }^{1} \mathrm{H}$ NMR spectroscopy: ${ }^{1} \mathrm{H}$ NMR $\left(\mathrm{C}_{7} \mathrm{D}_{8}, 400 \mathrm{MHz}\right.$, distinctive peaks) $\delta 6.40(\mathrm{~d}, J=1.6,1 \mathrm{H}), 5.50(\mathrm{~d}, J=1.5,1 \mathrm{H}), 4.24$ $(\mathrm{d}, J=16.1,1 \mathrm{H}), 3.90(\mathrm{~d}, J=16.1,1 \mathrm{H}), 1.19(\mathrm{~s}, 9 \mathrm{H}), 1.18(\mathrm{~s}, 9 \mathrm{H})$.

Azasilacyclopentene 16e. To a round-bottom flask were added silaaziridine $6 \mathbf{c}(0.36 \mathrm{~g}, 1.2 \mathrm{mmol})$, phenylacetylene $(0.16 \mathrm{~mL}, 1.5 \mathrm{mmol})$, toluene $(5 \mathrm{~mL}, 0.25 \mathrm{M})$, and $\mathrm{Pd}\left(\mathrm{PPh}_{3}\right)_{4}(14 \mathrm{mg}, 1 \mathrm{~mol} \%)$. The reaction was stirred at $50{ }^{\circ} \mathrm{C}$ for $8 \mathrm{~h}$. After $8 \mathrm{~h}$, the reaction mixture was cooled, then extracted with hexanes $(10 \mathrm{~mL})$ and $\mathrm{CH}_{3} \mathrm{CN}(2 \times 10 \mathrm{~mL})$ to remove the palladium catalyst. The hexane layers were concentrated in vacuo to give 16e as an orange oil $(0.51 \mathrm{~g}, 95 \%)$. Purification by flash chromatography $(99: 1$ hexanes:Et $3 \mathrm{~N})$ gave 16e as a light orange oil $(0.38 \mathrm{~g}, 70 \%):{ }^{1} \mathrm{H} \mathrm{NMR}\left(\mathrm{CDCl}_{3}, 400 \mathrm{MHz}\right) \delta 7.27-7.23(\mathrm{~m}, 4 \mathrm{H}), 7.20-7.16(\mathrm{~m}, 3 \mathrm{H}), 7.13-$ $7.01(\mathrm{~m}, 6 \mathrm{H}), 6.92-6.90(\mathrm{~m}, 2 \mathrm{H}), 6.47(\mathrm{~d}, J=1.5,1 \mathrm{H}), 5.39(\mathrm{~d}, J=1.3,1 \mathrm{H}), 4.25(\mathrm{~d}, J=16.2,1 \mathrm{H}), 3.81(\mathrm{~d}, J=$ $16.2,1 \mathrm{H}), 1.14(\mathrm{~s}, 9 \mathrm{H}), 1.12(\mathrm{~s}, 9 \mathrm{H}) ;{ }^{13} \mathrm{C} \mathrm{NMR}\left(\mathrm{CDCl}_{3}, 100 \mathrm{MHz}\right) \delta 161.6,141.5,140.5,138.8,129.9,128.2$, 128.1, 127.9, 127.8, 127.5, 127.1, 126.7, 126.4, 123.7, 71.3, 50.3, 29.8, 29.0, 23.1, 22.1; IR (thin film) 3027, 2854, 1557, $1493 \mathrm{~cm}^{-1}$; HRMS (ES) $m / z$ calcd for $\mathrm{C}_{30} \mathrm{H}_{38} \mathrm{NSi}(\mathrm{M}+\mathrm{H})^{+} 440.2773$, found 440.2754. 


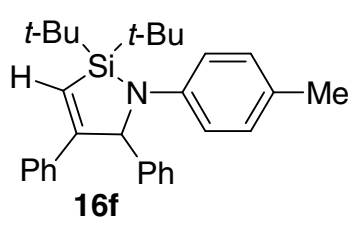

Azasilacyclopentene 16f (NMR Experiment). To an NMR tube were added silaaziridine 6d $(0.50 \mathrm{~mL}, 0.23 \mathrm{M}$ in $\left.\mathrm{C}_{6} \mathrm{D}_{6}, 0.12 \mathrm{mmol}\right)$, phenylacetylene $(15 \mu \mathrm{L}, 0.14 \mathrm{mmol}), \mathrm{PhSiMe}_{3}(0.50 \mathrm{~mL}$ from silaaziridine 6c stock solution, $0.046 \mathrm{M}, 0.023 \mathrm{mmol})$, and $\mathrm{Pd}\left(\mathrm{PPh}_{3}\right)_{4}(14 \mathrm{mg}, 1 \mathrm{~mol} \%)$. The reaction mixture was heated in an oil bath at $50{ }^{\circ} \mathrm{C}$. After $8 \mathrm{~h}$, azasilacyclopentene $\mathbf{1 6 f}$ was formed in $95 \%$ yield as determined by ${ }^{1} \mathrm{H}$ NMR spectroscopy: ${ }^{1} \mathrm{H}$ NMR $\left(\mathrm{C}_{6} \mathrm{D}_{6}, 500 \mathrm{MHz}\right.$, distinctive peaks $) \delta 6.17(\mathrm{~d}, J=1.8,1 \mathrm{H}), 5.90(\mathrm{~d}, J=1.7,1 \mathrm{H}), 2.05$ (s, 3H), 1.40 (s, 9H), 1.07 (s, 9H).

Azasilacyclopentene 16f. To a round-bottom flask were added silaaziridine $6 \mathrm{~d}(0.36 \mathrm{~g}, 1.2 \mathrm{mmol})$, phenylacetylene $(0.16 \mathrm{~mL}, 1.5 \mathrm{mmol})$, toluene $(5 \mathrm{~mL}, 0.25 \mathrm{M})$, and $\mathrm{Pd}\left(\mathrm{PPh}_{3}\right)_{4}(14 \mathrm{mg}, 1 \mathrm{~mol} \%)$. The reaction was stirred at $50{ }^{\circ} \mathrm{C}$ for $8 \mathrm{~h}$. After $8 \mathrm{~h}$, the reaction mixture was cooled, then extracted with hexanes $(10 \mathrm{~mL})$ and $\mathrm{CH}_{3} \mathrm{CN}(2 \times 10 \mathrm{~mL})$ to remove the palladium catalyst. The hexane layers were concentrated in vacuo to give $16 \mathbf{f}$ as a crude yellow oil $(0.53 \mathrm{~g}, 98 \%)$. Purification by flash chromatography (99:1 hexanes:Et $3 \mathrm{~N}) \mathrm{gave}$ 16f as a bright yellow oil $(0.33 \mathrm{~g}, 62 \%)$ : ${ }^{1} \mathrm{H} \mathrm{NMR}\left(\mathrm{CDCl}_{3}, 400 \mathrm{MHz}\right) \delta 7.28-6.82(\mathrm{~m}, 14 \mathrm{H}), 6.18(\mathrm{~d}, J=1.9$, $1 \mathrm{H}), 5.83(\mathrm{~d}, J=1.8,1 \mathrm{H}), 2.17(\mathrm{~s}, 3 \mathrm{H}), 1.37(\mathrm{~s}, 9 \mathrm{H}), 0.96(\mathrm{~s}, 9 \mathrm{H}) ;{ }^{13} \mathrm{C} \mathrm{NMR}\left(\mathrm{CDCl}_{3}, 125 \mathrm{MHz}\right) \delta 162.6,144.7$, 141.5, 139.8, 129.4, 128.7, 128.2, 128.1, 127.9, 127.6, 127.3, 126.9, 123.3, 119.7, 71.5, 30.3, 28.9, 24.1, 22.7, 20.6; IR (thin film) 3061, 3026, 2931, 2856, 1615, 1513, 1472, 1388, 1364, 1271, 1216, 1066, 938, 818, 755, $\mathrm{cm}^{-1}$; HRMS (ES) $\mathrm{m} / \mathrm{z}$ calcd for $\mathrm{C}_{30} \mathrm{H}_{37} \mathrm{NSiNa}(\mathrm{M}+\mathrm{Na})^{+}$462.2593, found 462.2584. Anal. Calcd for $\mathrm{C}_{30} \mathrm{H}_{37} \mathrm{NSi}$ : C, 81.95; H, 8.48; N, 3.19. Found: C, 81.66; H, 8.64; N, 3.39.

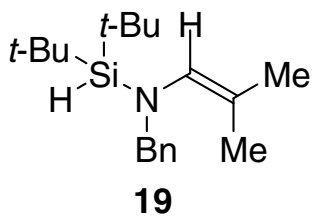

Silane 19. To a J. Young NMR tube were added silaaziridine 6a $\left(0.25 \mathrm{~mL}, 0.46 \mathrm{M}\right.$ in $\left.\mathrm{C}_{6} \mathrm{D}_{6}, 0.12 \mathrm{mmol}\right)$, $\mathrm{Pd}\left(\mathrm{PPh}_{3}\right)_{4}(7 \mathrm{mg}, 5 \mathrm{~mol} \%)$ and $\mathrm{PhSiMe}_{3}(0.25 \mathrm{~mL}$ from silaaziridine 6a stock solution, $0.092 \mathrm{M}, 0.023 \mathrm{mmol})$. The reaction mixture was heated in an oil bath at $100{ }^{\circ} \mathrm{C}$ oil bath and monitored by ${ }^{1} \mathrm{H}$ NMR spectroscopy. After 5 days, silane 19 was formed in $34 \%$ yield as determined by ${ }^{1} \mathrm{H}$ NMR spectroscopy: ${ }^{1} \mathrm{H}$ NMR $\left(\mathrm{C}_{6} \mathrm{D}_{6}, 400\right.$ $\mathrm{MHz}$, distinctive peaks) $\delta 5.55(\mathrm{bs}, 1 \mathrm{H}), 4.20(\mathrm{~s}, 2 \mathrm{H}), 4.13(\mathrm{~s}, 1 \mathrm{H}), 1.80(\mathrm{~s}, 3 \mathrm{H}), 1.52(\mathrm{~s}, 3 \mathrm{H}), 1.12(\mathrm{~s}, 18 \mathrm{H})$.

\section{Protodesilylation of Azasilacyclopentene 16a}

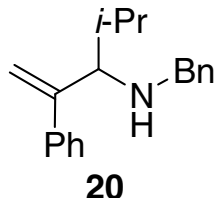

Allylic amine 20. To a mixture of $\mathrm{KO} t$ - $\mathrm{Bu}(0.31 \mathrm{~g}, 3.0 \mathrm{mmol})$ and toluene $(14 \mathrm{~mL})$ was added compound $16 \mathbf{a}$ $(0.41 \mathrm{~g}, 1.0 \mathrm{mmol})$, followed by $6.0 \mathrm{~mL}$ of a $1.0 \mathrm{M}$ solution of $n-\mathrm{Bu}_{4} \mathrm{NF}$ in THF. The reaction mixture was heated at $80{ }^{\circ} \mathrm{C}$ in an oil bath. After $31 \mathrm{~h}$, the reaction mixture was cooled, diluted with $\mathrm{H}_{2} \mathrm{O}(10 \mathrm{~mL})$ and extracted with MTBE $(3 \times 10 \mathrm{~mL})$. The organic extracts were rinsed with $\mathrm{H}_{2} \mathrm{O}(3 \times 10 \mathrm{~mL})$, dried with $\mathrm{MgSO}_{4}$, and concentrated in vacuo. Purification by flash chromatography (89:10:1 hexanes:EtOAc:Et $\left.{ }_{3} \mathrm{~N}\right)$ provided allylic amine 20 as a clear liquid $(0.19 \mathrm{~g}, 72 \%):{ }^{1} \mathrm{H}$ NMR $\left(\mathrm{CDCl}_{3}, 400 \mathrm{MHz}\right) \delta 7.42-7.23(\mathrm{~m}, 10 \mathrm{H}), 5.38(\mathrm{~d}, J=$ 1.7, 1H), 5.22 (bs, 1H), $3.95(\mathrm{~d}, J=13.2,1 \mathrm{H}), 3.67(\mathrm{~d}, J=13.2,1 \mathrm{H}), 3.29$ (d, $J=6.4,1 \mathrm{H}), 1.68$ (octet, $J=6.7$, $1 \mathrm{H}), 1.39(\mathrm{~s}, 1 \mathrm{H}), 0.89(\mathrm{~s}, 3 \mathrm{H}), 0.87(\mathrm{~s}, 3 \mathrm{H}) ;{ }^{13} \mathrm{C} \mathrm{NMR}\left(\mathrm{CDCl}_{3}, 125 \mathrm{MHz}\right) \delta 149.5,142.4,141.4,128.5,128.43$, 128.37, 127.41, 127.39, 127.0, 115.0, 68.6, 51.8, 31.5, 20.6, 18.5; IR (thin film) 3347, 3080, 2957, 1600, 1454 $\mathrm{cm}^{-1}$; HRMS (ES) $\mathrm{m} / z$ calcd for $\mathrm{C}_{19} \mathrm{H}_{24} \mathrm{~N}(\mathrm{M}+\mathrm{H})^{+}$266.1909, found 266.1903. Anal. Calcd for $\mathrm{C}_{19} \mathrm{H}_{23} \mathrm{~N}$ : $\mathrm{C}_{\text {, }}$ 85.99; H, 8.74; N, 5.28. Found: C, 86.06; H, 8.93; N, 5.24. 


\section{References}

(1) Driver, T. G.; Franz, A. K.; Woerpel, K. A. J. Am. Chem. Soc. 2002, 124, 6524-6525.

(2) A general procedure for the synthesis of imines 1, 5c-e, \& $\mathbf{8}$ was followed from: Ishihara, K.; Miyata, M.; Hattori, K.; Tada, T.; Yamamoto, H. J. Am. Chem. Soc. 1994, 116, 10520-10524. Spectroscopic data for imines 1 \& 5c-e can be found at: Nongkunsarn, P.; Ramsden, C. A. Tetrahedron, 1997, 53, 3805-3830. Spectroscopic data for imine 8 can be found at: Beers, O. C. P.; Elsevier, C. J. Ernsting, J.-M. Organometallics 1992, 11, 3886-3893. For preparation of imine 5a, see: Joly, G. D.; Jacobsen, E. N. J. Am. Chem. Soc. 2004, 126, 4102-4103. For preparation of imine 5b, see: Brookes, K. B.; Hickmott, P. W. S. African J. Chem. 1989, 42, 120-124. For preparation of imine 11, see: Wenzel, A. G.; Jacobsen, E. N. J. Am. Chem. Soc. 2002, 124, 12964-12965.

(3) Bodnar, P. M.; Palmer, W. S.; Ridgway, B. H.; Shaw, J. T.; Smitrovich, J. H.; Woerpel, K. A. J. Org. Chem. 1997, 62, 4737-4745.

\section{X-Ray Crystallographic Data}
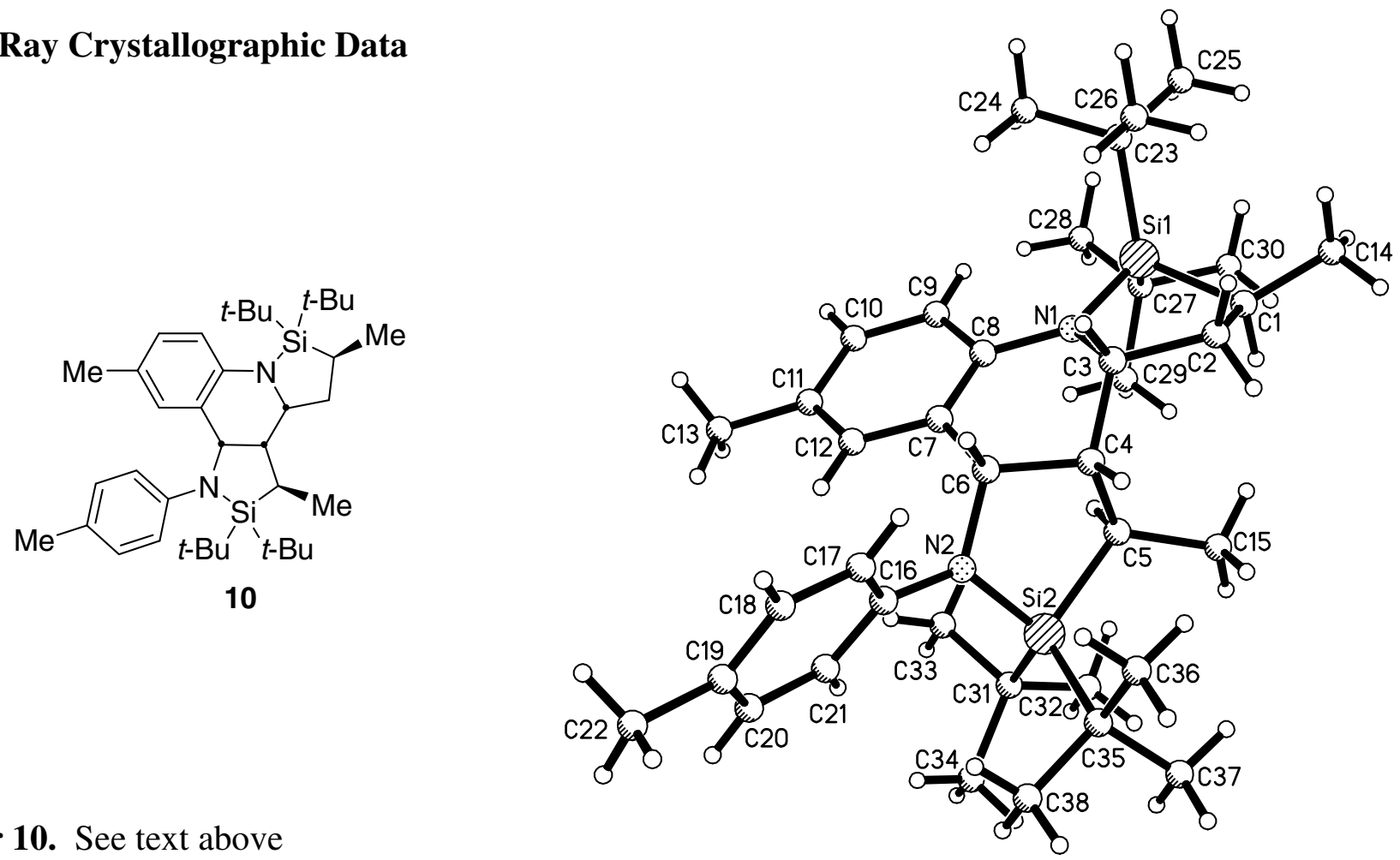

Dimer 10. See text above

X-ray Data Collection, Structure Solution and Refinement for $\mathbf{1 0 .}$

A colorless crystal of approximate dimensions $0.24 \times 0.33 \times 0.35 \mathrm{~mm}$ was mounted on a glass fiber and transferred to a Bruker CCD platform diffractometer. The SMART ${ }^{1}$ program package was used to determine the unit-cell parameters and for data collection (25 sec/frame scan time for a sphere of diffraction data). The raw frame data was processed using $\mathrm{SAINT}^{2}$ and $\mathrm{SADABS}^{3}$ to yield the reflection data file. Subsequent calculations were carried out using the SHELXTL ${ }^{4}$ program. The diffraction symmetry was $2 / m$ and the systematic absences were consistent with the monoclinic space groups $C c$ and $C 2 / c$. It was later determined that the centrosymmetric space group $C 2 / c$ was correct.

The structure was solved by direct methods and refined on $\mathrm{F}^{2}$ by full-matrix least-squares techniques. The analytical scattering factors ${ }^{5}$ for neutral atoms were used throughout the analysis. Hydrogen atoms were located from a difference-Fourier map and refined ( $\mathrm{x}, \mathrm{y}, \mathrm{z}$ and $\left.\mathrm{U}_{\mathrm{iso}}\right)$. 
At convergence, $w R 2=0.1026$ and Goof $=1.050$ for 627 variables refined against 8649 data $(0.76 \AA), \mathrm{R} 1=$ 0.0366 for those 7109 data with $\mathrm{I}>2.0 \sigma(\mathrm{I})$.

\section{References.}

1. SMART Software Users Guide, Version 5.1, Bruker Analytical X-Ray Systems, Inc.; Madison,WI 1999.

2. SAINT Software Users Guide, Version 6.0, Bruker Analytical X-Ray Systems, Inc.; Madison, WI 1999.

3. Sheldrick, G. M. SADABS, Version 2.10, Bruker Analytical X-Ray Systems, Inc.; Madison, WI 2002.

4. Sheldrick, G. M. SHELXTL Version 6.12, Bruker Analytical X-Ray Systems, Inc.; Madison, WI 2001.

5. International Tables for X-Ray Crystallography 1992, Vol. C., Dordrecht: Kluwer Academic Publishers.

Definitions:

$\mathrm{wR} 2=\left[\Sigma\left[\mathrm{w}\left(\mathrm{F}_{\mathrm{o}}^{2}-\mathrm{F}_{\mathrm{c}}^{2}\right)^{2}\right] / \Sigma\left[\mathrm{w}\left(\mathrm{F}_{\mathrm{o}}^{2}\right)^{2}\right]\right]^{1 / 2}$

$\mathrm{R} 1=\Sigma\left\|\mathrm{F}_{\mathrm{o}}|-| \mathrm{F}_{\mathrm{c}}\right\| / \Sigma\left|\mathrm{F}_{\mathrm{o}}\right|$

Goof $=\mathrm{S}=\left[\Sigma\left[\mathrm{w}\left(\mathrm{F}_{\mathrm{o}}^{2}-\mathrm{F}_{\mathrm{c}}^{2}\right)^{2}\right] /(\mathrm{n}-\mathrm{p})\right]^{1 / 2}$ where $\mathrm{n}$ is the number of reflections and $\mathrm{p}$ is the total number of parameters refined.

The thermal ellipsoid plot is shown at the $50 \%$ probability level.

Table 1. Crystal data and structure refinement for $\mathbf{1 0 .}$

Identification code

Empirical formula

Formula weight

Temperature

Wavelength

Crystal system

Space group

Unit cell dimensions

Volume

Z

Density (calculated)

Absorption coefficient

$\mathrm{F}(000)$

Crystal color

Crystal size
10 (Zulimar Nevarez)

$\mathrm{C}_{38} \mathrm{H}_{62} \mathrm{~N}_{2} \mathrm{Si}_{2}$

603.08

163(2) K

$0.71073 \AA$

Monoclinic

$C 2 / c$

$\mathrm{a}=33.179(3) \AA \quad \alpha=90^{\circ}$.

$\mathrm{b}=9.0270(8) \AA$

$\beta=118.7590(10)^{\circ}$.

$\mathrm{c}=27.720(3) \AA$

$\gamma=90^{\circ}$.
8

$1.101 \mathrm{Mg} / \mathrm{m}^{3}$

$0.125 \mathrm{~mm}^{-1}$

2656

colorless

$0.35 \times 0.33 \times 0.24 \mathrm{~mm}^{3}$ 
Theta range for data collection

Index ranges

Reflections collected

Independent reflections

Completeness to theta $=27.88^{\circ}$

Absorption correction

Max. and min. transmission

Refinement method

Data / restraints / parameters

Goodness-of-fit on $\mathrm{F}^{2}$

Final $\mathrm{R}$ indices $[\mathrm{I}>2 \operatorname{sigma}(\mathrm{I})=7109$ data $]$

$\mathrm{R}$ indices (all data; $0.76 \AA$ )

Largest diff. peak and hole
1.58 to $27.88^{\circ}$

$-43 \leq h \leq 43,-11 \leq k \leq 11,-36 \leq l \leq 36$

38468

$8649[\mathrm{R}($ int $)=0.0309]$

$99.6 \%$

Semi-empirical from equivalents

0.9707 and 0.9576

Full-matrix least-squares on $\mathrm{F}^{2}$

8649 / 0 / 627

1.050

$\mathrm{R} 1=0.0366, \mathrm{wR} 2=0.0938$

$\mathrm{R} 1=0.0482, \mathrm{wR} 2=0.1026$

0.432 and -0.236 e. $\AA^{-3}$

Table 2. Atomic coordinates ( x 104) and equivalent isotropic displacement parameters $\left(\AA^{2} \times 10^{3}\right)$ for 10. $U(\mathrm{eq})$ is defined as one third of the trace of the orthogonalized $\mathrm{U}^{\mathrm{ij}}$ tensor.

\begin{tabular}{|c|c|c|c|c|}
\hline & $\mathrm{X}$ & $\mathrm{y}$ & $\mathrm{z}$ & $\mathrm{U}(\mathrm{eq})$ \\
\hline $\operatorname{Si}(1)$ & $1695(1)$ & $12360(1)$ & $6260(1)$ & $17(1)$ \\
\hline $\operatorname{Si}(2)$ & $1259(1)$ & $9460(1)$ & $4075(1)$ & $18(1)$ \\
\hline $\mathrm{N}(1)$ & $1363(1)$ & $11157(1)$ & $5714(1)$ & $18(1)$ \\
\hline $\mathrm{N}(2)$ & $868(1)$ & $8630(1)$ & $4247(1)$ & 17(1) \\
\hline$C(1)$ & $2251(1)$ & $11524(1)$ & $6354(1)$ & $20(1)$ \\
\hline $\mathrm{C}(2)$ & $2117(1)$ & $9910(2)$ & 6161(1) & $22(1)$ \\
\hline $\mathrm{C}(3)$ & $1602(1)$ & $9760(1)$ & $5729(1)$ & $18(1)$ \\
\hline $\mathrm{C}(4)$ & 1501(1) & $9262(1)$ & $5151(1)$ & $18(1)$ \\
\hline $\mathrm{C}(5)$ & $1615(1)$ & $10297(1)$ & $4790(1)$ & $19(1)$ \\
\hline$C(6)$ & $984(1)$ & $8923(1)$ & $4825(1)$ & $16(1)$ \\
\hline $\mathrm{C}(7)$ & $703(1)$ & $10202(1)$ & $4874(1)$ & $17(1)$ \\
\hline $\mathrm{C}(8)$ & $898(1)$ & $11228(1)$ & $5308(1)$ & $18(1)$ \\
\hline $\mathrm{C}(9)$ & $613(1)$ & $12357(2)$ & $5323(1)$ & $23(1)$ \\
\hline$C(10)$ & $160(1)$ & $12497(2)$ & $4920(1)$ & $23(1)$ \\
\hline $\mathrm{C}(11)$ & $-35(1)$ & 11501(1) & $4484(1)$ & $21(1)$ \\
\hline$C(12)$ & $243(1)$ & $10362(1)$ & $4476(1)$ & $19(1)$ \\
\hline$C(13)$ & $-528(1)$ & $11631(2)$ & $4038(1)$ & $27(1)$ \\
\hline$C(14)$ & 2701(1) & $11610(2)$ & $6897(1)$ & $27(1)$ \\
\hline$C(15)$ & $2129(1)$ & $10556(2)$ & 4992(1) & $28(1)$ \\
\hline
\end{tabular}




\begin{tabular}{|c|c|c|c|c|}
\hline$C(16)$ & $520(1)$ & $7589(1)$ & 3939(1) & $17(1)$ \\
\hline $\mathrm{C}(17)$ & $470(1)$ & $6254(2)$ & $4160(1)$ & $24(1)$ \\
\hline $\mathrm{C}(18)$ & $138(1)$ & $5229(2)$ & $3836(1)$ & $28(1)$ \\
\hline $\mathrm{C}(19)$ & $-156(1)$ & $5478(2)$ & $3279(1)$ & $26(1)$ \\
\hline$C(20)$ & $-112(1)$ & $6812(2)$ & 3061(1) & $26(1)$ \\
\hline $\mathrm{C}(21)$ & $214(1)$ & $7856(2)$ & $3384(1)$ & $24(1)$ \\
\hline $\mathrm{C}(22)$ & $-509(1)$ & $4348(2)$ & $2922(1)$ & $41(1)$ \\
\hline $\mathrm{C}(23)$ & 1591(1) & $12039(2)$ & $6873(1)$ & $24(1)$ \\
\hline$C(24)$ & 1091(1) & $12319(2)$ & $6735(1)$ & $31(1)$ \\
\hline$C(25)$ & $1905(1)$ & $13036(2)$ & $7362(1)$ & $36(1)$ \\
\hline$C(26)$ & 1701(1) & $10411(2)$ & $7058(1)$ & $30(1)$ \\
\hline $\mathrm{C}(27)$ & $1672(1)$ & 14394(1) & $6043(1)$ & $24(1)$ \\
\hline $\mathrm{C}(28)$ & $1320(1)$ & $15411(2)$ & $6091(1)$ & $36(1)$ \\
\hline $\mathrm{C}(29)$ & $1584(1)$ & $14437(2)$ & $5446(1)$ & $33(1)$ \\
\hline $\mathrm{C}(30)$ & $2150(1)$ & $15079(2)$ & $6413(1)$ & $31(1)$ \\
\hline $\mathrm{C}(31)$ & $1030(1)$ & $11049(2)$ & $3547(1)$ & $26(1)$ \\
\hline $\mathrm{C}(32)$ & $1422(1)$ & $12165(2)$ & $3685(1)$ & $37(1)$ \\
\hline C(33) & $649(1)$ & $11875(2)$ & $3606(1)$ & $34(1)$ \\
\hline$C(34)$ & $840(1)$ & $10592(2)$ & $2942(1)$ & $33(1)$ \\
\hline$C(35)$ & $1563(1)$ & $7921(2)$ & $3892(1)$ & $22(1)$ \\
\hline $\mathrm{C}(36)$ & $1768(1)$ & $6798(2)$ & $4366(1)$ & $28(1)$ \\
\hline $\mathrm{C}(37)$ & $1954(1)$ & $8538(2)$ & $3802(1)$ & $31(1)$ \\
\hline $\mathrm{C}(38)$ & $1235(1)$ & $7043(2)$ & $3373(1)$ & $27(1)$ \\
\hline
\end{tabular}

Table 3. Bond lengths $[\AA]$ and angles $\left[{ }^{\circ}\right]$ for $\mathbf{1 0}$.

\begin{tabular}{ll}
\hline $\mathrm{Si}(1)-\mathrm{N}(1)$ & $1.7557(11)$ \\
$\mathrm{Si}(1)-\mathrm{C}(1)$ & $1.8901(13)$ \\
$\mathrm{Si}(1)-\mathrm{C}(23)$ & $1.9133(13)$ \\
$\mathrm{Si}(1)-\mathrm{C}(27)$ & $1.9208(13)$ \\
$\mathrm{Si}(2)-\mathrm{N}(2)$ & $1.7510(10)$ \\
$\mathrm{Si}(2)-\mathrm{C}(5)$ & $1.9070(13)$ \\
$\mathrm{Si}(2)-\mathrm{C}(35)$ & $1.9231(13)$ \\
$\mathrm{Si}(2)-\mathrm{C}(31)$ & $1.9245(14)$ \\
$\mathrm{N}(1)-\mathrm{C}(8)$ & $1.4070(15)$ \\
$\mathrm{N}(1)-\mathrm{C}(3)$ & $1.4791(15)$
\end{tabular}




\begin{tabular}{|c|c|}
\hline $\mathrm{N}(2)-\mathrm{C}(16)$ & $1.4143(15)$ \\
\hline $\mathrm{N}(2)-\mathrm{C}(6)$ & $1.4798(14)$ \\
\hline $\mathrm{C}(1)-\mathrm{C}(14)$ & $1.5313(18)$ \\
\hline $\mathrm{C}(1)-\mathrm{C}(2)$ & $1.5420(18)$ \\
\hline$C(2)-C(3)$ & $1.5511(17)$ \\
\hline $\mathrm{C}(3)-\mathrm{C}(4)$ & $1.5360(16)$ \\
\hline$C(4)-C(6)$ & $1.5361(16)$ \\
\hline$C(4)-C(5)$ & $1.5446(17)$ \\
\hline$C(5)-C(15)$ & $1.5358(17)$ \\
\hline$C(6)-C(7)$ & $1.5292(16)$ \\
\hline$C(7)-C(12)$ & $1.3956(17)$ \\
\hline$C(7)-C(8)$ & $1.4054(17)$ \\
\hline $\mathrm{C}(8)-\mathrm{C}(9)$ & $1.4040(17)$ \\
\hline C(9)-C(10) & $1.3810(19)$ \\
\hline $\mathrm{C}(10)-\mathrm{C}(11)$ & $1.3908(19)$ \\
\hline $\mathrm{C}(11)-\mathrm{C}(12)$ & $1.3887(17)$ \\
\hline $\mathrm{C}(11)-\mathrm{C}(13)$ & $1.5074(18)$ \\
\hline$C(16)-C(21)$ & $1.3973(17)$ \\
\hline$C(16)-C(17)$ & $1.3976(18)$ \\
\hline $\mathrm{C}(17)-\mathrm{C}(18)$ & $1.3870(19)$ \\
\hline C(18)-C(19) & $1.392(2)$ \\
\hline $\mathrm{C}(19)-\mathrm{C}(20)$ & $1.386(2)$ \\
\hline$C(19)-C(22)$ & $1.508(2)$ \\
\hline $\mathrm{C}(20)-\mathrm{C}(21)$ & $1.3873(19)$ \\
\hline $\mathrm{C}(23)-\mathrm{C}(24)$ & $1.535(2)$ \\
\hline $\mathrm{C}(23)-\mathrm{C}(25)$ & $1.5392(19)$ \\
\hline$C(23)-C(26)$ & $1.541(2)$ \\
\hline $\mathrm{C}(27)-\mathrm{C}(29)$ & $1.537(2)$ \\
\hline $\mathrm{C}(27)-\mathrm{C}(28)$ & $1.5375(19)$ \\
\hline $\mathrm{C}(27)-\mathrm{C}(30)$ & $1.546(2)$ \\
\hline $\mathrm{C}(31)-\mathrm{C}(34)$ & $1.539(2)$ \\
\hline $\mathrm{C}(31)-\mathrm{C}(32)$ & $1.541(2)$ \\
\hline $\mathrm{C}(31)-\mathrm{C}(33)$ & $1.542(2)$ \\
\hline $\mathrm{C}(35)-\mathrm{C}(36)$ & $1.5369(19)$ \\
\hline $\mathrm{C}(35)-\mathrm{C}(37)$ & $1.5386(19)$ \\
\hline$C(35)-C(38)$ & $1.5406(19)$ \\
\hline $\mathrm{N}(1)-\mathrm{Si}(1)-\mathrm{C}(1)$ & $92.18(5)$ \\
\hline $\mathrm{N}(1)-\mathrm{Si}(1)-\mathrm{C}(23)$ & $111.45(6)$ \\
\hline
\end{tabular}




\begin{tabular}{|c|c|}
\hline $\mathrm{C}(1)-\mathrm{Si}(1)-\mathrm{C}(23)$ & $113.44(6)$ \\
\hline $\mathrm{N}(1)-\mathrm{Si}(1)-\mathrm{C}(27)$ & $114.08(6)$ \\
\hline $\mathrm{C}(1)-\mathrm{Si}(1)-\mathrm{C}(27)$ & $108.37(6)$ \\
\hline $\mathrm{C}(23)-\mathrm{Si}(1)-\mathrm{C}(27)$ & $115.13(6)$ \\
\hline $\mathrm{N}(2)-\mathrm{Si}(2)-\mathrm{C}(5)$ & $93.19(5)$ \\
\hline $\mathrm{N}(2)-\mathrm{Si}(2)-\mathrm{C}(35)$ & $108.31(5)$ \\
\hline $\mathrm{C}(5)-\mathrm{Si}(2)-\mathrm{C}(35)$ & $114.78(6)$ \\
\hline $\mathrm{N}(2)-\mathrm{Si}(2)-\mathrm{C}(31)$ & $116.98(5)$ \\
\hline$C(5)-\operatorname{Si}(2)-C(31)$ & $108.38(6)$ \\
\hline $\mathrm{C}(35)-\mathrm{Si}(2)-\mathrm{C}(31)$ & $113.72(6)$ \\
\hline $\mathrm{C}(8)-\mathrm{N}(1)-\mathrm{C}(3)$ & $115.37(10)$ \\
\hline $\mathrm{C}(8)-\mathrm{N}(1)-\mathrm{Si}(1)$ & $130.79(8)$ \\
\hline $\mathrm{C}(3)-\mathrm{N}(1)-\mathrm{Si}(1)$ & $113.40(8)$ \\
\hline $\mathrm{C}(16)-\mathrm{N}(2)-\mathrm{C}(6)$ & $118.98(9)$ \\
\hline $\mathrm{C}(16)-\mathrm{N}(2)-\mathrm{Si}(2)$ & $128.04(8)$ \\
\hline $\mathrm{C}(6)-\mathrm{N}(2)-\mathrm{Si}(2)$ & $112.21(8)$ \\
\hline $\mathrm{C}(14)-\mathrm{C}(1)-\mathrm{C}(2)$ & $111.75(11)$ \\
\hline$C(14)-C(1)-S i(1)$ & $122.74(9)$ \\
\hline $\mathrm{C}(2)-\mathrm{C}(1)-\mathrm{Si}(1)$ & $103.36(8)$ \\
\hline $\mathrm{C}(1)-\mathrm{C}(2)-\mathrm{C}(3)$ & $112.08(10)$ \\
\hline $\mathrm{N}(1)-\mathrm{C}(3)-\mathrm{C}(4)$ & $112.01(9)$ \\
\hline $\mathrm{N}(1)-\mathrm{C}(3)-\mathrm{C}(2)$ & $108.94(10)$ \\
\hline $\mathrm{C}(4)-\mathrm{C}(3)-\mathrm{C}(2)$ & $115.95(10)$ \\
\hline $\mathrm{C}(3)-\mathrm{C}(4)-\mathrm{C}(6)$ & $107.10(9)$ \\
\hline $\mathrm{C}(3)-\mathrm{C}(4)-\mathrm{C}(5)$ & $120.04(10)$ \\
\hline$C(6)-C(4)-C(5)$ & $106.90(9)$ \\
\hline$C(15)-C(5)-C(4)$ & $115.53(11)$ \\
\hline$C(15)-C(5)-S i(2)$ & $117.59(9)$ \\
\hline$C(4)-C(5)-S i(2)$ & $102.26(8)$ \\
\hline $\mathrm{N}(2)-\mathrm{C}(6)-\mathrm{C}(7)$ & $112.22(9)$ \\
\hline $\mathrm{N}(2)-\mathrm{C}(6)-\mathrm{C}(4)$ & 107.93(9) \\
\hline $\mathrm{C}(7)-\mathrm{C}(6)-\mathrm{C}(4)$ & $110.63(10)$ \\
\hline $\mathrm{C}(12)-\mathrm{C}(7)-\mathrm{C}(8)$ & $119.03(11)$ \\
\hline $\mathrm{C}(12)-\mathrm{C}(7)-\mathrm{C}(6)$ & $119.31(11)$ \\
\hline $\mathrm{C}(8)-\mathrm{C}(7)-\mathrm{C}(6)$ & $121.66(10)$ \\
\hline $\mathrm{C}(9)-\mathrm{C}(8)-\mathrm{C}(7)$ & $117.73(11)$ \\
\hline $\mathrm{C}(9)-\mathrm{C}(8)-\mathrm{N}(1)$ & $120.31(11)$ \\
\hline $\mathrm{C}(7)-\mathrm{C}(8)-\mathrm{N}(1)$ & $121.96(10)$ \\
\hline
\end{tabular}




\begin{tabular}{|c|c|}
\hline$C(10)-C(9)-C(8)$ & $121.97(12)$ \\
\hline$C(9)-C(10)-C(11)$ & $120.82(12)$ \\
\hline$C(12)-C(11)-C(10)$ & $117.32(11)$ \\
\hline$C(12)-C(11)-C(13)$ & $120.69(12)$ \\
\hline $\mathrm{C}(10)-\mathrm{C}(11)-\mathrm{C}(13)$ & $121.99(12)$ \\
\hline$C(11)-C(12)-C(7)$ & $123.10(12)$ \\
\hline$C(21)-C(16)-C(17)$ & $117.09(12)$ \\
\hline $\mathrm{C}(21)-\mathrm{C}(16)-\mathrm{N}(2)$ & $120.07(11)$ \\
\hline $\mathrm{C}(17)-\mathrm{C}(16)-\mathrm{N}(2)$ & $122.83(11)$ \\
\hline$C(18)-C(17)-C(16)$ & $121.02(12)$ \\
\hline$C(17)-C(18)-C(19)$ & $121.69(13)$ \\
\hline$C(20)-C(19)-C(18)$ & $117.29(12)$ \\
\hline$C(20)-C(19)-C(22)$ & $120.93(13)$ \\
\hline$C(18)-C(19)-C(22)$ & $121.77(14)$ \\
\hline$C(19)-C(20)-C(21)$ & $121.51(12)$ \\
\hline$C(20)-C(21)-C(16)$ & $121.34(13)$ \\
\hline $\mathrm{C}(24)-\mathrm{C}(23)-\mathrm{C}(25)$ & $108.44(12)$ \\
\hline$C(24)-C(23)-C(26)$ & $107.13(12)$ \\
\hline$C(25)-C(23)-C(26)$ & $108.31(12)$ \\
\hline$C(24)-C(23)-\operatorname{Si}(1)$ & $112.83(9)$ \\
\hline$C(25)-C(23)-S i(1)$ & $111.04(9)$ \\
\hline$C(26)-C(23)-S i(1)$ & $108.93(9)$ \\
\hline $\mathrm{C}(29)-\mathrm{C}(27)-\mathrm{C}(28)$ & $108.52(12)$ \\
\hline $\mathrm{C}(29)-\mathrm{C}(27)-\mathrm{C}(30)$ & $107.80(12)$ \\
\hline $\mathrm{C}(28)-\mathrm{C}(27)-\mathrm{C}(30)$ & $106.46(12)$ \\
\hline$C(29)-C(27)-S i(1)$ & $108.58(9)$ \\
\hline $\mathrm{C}(28)-\mathrm{C}(27)-\mathrm{Si}(1)$ & $117.60(10)$ \\
\hline $\mathrm{C}(30)-\mathrm{C}(27)-\mathrm{Si}(1)$ & $107.51(9)$ \\
\hline $\mathrm{C}(34)-\mathrm{C}(31)-\mathrm{C}(32)$ & $107.45(12)$ \\
\hline $\mathrm{C}(34)-\mathrm{C}(31)-\mathrm{C}(33)$ & $108.58(12)$ \\
\hline $\mathrm{C}(32)-\mathrm{C}(31)-\mathrm{C}(33)$ & $107.13(13)$ \\
\hline $\mathrm{C}(34)-\mathrm{C}(31)-\mathrm{Si}(2)$ & $115.76(10)$ \\
\hline $\mathrm{C}(32)-\mathrm{C}(31)-\mathrm{Si}(2)$ & $108.74(10)$ \\
\hline $\mathrm{C}(33)-\mathrm{C}(31)-\mathrm{Si}(2)$ & $108.84(9)$ \\
\hline $\mathrm{C}(36)-\mathrm{C}(35)-\mathrm{C}(37)$ & $108.43(11)$ \\
\hline $\mathrm{C}(36)-\mathrm{C}(35)-\mathrm{C}(38)$ & $106.39(12)$ \\
\hline $\mathrm{C}(37)-\mathrm{C}(35)-\mathrm{C}(38)$ & $107.90(11)$ \\
\hline$C(36)-C(35)-S i(2)$ & $108.85(8)$ \\
\hline
\end{tabular}


$\mathrm{C}(37)-\mathrm{C}(35)-\mathrm{Si}(2) \quad 111.85(10)$

$\mathrm{C}(38)-\mathrm{C}(35)-\mathrm{Si}(2) \quad 113.18(9)$

Table 4. Anisotropic displacement parameters $\left(\AA^{2} \times 10^{3}\right)$ for 10. The anisotropic displacement factor exponent takes the form: $-2 \pi^{2}\left[h^{2} a^{* 2} U^{11}+\ldots+2 h k a^{*} b^{*} U^{12}\right]$

\begin{tabular}{|c|c|c|c|c|c|c|}
\hline & $\mathrm{U}^{11}$ & $\mathrm{U}^{22}$ & $\mathrm{U}^{33}$ & $\mathrm{U}^{23}$ & $\mathrm{U}^{13}$ & $\mathrm{U}^{12}$ \\
\hline $\operatorname{Si}(1)$ & $19(1)$ & $15(1)$ & $15(1)$ & $0(1)$ & $8(1)$ & $0(1)$ \\
\hline $\operatorname{Si}(2)$ & $18(1)$ & $19(1)$ & $17(1)$ & $2(1)$ & $10(1)$ & $2(1)$ \\
\hline $\mathrm{N}(1)$ & $18(1)$ & $17(1)$ & $16(1)$ & $-1(1)$ & $7(1)$ & $3(1)$ \\
\hline $\mathrm{N}(2)$ & $18(1)$ & $20(1)$ & $14(1)$ & $-1(1)$ & $9(1)$ & $0(1)$ \\
\hline $\mathrm{C}(1)$ & $19(1)$ & $21(1)$ & $17(1)$ & $2(1)$ & $7(1)$ & $1(1)$ \\
\hline$C(2)$ & $21(1)$ & $21(1)$ & $19(1)$ & $1(1)$ & $4(1)$ & $4(1)$ \\
\hline C(3) & $19(1)$ & $16(1)$ & $16(1)$ & $1(1)$ & $7(1)$ & $3(1)$ \\
\hline$C(4)$ & $18(1)$ & $17(1)$ & $17(1)$ & $0(1)$ & $8(1)$ & $3(1)$ \\
\hline$C(5)$ & $18(1)$ & $20(1)$ & $21(1)$ & $-1(1)$ & $11(1)$ & $0(1)$ \\
\hline$C(6)$ & $19(1)$ & $17(1)$ & $14(1)$ & $0(1)$ & $9(1)$ & $1(1)$ \\
\hline$C(7)$ & $18(1)$ & $18(1)$ & $17(1)$ & $2(1)$ & $10(1)$ & $1(1)$ \\
\hline$C(8)$ & $18(1)$ & $18(1)$ & $17(1)$ & $2(1)$ & $9(1)$ & $1(1)$ \\
\hline $\mathrm{C}(9)$ & $24(1)$ & $21(1)$ & $24(1)$ & $-4(1)$ & $12(1)$ & $2(1)$ \\
\hline$C(10)$ & $22(1)$ & $21(1)$ & $29(1)$ & $1(1)$ & $15(1)$ & $6(1)$ \\
\hline $\mathrm{C}(11)$ & $18(1)$ & $23(1)$ & $23(1)$ & $3(1)$ & $11(1)$ & $2(1)$ \\
\hline$C(12)$ & $19(1)$ & $22(1)$ & $19(1)$ & $-1(1)$ & $11(1)$ & $-1(1)$ \\
\hline$C(13)$ & $18(1)$ & $30(1)$ & $30(1)$ & $2(1)$ & $10(1)$ & $3(1)$ \\
\hline$C(14)$ & $22(1)$ & $28(1)$ & $25(1)$ & $-1(1)$ & $5(1)$ & $-1(1)$ \\
\hline$C(15)$ & $21(1)$ & $40(1)$ & $28(1)$ & $-7(1)$ & $14(1)$ & $-6(1)$ \\
\hline$C(16)$ & $17(1)$ & $19(1)$ & $17(1)$ & $-2(1)$ & $9(1)$ & $2(1)$ \\
\hline$C(17)$ & $28(1)$ & $21(1)$ & $18(1)$ & 1(1) & $7(1)$ & $2(1)$ \\
\hline$C(18)$ & $35(1)$ & $19(1)$ & $27(1)$ & $-1(1)$ & $12(1)$ & $-3(1)$ \\
\hline C(19) & $26(1)$ & $26(1)$ & $25(1)$ & $-8(1)$ & 11(1) & $-3(1)$ \\
\hline $\mathrm{C}(20)$ & $24(1)$ & $34(1)$ & $16(1)$ & $-3(1)$ & $7(1)$ & $-2(1)$ \\
\hline $\mathrm{C}(21)$ & $26(1)$ & $27(1)$ & $18(1)$ & $2(1)$ & $10(1)$ & $-2(1)$ \\
\hline$C(22)$ & $42(1)$ & $34(1)$ & $35(1)$ & $-12(1)$ & $9(1)$ & $-12(1)$ \\
\hline$C(23)$ & $29(1)$ & $26(1)$ & $18(1)$ & $-3(1)$ & $13(1)$ & $-6(1)$ \\
\hline$C(24)$ & $35(1)$ & $36(1)$ & $31(1)$ & $-3(1)$ & $23(1)$ & $-3(1)$ \\
\hline
\end{tabular}




$\begin{array}{llllclc}\mathrm{C}(25) & 44(1) & 42(1) & 22(1) & -11(1) & 17(1) & -13(1) \\ \mathrm{C}(26) & 36(1) & 32(1) & 23(1) & 5(1) & 15(1) & -4(1) \\ \mathrm{C}(27) & 27(1) & 17(1) & 30(1) & 2(1) & 15(1) & 0(1) \\ \mathrm{C}(28) & 37(1) & 17(1) & 58(1) & -1(1) & 26(1) & 2(1) \\ \mathrm{C}(29) & 42(1) & 27(1) & 31(1) & 13(1) & 18(1) & 3(1) \\ \mathrm{C}(30) & 32(1) & 22(1) & 41(1) & 0(1) & 18(1) & -6(1) \\ \mathrm{C}(31) & 31(1) & 24(1) & 26(1) & 7(1) & 16(1) & 4(1) \\ \mathrm{C}(32) & 46(1) & 30(1) & 42(1) & 9(1) & 25(1) & -3(1) \\ \mathrm{C}(33) & 40(1) & 28(1) & 36(1) & 12(1) & 21(1) & 14(1) \\ \mathrm{C}(34) & 42(1) & 34(1) & 25(1) & 12(1) & 18(1) & 6(1) \\ \mathrm{C}(35) & 22(1) & 27(1) & 20(1) & 0(1) & 12(1) & 4(1) \\ \mathrm{C}(36) & 30(1) & 30(1) & 25(1) & 1(1) & 13(1) & 12(1) \\ \mathrm{C}(37) & 27(1) & 41(1) & 32(1) & -6(1) & 20(1) & 0(1) \\ \mathrm{C}(38) & 29(1) & 31(1) & 23(1) & -3(1) & 14(1) & 4(1)\end{array}$

Table 5. Hydrogen coordinates ( x 104) and isotropic displacement parameters $\left(\AA^{2} \times 10^{3}\right)$ for $\mathbf{1 0}$.

\begin{tabular}{|c|c|c|c|c|}
\hline & $\mathrm{x}$ & $\mathrm{y}$ & $\mathrm{z}$ & $\mathrm{U}(\mathrm{eq})$ \\
\hline $\mathrm{H}(1 \mathrm{~A})$ & $2305(5)$ & $12020(17)$ & $6081(6)$ & $24(4)$ \\
\hline $\mathrm{H}(2 \mathrm{~A})$ & $2175(6)$ & $9300(19)$ & $6477(7)$ & $34(4)$ \\
\hline $\mathrm{H}(2 \mathrm{~B})$ & $2311(5)$ & $9494(17)$ & $6009(6)$ & $23(4)$ \\
\hline $\mathrm{H}(3 \mathrm{~A})$ & $1460(5)$ & $8977(16)$ & $5859(6)$ & $15(3)$ \\
\hline $\mathrm{H}(4 \mathrm{~A})$ & $1674(5)$ & $8352(17)$ & $5208(6)$ & $19(4)$ \\
\hline $\mathrm{H}(5 \mathrm{~A})$ & $1472(5)$ & $11253(18)$ & $4773(6)$ & $26(4)$ \\
\hline $\mathrm{H}(6 \mathrm{~A})$ & $924(5)$ & $8016(16)$ & 4988(6) & $18(3)$ \\
\hline $\mathrm{H}(9 \mathrm{~A})$ & $728(5)$ & 13048(18) & $5614(7)$ & $27(4)$ \\
\hline $\mathrm{H}(10 \mathrm{~A})$ & $-20(6)$ & 13280(18) & $4945(7)$ & $30(4)$ \\
\hline $\mathrm{H}(12 \mathrm{~A})$ & $111(5)$ & $9657(17)$ & $4181(6)$ & $24(4)$ \\
\hline $\mathrm{H}(13 \mathrm{~A})$ & $-578(7)$ & $11200(20)$ & $3690(9)$ & $57(6)$ \\
\hline $\mathrm{H}(13 \mathrm{~B})$ & $-615(7)$ & $12690(30)$ & 3943(9) & $58(6)$ \\
\hline $\mathrm{H}(13 \mathrm{C})$ & $-727(8)$ & $11140(30)$ & 4131(9) & $65(6)$ \\
\hline $\mathrm{H}(14 \mathrm{~A})$ & 2941(6) & $11110(20)$ & $6861(7)$ & $38(5)$ \\
\hline $\mathrm{H}(14 \mathrm{~B})$ & $2670(6)$ & 11133(19) & $7204(7)$ & $34(4)$ \\
\hline $\mathrm{H}(14 \mathrm{C})$ & $2801(6)$ & $12620(20)$ & $7015(7)$ & $34(4)$ \\
\hline
\end{tabular}




\begin{tabular}{|c|c|c|c|c|}
\hline $\mathrm{H}(15 \mathrm{~A})$ & $2280(6)$ & $11130(20)$ & $5337(7)$ & $36(4)$ \\
\hline $\mathrm{H}(15 \mathrm{~B})$ & $2174(6)$ & 11116(19) & $4733(7)$ & $34(4)$ \\
\hline $\mathrm{H}(15 \mathrm{C})$ & $2284(6)$ & $9610(20)$ & $5042(7)$ & $41(5)$ \\
\hline $\mathrm{H}(17 \mathrm{~A})$ & $669(6)$ & $6026(19)$ & $4542(7)$ & $30(4)$ \\
\hline $\mathrm{H}(18 \mathrm{~A})$ & $126(6)$ & $4310(20)$ & $4000(7)$ & $38(5)$ \\
\hline $\mathrm{H}(20 \mathrm{~A})$ & $-308(6)$ & 7043(18) & $2673(7)$ & $30(4)$ \\
\hline $\mathrm{H}(21 \mathrm{~A})$ & $224(5)$ & 8774(19) & $3220(7)$ & $29(4)$ \\
\hline $\mathrm{H}(22 \mathrm{~A})$ & $-581(9)$ & $4350(30)$ & $2557(12)$ & $89(9)$ \\
\hline $\mathrm{H}(22 \mathrm{~B})$ & $-797(11)$ & $4510(30)$ & $2908(13)$ & $114(11$ \\
\hline $\mathrm{H}(22 \mathrm{C})$ & $-412(8)$ & $3360(30)$ & $3083(10)$ & $76(7)$ \\
\hline $\mathrm{H}(24 \mathrm{~A})$ & $1054(6)$ & $12120(20)$ & 7064(8) & $43(5)$ \\
\hline $\mathrm{H}(24 \mathrm{~B})$ & $877(6)$ & 11655(19) & $6435(7)$ & $33(4)$ \\
\hline $\mathrm{H}(24 \mathrm{C})$ & $1000(6)$ & $13340(20)$ & $6620(7)$ & $30(4)$ \\
\hline $\mathrm{H}(25 \mathrm{~A})$ & 1853(6) & $12760(20)$ & 7693(8) & $43(5)$ \\
\hline $\mathrm{H}(25 \mathrm{~B})$ & $1829(7)$ & $14140(20)$ & $7268(8)$ & $48(5)$ \\
\hline $\mathrm{H}(25 \mathrm{C})$ & $2231(6)$ & $12910(20)$ & $7462(7)$ & $37(5)$ \\
\hline $\mathrm{H}(26 \mathrm{~A})$ & $1642(6)$ & $10210(20)$ & $7366(8)$ & $42(5)$ \\
\hline $\mathrm{H}(26 \mathrm{~B})$ & $2026(6)$ & $10150(20)$ & $7190(7)$ & $37(5)$ \\
\hline $\mathrm{H}(26 \mathrm{C})$ & $1520(6)$ & $9747(19)$ & $6759(7)$ & $31(4)$ \\
\hline $\mathrm{H}(28 \mathrm{~A})$ & $1374(6)$ & $16430(20)$ & $6004(8)$ & $45(5)$ \\
\hline $\mathrm{H}(28 \mathrm{~B})$ & $1367(6)$ & $15450(20)$ & $6478(8)$ & $43(5)$ \\
\hline $\mathrm{H}(28 \mathrm{C})$ & $998(6)$ & $15140(20)$ & $5848(7)$ & $40(5)$ \\
\hline $\mathrm{H}(29 \mathrm{~A})$ & 1601(6) & $15470(20)$ & $5347(8)$ & $46(5)$ \\
\hline $\mathrm{H}(29 \mathrm{~B})$ & $1268(7)$ & $14050(20)$ & $5173(8)$ & $43(5)$ \\
\hline $\mathrm{H}(29 \mathrm{C})$ & $1828(6)$ & 13847(19) & $5424(7)$ & $32(4)$ \\
\hline $\mathrm{H}(30 \mathrm{~A})$ & $2138(6)$ & $16150(20)$ & $6299(8)$ & $44(5)$ \\
\hline $\mathrm{H}(30 \mathrm{~B})$ & $2396(6)$ & 14559(19) & $6377(7)$ & $33(4)$ \\
\hline $\mathrm{H}(30 \mathrm{C})$ & $2234(6)$ & $15100(20)$ & $6805(8)$ & $40(5)$ \\
\hline $\mathrm{H}(32 \mathrm{~A})$ & $1309(7)$ & $13000(20)$ & $3425(8)$ & $53(6)$ \\
\hline $\mathrm{H}(32 \mathrm{~B})$ & $1677(7)$ & $11750(20)$ & $3660(8)$ & $54(6)$ \\
\hline $\mathrm{H}(32 \mathrm{C})$ & $1542(6)$ & $12600(20)$ & $4053(8)$ & $40(5)$ \\
\hline $\mathrm{H}(33 \mathrm{~A})$ & $556(6)$ & $12750(20)$ & $3377(8)$ & $46(5)$ \\
\hline $\mathrm{H}(33 \mathrm{~B})$ & 763(7) & $12220(20)$ & $4002(8)$ & $47(5)$ \\
\hline $\mathrm{H}(33 \mathrm{C})$ & $366(6)$ & $11250(20)$ & $3481(7)$ & $37(5)$ \\
\hline $\mathrm{H}(34 \mathrm{~A})$ & $724(7)$ & $11470(20)$ & 2711(8) & $49(5)$ \\
\hline $\mathrm{H}(34 \mathrm{~B})$ & $583(6)$ & $9836(19)$ & $2813(7)$ & $32(4)$ \\
\hline $\mathrm{H}(34 \mathrm{C})$ & 1081(6) & 10190(20) & $2880(7)$ & $40(5)$ \\
\hline $\mathrm{H}(36 \mathrm{~A})$ & 1912(6) & $5990(20)$ & $4260(7)$ & $39(5)$ \\
\hline
\end{tabular}




$\begin{array}{lrlll}\mathrm{H}(36 \mathrm{~B}) & 1535(6) & 6365(19) & 4446(7) & 32(4) \\ \mathrm{H}(36 \mathrm{C}) & 2015(6) & 7259(19) & 4710(7) & 34(4) \\ \mathrm{H}(37 \mathrm{~A}) & 2116(6) & 7740(20) & 3727(8) & 45(5) \\ \mathrm{H}(37 \mathrm{~B}) & 2185(6) & 9100(20) & 4116(8) & 40(5) \\ \mathrm{H}(37 \mathrm{C}) & 1830(6) & 9220(20) & 3474(7) & 36(4) \\ \mathrm{H}(38 \mathrm{~A}) & 1419(6) & 6260(19) & 3306(7) & 32(4) \\ \mathrm{H}(38 B) & 1099(6) & 7672(18) & 3047(7) & 30(4) \\ \mathrm{H}(38 \mathrm{C}) & 985(6) & 6577(19) & 3410(7) & 37(5)\end{array}$

Table 6. Torsion angles $\left[{ }^{\circ}\right]$ for $\mathbf{1 0}$.

$\begin{array}{lc}\mathrm{C}(1)-\mathrm{Si}(1)-\mathrm{N}(1)-\mathrm{C}(8) & -163.43(11) \\ \mathrm{C}(23)-\mathrm{Si}(1)-\mathrm{N}(1)-\mathrm{C}(8) & 80.30(12) \\ \mathrm{C}(27)-\mathrm{Si}(1)-\mathrm{N}(1)-\mathrm{C}(8) & -52.17(12) \\ \mathrm{C}(1)-\mathrm{Si}(1)-\mathrm{N}(1)-\mathrm{C}(3) & 24.65(9) \\ \mathrm{C}(23)-\mathrm{Si}(1)-\mathrm{N}(1)-\mathrm{C}(3) & -91.62(9) \\ \mathrm{C}(27)-\mathrm{Si}(1)-\mathrm{N}(1)-\mathrm{C}(3) & 135.91(8) \\ \mathrm{C}(5)-\mathrm{Si}(2)-\mathrm{N}(2)-\mathrm{C}(16) & -170.10(10) \\ \mathrm{C}(35)-\mathrm{Si}(2)-\mathrm{N}(2)-\mathrm{C}(16) & -52.67(11) \\ \mathrm{C}(31)-\mathrm{Si}(2)-\mathrm{N}(2)-\mathrm{C}(16) & 77.40(12) \\ \mathrm{C}(5)-\mathrm{Si}(2)-\mathrm{N}(2)-\mathrm{C}(6) & -0.34(9) \\ \mathrm{C}(35)-\mathrm{Si}(2)-\mathrm{N}(2)-\mathrm{C}(6) & 117.08(8) \\ \mathrm{C}(31)-\mathrm{Si}(2)-\mathrm{N}(2)-\mathrm{C}(6) & -112.84(9) \\ \mathrm{N}(1)-\mathrm{Si}(1)-\mathrm{C}(1)-\mathrm{C}(14) & -156.45(11) \\ \mathrm{C}(23)-\mathrm{Si}(1)-\mathrm{C}(1)-\mathrm{C}(14) & -41.90(13) \\ \mathrm{C}(27)-\mathrm{Si}(1)-\mathrm{C}(1)-\mathrm{C}(14) & 87.27(12) \\ \mathrm{N}(1)-\mathrm{Si}(1)-\mathrm{C}(1)-\mathrm{C}(2) & -29.17(8) \\ \mathrm{C}(23)-\mathrm{Si}(1)-\mathrm{C}(1)-\mathrm{C}(2) & 85.37(9) \\ \mathrm{C}(27)-\mathrm{Si}(1)-\mathrm{C}(1)-\mathrm{C}(2) & -145.45(8) \\ \mathrm{C}(14)-\mathrm{C}(1)-\mathrm{C}(2)-\mathrm{C}(3) & 161.66(11) \\ \mathrm{Si}(1)-\mathrm{C}(1)-\mathrm{C}(2)-\mathrm{C}(3) & 27.77(12) \\ \mathrm{C}(8)-\mathrm{N}(1)-\mathrm{C}(3)-\mathrm{C}(4) & 45.64(14) \\ \mathrm{Si}(1)-\mathrm{N}(1)-\mathrm{C}(3)-\mathrm{C}(4) & -141.13(8) \\ \mathrm{C}(8)-\mathrm{N}(1)-\mathrm{C}(3)-\mathrm{C}(2) & -17.51(12) \\ \mathrm{Si}(1)-\mathrm{N}(1)-\mathrm{C}(3)-\mathrm{C}(2) & \\ \mathrm{C}(1)-\mathrm{C}(2)-\mathrm{C}(3)-\mathrm{N}(1) & \\ & \\ & \end{array}$


$\mathrm{C}(1)-\mathrm{C}(2)-\mathrm{C}(3)-\mathrm{C}(4)$

$\mathrm{N}(1)-\mathrm{C}(3)-\mathrm{C}(4)-\mathrm{C}(6)$

$\mathrm{C}(2)-\mathrm{C}(3)-\mathrm{C}(4)-\mathrm{C}(6)$

$\mathrm{N}(1)-\mathrm{C}(3)-\mathrm{C}(4)-\mathrm{C}(5)$

$\mathrm{C}(2)-\mathrm{C}(3)-\mathrm{C}(4)-\mathrm{C}(5)$

$\mathrm{C}(3)-\mathrm{C}(4)-\mathrm{C}(5)-\mathrm{C}(15)$

$\mathrm{C}(6)-\mathrm{C}(4)-\mathrm{C}(5)-\mathrm{C}(15)$

$\mathrm{C}(3)-\mathrm{C}(4)-\mathrm{C}(5)-\mathrm{Si}(2)$

$\mathrm{C}(6)-\mathrm{C}(4)-\mathrm{C}(5)-\mathrm{Si}(2)$

$\mathrm{N}(2)-\mathrm{Si}(2)-\mathrm{C}(5)-\mathrm{C}(15)$

$\mathrm{C}(35)-\mathrm{Si}(2)-\mathrm{C}(5)-\mathrm{C}(15)$

C(31)-Si(2)-C(5)-C(15)

$\mathrm{N}(2)-\mathrm{Si}(2)-\mathrm{C}(5)-\mathrm{C}(4)$

$\mathrm{C}(35)-\mathrm{Si}(2)-\mathrm{C}(5)-\mathrm{C}(4)$

$\mathrm{C}(31)-\mathrm{Si}(2)-\mathrm{C}(5)-\mathrm{C}(4)$

$\mathrm{C}(16)-\mathrm{N}(2)-\mathrm{C}(6)-\mathrm{C}(7)$

$\mathrm{Si}(2)-\mathrm{N}(2)-\mathrm{C}(6)-\mathrm{C}(7)$

$\mathrm{C}(16)-\mathrm{N}(2)-\mathrm{C}(6)-\mathrm{C}(4)$

$\mathrm{Si}(2)-\mathrm{N}(2)-\mathrm{C}(6)-\mathrm{C}(4)$

$\mathrm{C}(3)-\mathrm{C}(4)-\mathrm{C}(6)-\mathrm{N}(2)$

$\mathrm{C}(5)-\mathrm{C}(4)-\mathrm{C}(6)-\mathrm{N}(2)$

$\mathrm{C}(3)-\mathrm{C}(4)-\mathrm{C}(6)-\mathrm{C}(7)$

$\mathrm{C}(5)-\mathrm{C}(4)-\mathrm{C}(6)-\mathrm{C}(7)$

$\mathrm{N}(2)-\mathrm{C}(6)-\mathrm{C}(7)-\mathrm{C}(12)$

$\mathrm{C}(4)-\mathrm{C}(6)-\mathrm{C}(7)-\mathrm{C}(12)$

$\mathrm{N}(2)-\mathrm{C}(6)-\mathrm{C}(7)-\mathrm{C}(8)$

$\mathrm{C}(4)-\mathrm{C}(6)-\mathrm{C}(7)-\mathrm{C}(8)$

$\mathrm{C}(12)-\mathrm{C}(7)-\mathrm{C}(8)-\mathrm{C}(9)$

$\mathrm{C}(6)-\mathrm{C}(7)-\mathrm{C}(8)-\mathrm{C}(9)$

$\mathrm{C}(12)-\mathrm{C}(7)-\mathrm{C}(8)-\mathrm{N}(1)$

$\mathrm{C}(6)-\mathrm{C}(7)-\mathrm{C}(8)-\mathrm{N}(1)$

C(3)-N(1)-C(8)-C(9)

$\mathrm{Si}(1)-\mathrm{N}(1)-\mathrm{C}(8)-\mathrm{C}(9)$

$\mathrm{C}(3)-\mathrm{N}(1)-\mathrm{C}(8)-\mathrm{C}(7)$

Si(1)-N(1)-C(8)-C(7)

$\mathrm{C}(7)-\mathrm{C}(8)-\mathrm{C}(9)-\mathrm{C}(10)$

$\mathrm{N}(1)-\mathrm{C}(8)-\mathrm{C}(9)-\mathrm{C}(10)$

C(8)-C(9)-C(10)-C(11)
115.24(12)

$-63.98(12)$

170.14(10)

57.98(14)

$-67.90(14)$

68.38(15)

$-169.56(10)$

$-162.63(9)$

$-40.58(10)$

151.53(11)

39.68(12)

$-88.65(11)$

23.85(8)

$-88.01(9)$

143.67(8)

$-91.15(12)$

98.06(10)

146.70(10)

$-24.09(11)$

172.75(9)

42.89(12)

49.62(12)

$-80.24(11)$

$37.85(15)$

158.45(10)

$-141.80(11)$

$-21.19(15)$

1.00(17)

$-179.36(11)$

$-178.11(11)$

1.53(17)

167.33(11)

$-4.46(17)$

$-13.58(16)$

174.63(9)

$-1.67(19)$

177.45(12)

$0.8(2)$ 
$\mathrm{C}(9)-\mathrm{C}(10)-\mathrm{C}(11)-\mathrm{C}(12)$

$\mathrm{C}(9)-\mathrm{C}(10)-\mathrm{C}(11)-\mathrm{C}(13)$

$\mathrm{C}(10)-\mathrm{C}(11)-\mathrm{C}(12)-\mathrm{C}(7)$

$\mathrm{C}(13)-\mathrm{C}(11)-\mathrm{C}(12)-\mathrm{C}(7)$

$\mathrm{C}(8)-\mathrm{C}(7)-\mathrm{C}(12)-\mathrm{C}(11)$

$\mathrm{C}(6)-\mathrm{C}(7)-\mathrm{C}(12)-\mathrm{C}(11)$

$\mathrm{C}(6)-\mathrm{N}(2)-\mathrm{C}(16)-\mathrm{C}(21)$

$\mathrm{Si}(2)-\mathrm{N}(2)-\mathrm{C}(16)-\mathrm{C}(21)$

$\mathrm{C}(6)-\mathrm{N}(2)-\mathrm{C}(16)-\mathrm{C}(17)$

$\mathrm{Si}(2)-\mathrm{N}(2)-\mathrm{C}(16)-\mathrm{C}(17)$

$\mathrm{C}(21)-\mathrm{C}(16)-\mathrm{C}(17)-\mathrm{C}(18)$

$\mathrm{N}(2)-\mathrm{C}(16)-\mathrm{C}(17)-\mathrm{C}(18)$

$\mathrm{C}(16)-\mathrm{C}(17)-\mathrm{C}(18)-\mathrm{C}(19)$

$\mathrm{C}(17)-\mathrm{C}(18)-\mathrm{C}(19)-\mathrm{C}(20)$

$\mathrm{C}(17)-\mathrm{C}(18)-\mathrm{C}(19)-\mathrm{C}(22)$

$\mathrm{C}(18)-\mathrm{C}(19)-\mathrm{C}(20)-\mathrm{C}(21)$

$\mathrm{C}(22)-\mathrm{C}(19)-\mathrm{C}(20)-\mathrm{C}(21)$

$\mathrm{C}(19)-\mathrm{C}(20)-\mathrm{C}(21)-\mathrm{C}(16)$

$\mathrm{C}(17)-\mathrm{C}(16)-\mathrm{C}(21)-\mathrm{C}(20)$

$\mathrm{N}(2)-\mathrm{C}(16)-\mathrm{C}(21)-\mathrm{C}(20)$

$\mathrm{N}(1)-\mathrm{Si}(1)-\mathrm{C}(23)-\mathrm{C}(24)$

C(1)-Si(1)-C(23)-C(24)

C(27)-Si(1)-C(23)-C(24)

$\mathrm{N}(1)-\mathrm{Si}(1)-\mathrm{C}(23)-\mathrm{C}(25)$

C(1)-Si(1)-C(23)-C(25)

$\mathrm{C}(27)-\mathrm{Si}(1)-\mathrm{C}(23)-\mathrm{C}(25)$

$\mathrm{N}(1)-\mathrm{Si}(1)-\mathrm{C}(23)-\mathrm{C}(26)$

C(1)-Si(1)-C(23)-C(26)

$\mathrm{C}(27)-\mathrm{Si}(1)-\mathrm{C}(23)-\mathrm{C}(26)$

$\mathrm{N}(1)-\mathrm{Si}(1)-\mathrm{C}(27)-\mathrm{C}(29)$

$\mathrm{C}(1)-\mathrm{Si}(1)-\mathrm{C}(27)-\mathrm{C}(29)$

$\mathrm{C}(23)-\operatorname{Si}(1)-\mathrm{C}(27)-\mathrm{C}(29)$

$\mathrm{N}(1)-\mathrm{Si}(1)-\mathrm{C}(27)-\mathrm{C}(28)$

C(1)-Si(1)-C(27)-C(28)

$\mathrm{C}(23)-\mathrm{Si}(1)-\mathrm{C}(27)-\mathrm{C}(28)$

$\mathrm{N}(1)-\mathrm{Si}(1)-\mathrm{C}(27)-\mathrm{C}(30)$

C(1)-Si(1)-C(27)-C(30)

C(23)-Si(1)-C(27)-C(30)
0.77(19)

$-179.65(13)$

$-1.45(18)$

178.97(12)

$0.56(18)$

$-179.10(11)$

141.34(12)

$-49.51(16)$

$-40.43(16)$

128.72(11)

1.60(19)

$-176.68(12)$

$0.4(2)$

$-1.4(2)$

178.41(15)

$0.3(2)$

$-179.54(14)$

$1.8(2)$

$-2.72(19)$

175.61(12)

$-61.33(11)$

$-163.73(10)$

70.62(11)

176.70(10)

$74.29(12)$

$-51.36(13)$

$57.50(11)$

$-44.90(11)$

$-170.55(9)$

$-28.88(11)$

$72.20(11)$

$-159.58(10)$

94.75(12)

$-164.17(11)$

$-35.95(14)$

$-145.25(9)$

$-44.17(11)$

84.05(11) 


$\begin{array}{lc}\mathrm{N}(2)-\mathrm{Si}(2)-\mathrm{C}(31)-\mathrm{C}(34) & -91.25(11) \\ \mathrm{C}(5)-\mathrm{Si}(2)-\mathrm{C}(31)-\mathrm{C}(34) & 165.16(10) \\ \mathrm{C}(35)-\mathrm{Si}(2)-\mathrm{C}(31)-\mathrm{C}(34) & 36.24(12) \\ \mathrm{N}(2)-\mathrm{Si}(2)-\mathrm{C}(31)-\mathrm{C}(32) & 147.72(10) \\ \mathrm{C}(5)-\mathrm{Si}(2)-\mathrm{C}(31)-\mathrm{C}(32) & 44.14(11) \\ \mathrm{C}(35)-\mathrm{Si}(2)-\mathrm{C}(31)-\mathrm{C}(32) & -84.79(11) \\ \mathrm{N}(2)-\mathrm{Si}(2)-\mathrm{C}(31)-\mathrm{C}(33) & 31.33(12) \\ \mathrm{C}(5)-\mathrm{Si}(2)-\mathrm{C}(31)-\mathrm{C}(33) & -72.25(11) \\ \mathrm{C}(35)-\mathrm{Si}(2)-\mathrm{C}(31)-\mathrm{C}(33) & 158.82(10) \\ \mathrm{N}(2)-\mathrm{Si}(2)-\mathrm{C}(35)-\mathrm{C}(36) & -53.37(11) \\ \mathrm{C}(5)-\mathrm{Si}(2)-\mathrm{C}(35)-\mathrm{C}(36) & 49.19(11) \\ \mathrm{C}(31)-\mathrm{Si}(2)-\mathrm{C}(35)-\mathrm{C}(36) & 174.78(9) \\ \mathrm{N}(2)-\mathrm{Si}(2)-\mathrm{C}(35)-\mathrm{C}(37) & -173.16(9) \\ \mathrm{C}(5)-\mathrm{Si}(2)-\mathrm{C}(35)-\mathrm{C}(37) & -70.61(11) \\ \mathrm{C}(31)-\mathrm{Si}(2)-\mathrm{C}(35)-\mathrm{C}(37) & 54.98(11) \\ \mathrm{N}(2)-\operatorname{Si}(2)-\mathrm{C}(35)-\mathrm{C}(38) & 64.71(10) \\ \mathrm{C}(5)-\mathrm{Si}(2)-\mathrm{C}(35)-\mathrm{C}(38) & 167.26(9) \\ \mathrm{C}(31)-\mathrm{Si}(2)-\mathrm{C}(35)-\mathrm{C}(38) & -67.15(11) \\ & \end{array}$




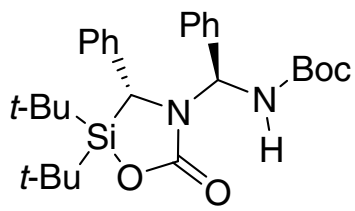

13

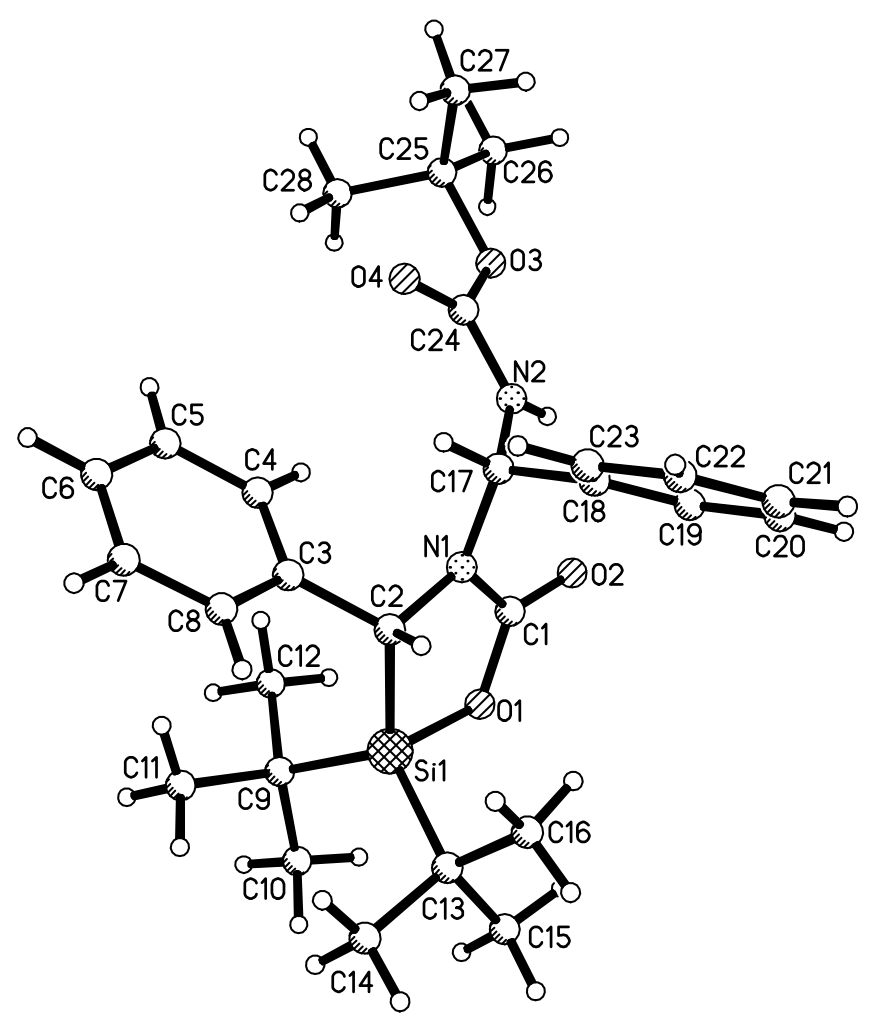

Carbamic Acid Silyl Ester 13. See text above

X-ray Data Collection, Structure Solution and Refinement for 13.

A colorless crystal of approximate dimensions $0.13 \times 0.24 \times 0.26 \mathrm{~mm}$ was mounted on a glass fiber and transferred to a Bruker CCD platform diffractometer. The SMART ${ }^{1}$ program package was used to determine the unit-cell parameters and for data collection (40 sec/frame scan time for a hemisphere of diffraction data). The raw frame data was processed using $\mathrm{SAINT}^{2}$ and $\mathrm{SADABS}^{3}$ to yield the reflection data file. Subsequent calculations were carried out using the SHELXTL ${ }^{4}$ program. The diffraction symmetry was $2 / m$ and the systematic absences were consistent with the centrosymmetric monoclinic space group $P 2_{1} / c$ that was later determined to be correct.

The structure was solved by direct methods and refined on $\mathrm{F}^{2}$ by full-matrix least-squares techniques. The analytical scattering factors ${ }^{5}$ for neutral atoms were used throughout the analysis. Hydrogen atoms were located from a difference-Fourier map and refined $\left(\mathrm{x}, \mathrm{y}, \mathrm{z}\right.$ and $\left.\mathrm{U}_{\mathrm{iso}}\right)$. At convergence, $\mathrm{wR} 2=0.0991$ and Goof $=1.025$ for 475 variables refined against 6054 data $(0.78 \AA), R 1=0.0377$ for those 4644 data with $\mathrm{I}>2.0 \sigma(\mathrm{I})$.

\section{References.}

6. SMART Software Users Guide, Version 5.1, Bruker Analytical X-Ray Systems, Inc.; Madison, WI 1999.

7. SAINT Software Users Guide, Version 6.0, Bruker Analytical X-Ray Systems, Inc.; Madison, WI 1999.

8. Sheldrick, G. M. SADABS, Version 2.10, Bruker Analytical X-Ray Systems, Inc.; Madison, WI 2002.

9. Sheldrick, G. M. SHELXTL Version 6.12, Bruker Analytical X-Ray Systems, Inc.; Madison, WI 2001. 
10. International Tables for X-Ray Crystallography 1992, Vol. C., Dordrecht: Kluwer Academic Publishers.

Definitions:

$\mathrm{wR} 2=\left[\Sigma\left[\mathrm{w}\left(\mathrm{F}_{\mathrm{o}}^{2}-\mathrm{F}_{\mathrm{c}}^{2}\right)^{2}\right] / \Sigma\left[\mathrm{w}\left(\mathrm{F}_{\mathrm{o}}^{2}\right)^{2}\right]\right]^{1 / 2}$

$\mathrm{R} 1=\Sigma\left\|\mathrm{F}_{\mathrm{o}}|-| \mathrm{F}_{\mathrm{c}}\right\| / \Sigma\left|\mathrm{F}_{\mathrm{o}}\right|$

Goof $=\mathrm{S}=\left[\Sigma\left[\mathrm{w}\left(\mathrm{F}_{\mathrm{o}}^{2}-\mathrm{F}_{\mathrm{c}}{ }^{2}\right)^{2}\right] /(\mathrm{n}-\mathrm{p})\right]^{1 / 2}$ where $\mathrm{n}$ is the number of reflections and $\mathrm{p}$ is the total number of parameters refined.

The thermal ellipsoid plot is shown at the $50 \%$ probability level.

Table 1. Crystal data and structure refinement for $\mathbf{1 3 .}$

Identification code

Empirical formula

Formula weight

Temperature

Wavelength

Crystal system

Space group

Unit cell dimensions

Volume

$\mathrm{Z}$

Density (calculated)

Absorption coefficient

$\mathrm{F}(000)$

Crystal color

Crystal size

Theta range for data collection

Index ranges

Reflections collected

Independent reflections

Completeness to theta $=27.10^{\circ}$

Absorption correction

Max. and min. transmission

Refinement method

Data / restraints / parameters

\section{3 (Zulimar Nevárez)}

$\mathrm{C}_{28} \mathrm{H}_{40} \mathrm{~N}_{2} \mathrm{O}_{4} \mathrm{Si}$

496.71

163(2) K

$0.71073 \AA$

Monoclinic

$P 2{ }_{1} / c$

$a=10.9029(9) \AA \quad \alpha=90^{\circ}$.

$\mathrm{b}=21.2730(18) \AA \quad \beta=105.929(2)^{\circ}$.

$\mathrm{c}=12.3464(10) \AA \quad \gamma=90^{\circ}$.

2753.6(4) $\AA^{3}$

4

$1.198 \mathrm{Mg} / \mathrm{m}^{3}$

$0.120 \mathrm{~mm}^{-1}$

1072

colorless

$0.26 \times 0.24 \times 0.13 \mathrm{~mm}^{3}$

1.91 to $27.10^{\circ}$

$-11 \leq h \leq 13,-25 \leq k \leq 27,-15 \leq l \leq 15$

20197

$6054[\mathrm{R}(\mathrm{int})=0.0363]$

$99.9 \%$

Semi-empirical from equivalents

0.9846 and 0.9695

Full-matrix least-squares on $\mathrm{F}^{2}$

6054 / 0 / 475 
Goodness-of-fit on $\mathrm{F}^{2}$

Final $\mathrm{R}$ indices [I $>2 \operatorname{sigma}(\mathrm{I})=4644$ data]

$\mathrm{R}$ indices (all data; $0.78 \AA$ )

Largest diff. peak and hole
1.025

$\mathrm{R} 1=0.0377, \mathrm{wR} 2=0.0875$

$\mathrm{R} 1=0.0576, \mathrm{wR} 2=0.0991$

0.322 and -0.304 e. $\AA^{-3}$

Table 2. Atomic coordinates ( x 104) and equivalent isotropic displacement parameters $\left(\AA^{2} \times 10^{3}\right)$ for 13. $U(e q)$ is defined as one third of the trace of the orthogonalized $U^{i j}$ tensor.

\begin{tabular}{|c|c|c|c|c|}
\hline & $\mathrm{X}$ & $\mathrm{y}$ & $\mathrm{z}$ & $\mathrm{U}(\mathrm{eq})$ \\
\hline $\operatorname{Si}(1)$ & $703(1)$ & 1972(1) & $1274(1)$ & $18(1)$ \\
\hline $\mathrm{O}(1)$ & $1264(1)$ & $2032(1)$ & $2698(1)$ & $23(1)$ \\
\hline $\mathrm{O}(2)$ & $3053(1)$ & 1989(1) & $4123(1)$ & $24(1)$ \\
\hline $\mathrm{O}(3)$ & $6309(1)$ & $694(1)$ & $4554(1)$ & $27(1)$ \\
\hline $\mathrm{O}(4)$ & 6191(1) & $804(1)$ & $2688(1)$ & $33(1)$ \\
\hline $\mathrm{N}(1)$ & $3140(1)$ & $1818(1)$ & $2312(1)$ & 19(1) \\
\hline $\mathrm{N}(2)$ & $5103(1)$ & $1444(1)$ & $3572(1)$ & $22(1)$ \\
\hline $\mathrm{C}(1)$ & $2537(1)$ & 1949(1) & $3118(1)$ & 19(1) \\
\hline $\mathrm{C}(2)$ & $2395(1)$ & $1847(1)$ & $1117(1)$ & $18(1)$ \\
\hline$C(3)$ & $2642(1)$ & 1291(1) & $436(1)$ & $18(1)$ \\
\hline $\mathrm{C}(4)$ & $3171(2)$ & $729(1)$ & $929(1)$ & $22(1)$ \\
\hline$C(5)$ & $3409(2)$ & $242(1)$ & $267(1)$ & $26(1)$ \\
\hline$C(6)$ & $3110(2)$ & $300(1)$ & $-893(1)$ & $28(1)$ \\
\hline$C(7)$ & $2565(2)$ & $855(1)$ & $-1389(1)$ & $28(1)$ \\
\hline $\mathrm{C}(8)$ & $2353(2)$ & $1350(1)$ & $-730(1)$ & $23(1)$ \\
\hline $\mathrm{C}(9)$ & $-410(2)$ & $1275(1)$ & 974(1) & $25(1)$ \\
\hline$C(10)$ & $-1679(2)$ & $1445(1)$ & $1223(2)$ & $32(1)$ \\
\hline $\mathrm{C}(11)$ & $-684(2)$ & $1044(1)$ & $-250(2)$ & $34(1)$ \\
\hline$C(12)$ & $201(2)$ & $733(1)$ & $1772(2)$ & $35(1)$ \\
\hline$C(13)$ & $16(2)$ & $2769(1)$ & $751(1)$ & $23(1)$ \\
\hline$C(14)$ & $-794(2)$ & $2750(1)$ & $-479(2)$ & $35(1)$ \\
\hline$C(15)$ & $-798(2)$ & $3015(1)$ & $1503(2)$ & $33(1)$ \\
\hline$C(16)$ & $1123(2)$ & $3233(1)$ & $852(2)$ & $31(1)$ \\
\hline$C(17)$ & $4549(1)$ & $1827(1)$ & 2591(1) & $20(1)$ \\
\hline $\mathrm{C}(18)$ & $5079(1)$ & 2491(1) & $2682(1)$ & $20(1)$ \\
\hline$C(19)$ & $5083(2)$ & $2885(1)$ & $3583(1)$ & $26(1)$ \\
\hline$C(20)$ & $5572(2)$ & $3488(1)$ & $3631(2)$ & $31(1)$ \\
\hline
\end{tabular}




$\begin{array}{lrrrr}\mathrm{C}(21) & 6069(2) & 3706(1) & 2783(2) & 31(1) \\ \mathrm{C}(22) & 6067(2) & 3321(1) & 1882(2) & 32(1) \\ \mathrm{C}(23) & 5575(2) & 2715(1) & 1833(1) & 27(1) \\ \mathrm{C}(24) & 5899(1) & 961(1) & 3524(1) & 22(1) \\ \mathrm{C}(25) & 7079(2) & 109(1) & 4702(2) & 31(1) \\ \mathrm{C}(26) & 7328(3) & -19(1) & 5953(2) & 49(1) \\ \mathrm{C}(27) & 8309(2) & 218(1) & 4380(2) & 43(1) \\ \mathrm{C}(28) & 6285(2) & -418(1) & 4024(2) & 33(1)\end{array}$

Table 3. Bond lengths $[\AA]$ and angles $\left[{ }^{\circ}\right]$ for $\mathbf{1 3}$.

\begin{tabular}{ll}
\hline $\mathrm{Si}(1)-\mathrm{O}(1)$ & $1.7008(11)$ \\
$\mathrm{Si}(1)-\mathrm{C}(9)$ & $1.8876(16)$ \\
$\mathrm{Si}(1)-\mathrm{C}(13)$ & $1.8949(16)$ \\
$\mathrm{Si}(1)-\mathrm{C}(2)$ & $1.9252(15)$ \\
$\mathrm{O}(1)-\mathrm{C}(1)$ & $1.3534(18)$ \\
$\mathrm{O}(2)-\mathrm{C}(1)$ & $1.2162(17)$ \\
$\mathrm{O}(3)-\mathrm{C}(24)$ & $1.3517(18)$ \\
$\mathrm{O}(3)-\mathrm{C}(25)$ & $1.4828(18)$ \\
$\mathrm{O}(4)-\mathrm{C}(24)$ & $1.2074(18)$ \\
$\mathrm{N}(1)-\mathrm{C}(1)$ & $1.3635(19)$ \\
$\mathrm{N}(1)-\mathrm{C}(2)$ & $1.4779(18)$ \\
$\mathrm{N}(1)-\mathrm{C}(17)$ & $1.4800(19)$ \\
$\mathrm{N}(2)-\mathrm{C}(24)$ & $1.3571(19)$ \\
$\mathrm{N}(2)-\mathrm{C}(17)$ & $1.4466(19)$ \\
$\mathrm{C}(2)-\mathrm{C}(3)$ & $1.516(2)$ \\
$\mathrm{C}(3)-\mathrm{C}(8)$ & $1.392(2)$ \\
$\mathrm{C}(3)-\mathrm{C}(4)$ & $1.393(2)$ \\
$\mathrm{C}(4)-\mathrm{C}(5)$ & $1.388(2)$ \\
$\mathrm{C}(5)-\mathrm{C}(6)$ & $1.383(2)$ \\
$\mathrm{C}(6)-\mathrm{C}(7)$ & $1.386(2)$ \\
$\mathrm{C}(7)-\mathrm{C}(8)$ & $1.388(2)$ \\
$\mathrm{C}(9)-\mathrm{C}(11)$ & $1.537(2)$ \\
$\mathrm{C}(9)-\mathrm{C}(10)$ & $1.540(2)$ \\
$\mathrm{C}(9)-\mathrm{C}(12)$ & $1.543(2)$ \\
$\mathrm{C}(13)-\mathrm{C}(14)$ & $1.533(2)$ \\
& \\
&
\end{tabular}




\begin{tabular}{|c|c|}
\hline$C(13)-C(16)$ & $1.537(2)$ \\
\hline$C(13)-C(15)$ & $1.542(2)$ \\
\hline $\mathrm{C}(17)-\mathrm{C}(18)$ & $1.519(2)$ \\
\hline $\mathrm{C}(18)-\mathrm{C}(23)$ & $1.389(2)$ \\
\hline $\mathrm{C}(18)-\mathrm{C}(19)$ & $1.391(2)$ \\
\hline$C(19)-C(20)$ & $1.384(2)$ \\
\hline$C(20)-C(21)$ & $1.384(3)$ \\
\hline$C(21)-C(22)$ & $1.381(3)$ \\
\hline$C(22)-C(23)$ & $1.392(2)$ \\
\hline$C(25)-C(27)$ & $1.517(3)$ \\
\hline$C(25)-C(26)$ & $1.518(3)$ \\
\hline$C(25)-C(28)$ & $1.519(2)$ \\
\hline $\mathrm{O}(1)-\mathrm{Si}(1)-\mathrm{C}(9)$ & $106.97(6)$ \\
\hline $\mathrm{O}(1)-\mathrm{Si}(1)-\mathrm{C}(13)$ & $106.31(6)$ \\
\hline $\mathrm{C}(9)-\mathrm{Si}(1)-\mathrm{C}(13)$ & $117.61(7)$ \\
\hline $\mathrm{O}(1)-\mathrm{Si}(1)-\mathrm{C}(2)$ & $91.87(6)$ \\
\hline $\mathrm{C}(9)-\mathrm{Si}(1)-\mathrm{C}(2)$ & $117.04(7)$ \\
\hline $\mathrm{C}(13)-\mathrm{Si}(1)-\mathrm{C}(2)$ & $112.87(7)$ \\
\hline $\mathrm{C}(1)-\mathrm{O}(1)-\mathrm{Si}(1)$ & $115.19(9)$ \\
\hline $\mathrm{C}(24)-\mathrm{O}(3)-\mathrm{C}(25)$ & $119.87(12)$ \\
\hline $\mathrm{C}(1)-\mathrm{N}(1)-\mathrm{C}(2)$ & $118.52(12)$ \\
\hline $\mathrm{C}(1)-\mathrm{N}(1)-\mathrm{C}(17)$ & $120.31(12)$ \\
\hline $\mathrm{C}(2)-\mathrm{N}(1)-\mathrm{C}(17)$ & $118.87(11)$ \\
\hline $\mathrm{C}(24)-\mathrm{N}(2)-\mathrm{C}(17)$ & $120.99(13)$ \\
\hline $\mathrm{O}(2)-\mathrm{C}(1)-\mathrm{O}(1)$ & $121.34(13)$ \\
\hline $\mathrm{O}(2)-\mathrm{C}(1)-\mathrm{N}(1)$ & $125.30(14)$ \\
\hline $\mathrm{O}(1)-\mathrm{C}(1)-\mathrm{N}(1)$ & $113.36(12)$ \\
\hline $\mathrm{N}(1)-\mathrm{C}(2)-\mathrm{C}(3)$ & $112.80(12)$ \\
\hline $\mathrm{N}(1)-\mathrm{C}(2)-\mathrm{Si}(1)$ & $100.59(9)$ \\
\hline $\mathrm{C}(3)-\mathrm{C}(2)-\mathrm{Si}(1)$ & $118.93(10)$ \\
\hline $\mathrm{C}(8)-\mathrm{C}(3)-\mathrm{C}(4)$ & $118.47(14)$ \\
\hline $\mathrm{C}(8)-\mathrm{C}(3)-\mathrm{C}(2)$ & $118.63(13)$ \\
\hline $\mathrm{C}(4)-\mathrm{C}(3)-\mathrm{C}(2)$ & $122.89(13)$ \\
\hline$C(5)-C(4)-C(3)$ & $120.41(15)$ \\
\hline$C(6)-C(5)-C(4)$ & $120.90(15)$ \\
\hline$C(5)-C(6)-C(7)$ & $118.95(15)$ \\
\hline$C(6)-C(7)-C(8)$ & $120.40(15)$ \\
\hline$C(7)-C(8)-C(3)$ & $120.84(15)$ \\
\hline
\end{tabular}




$\begin{array}{ll}\mathrm{C}(11)-\mathrm{C}(9)-\mathrm{C}(10) & 109.18(14) \\ \mathrm{C}(11)-\mathrm{C}(9)-\mathrm{C}(12) & 108.85(15) \\ \mathrm{C}(10)-\mathrm{C}(9)-\mathrm{C}(12) & 107.59(14) \\ \mathrm{C}(11)-\mathrm{C}(9)-\mathrm{Si}(1) & 112.62(11) \\ \mathrm{C}(10)-\mathrm{C}(9)-\mathrm{Si}(1) & 109.88(11) \\ \mathrm{C}(12)-\mathrm{C}(9)-\mathrm{Si}(1) & 108.59(11) \\ \mathrm{C}(14)-\mathrm{C}(13)-\mathrm{C}(16) & 108.88(14) \\ \mathrm{C}(14)-\mathrm{C}(13)-\mathrm{C}(15) & 109.55(15) \\ \mathrm{C}(16)-\mathrm{C}(13)-\mathrm{C}(15) & 107.56(14) \\ \mathrm{C}(14)-\mathrm{C}(13)-\mathrm{Si}(1) & 112.36(12) \\ \mathrm{C}(16)-\mathrm{C}(13)-\mathrm{Si}(1) & 108.64(11) \\ \mathrm{C}(15)-\mathrm{C}(13)-\mathrm{Si}(1) & 109.74(11) \\ \mathrm{N}(2)-\mathrm{C}(17)-\mathrm{N}(1) & 110.84(12) \\ \mathrm{N}(2)-\mathrm{C}(17)-\mathrm{C}(18) & 113.53(12) \\ \mathrm{N}(1)-\mathrm{C}(17)-\mathrm{C}(18) & 112.28(12) \\ \mathrm{C}(23)-\mathrm{C}(18)-\mathrm{C}(19) & 118.72(15) \\ \mathrm{C}(23)-\mathrm{C}(18)-\mathrm{C}(17) & 118.80(14) \\ \mathrm{C}(19)-\mathrm{C}(18)-\mathrm{C}(17) & 122.48(14) \\ \mathrm{C}(20)-\mathrm{C}(19)-\mathrm{C}(18) & 120.59(16) \\ \mathrm{C}(21)-\mathrm{C}(20)-\mathrm{C}(19) & 120.31(17) \\ \mathrm{C}(22)-\mathrm{C}(21)-\mathrm{C}(20) & 119.75(16) \\ \mathrm{C}(21)-\mathrm{C}(22)-\mathrm{C}(23) & 119.99(16) \\ \mathrm{C}(18)-\mathrm{C}(23)-\mathrm{C}(22) & 120.65(16) \\ \mathrm{O}(4)-\mathrm{C}(24)-\mathrm{O}(3) & 126.27(14) \\ \mathrm{O}(4)-\mathrm{C}(24)-\mathrm{N}(2) & 124.62(15) \\ \mathrm{O}(3)-\mathrm{C}(24)-\mathrm{N}(2) & 109.10(13) \\ \mathrm{O}(3)-\mathrm{C}(25)-\mathrm{C}(27) & 110.51(14) \\ \mathrm{O}(3)-\mathrm{C}(25)-\mathrm{C}(26) & 102.30(14) \\ \mathrm{C}(27)-\mathrm{C}(25)-\mathrm{C}(26) & 111.70(18) \\ \mathrm{O}(3)-\mathrm{C}(25)-\mathrm{C}(28) & 109.48(13) \\ \mathrm{C}(27)-\mathrm{C}(25)-\mathrm{C}(28) & 112.28(16) \\ \mathrm{C}(26)-\mathrm{C}(25)-\mathrm{C}(28) & 110.13(17)\end{array}$


Table 4. Anisotropic displacement parameters $\left(\AA^{2} \times 10^{3}\right)$ for 13. The anisotropic displacement factor exponent takes the form: $-2 \pi^{2}\left[\mathrm{~h}^{2} \mathrm{a}^{* 2} \mathrm{U}^{11}+\ldots+2 \mathrm{~h} \mathrm{k} \mathrm{a}^{*} \mathrm{~b}^{*} \mathrm{U}^{12}\right]$

\begin{tabular}{|c|c|c|c|c|c|c|}
\hline & $\mathrm{U}^{11}$ & $\mathrm{U}^{22}$ & $\mathrm{U}^{33}$ & $\mathrm{U}^{23}$ & $\mathrm{U}^{13}$ & $\mathrm{U}^{12}$ \\
\hline $\operatorname{Si}(1)$ & $18(1)$ & $17(1)$ & $21(1)$ & $1(1)$ & $6(1)$ & $0(1)$ \\
\hline $\mathrm{O}(1)$ & $17(1)$ & $30(1)$ & $22(1)$ & $0(1)$ & $8(1)$ & $2(1)$ \\
\hline $\mathrm{O}(2)$ & $23(1)$ & $29(1)$ & $20(1)$ & $-2(1)$ & $7(1)$ & $1(1)$ \\
\hline $\mathrm{O}(3)$ & $27(1)$ & $22(1)$ & $29(1)$ & $-1(1)$ & $3(1)$ & $10(1)$ \\
\hline $\mathrm{O}(4)$ & $34(1)$ & $34(1)$ & $33(1)$ & $-2(1)$ & $15(1)$ & $12(1)$ \\
\hline $\mathrm{N}(1)$ & $16(1)$ & $21(1)$ & $20(1)$ & $-3(1)$ & $6(1)$ & $0(1)$ \\
\hline $\mathrm{N}(2)$ & $23(1)$ & $21(1)$ & $22(1)$ & $-1(1)$ & $8(1)$ & $7(1)$ \\
\hline $\mathrm{C}(1)$ & $20(1)$ & $17(1)$ & $22(1)$ & $0(1)$ & $7(1)$ & $-1(1)$ \\
\hline $\mathrm{C}(2)$ & $19(1)$ & $16(1)$ & $19(1)$ & $0(1)$ & $7(1)$ & $0(1)$ \\
\hline $\mathrm{C}(3)$ & $17(1)$ & $17(1)$ & $23(1)$ & $-3(1)$ & $7(1)$ & $-3(1)$ \\
\hline $\mathrm{C}(4)$ & $23(1)$ & $19(1)$ & $24(1)$ & $1(1)$ & $8(1)$ & $-1(1)$ \\
\hline $\mathrm{C}(5)$ & $29(1)$ & $15(1)$ & $37(1)$ & $0(1)$ & 11(1) & $0(1)$ \\
\hline$C(6)$ & $35(1)$ & $19(1)$ & $34(1)$ & $-9(1)$ & $17(1)$ & $-5(1)$ \\
\hline$C(7)$ & $36(1)$ & $27(1)$ & $23(1)$ & $-4(1)$ & $13(1)$ & $-4(1)$ \\
\hline $\mathrm{C}(8)$ & $27(1)$ & $20(1)$ & $25(1)$ & $1(1)$ & $9(1)$ & $0(1)$ \\
\hline $\mathrm{C}(9)$ & $21(1)$ & $23(1)$ & $30(1)$ & $3(1)$ & $7(1)$ & $-3(1)$ \\
\hline$C(10)$ & $22(1)$ & $32(1)$ & $42(1)$ & $3(1)$ & $10(1)$ & $-5(1)$ \\
\hline $\mathrm{C}(11)$ & $30(1)$ & $32(1)$ & $39(1)$ & $-9(1)$ & $10(1)$ & $-10(1)$ \\
\hline$C(12)$ & $28(1)$ & $27(1)$ & $48(1)$ & $12(1)$ & $9(1)$ & $-2(1)$ \\
\hline$C(13)$ & $25(1)$ & $19(1)$ & $27(1)$ & $4(1)$ & $12(1)$ & $4(1)$ \\
\hline$C(14)$ & $40(1)$ & $33(1)$ & $31(1)$ & $9(1)$ & $6(1)$ & $7(1)$ \\
\hline$C(15)$ & $37(1)$ & $27(1)$ & $44(1)$ & $5(1)$ & $23(1)$ & $10(1)$ \\
\hline$C(16)$ & $35(1)$ & $18(1)$ & $44(1)$ & $1(1)$ & $20(1)$ & $1(1)$ \\
\hline $\mathrm{C}(17)$ & $18(1)$ & $22(1)$ & $20(1)$ & $-3(1)$ & $6(1)$ & $2(1)$ \\
\hline $\mathrm{C}(18)$ & $13(1)$ & $22(1)$ & $23(1)$ & $1(1)$ & $1(1)$ & $3(1)$ \\
\hline$C(19)$ & $28(1)$ & $24(1)$ & $28(1)$ & $-2(1)$ & $9(1)$ & $-4(1)$ \\
\hline$C(20)$ & $29(1)$ & $25(1)$ & $35(1)$ & $-4(1)$ & $4(1)$ & $-2(1)$ \\
\hline $\mathrm{C}(21)$ & $23(1)$ & $23(1)$ & $41(1)$ & $6(1)$ & $1(1)$ & $-2(1)$ \\
\hline$C(22)$ & $27(1)$ & $34(1)$ & $36(1)$ & 11(1) & $11(1)$ & $0(1)$ \\
\hline$C(23)$ & $26(1)$ & $29(1)$ & $28(1)$ & $0(1)$ & $9(1)$ & $2(1)$ \\
\hline$C(24)$ & $18(1)$ & $19(1)$ & $29(1)$ & $-4(1)$ & $5(1)$ & $0(1)$ \\
\hline$C(25)$ & $24(1)$ & $21(1)$ & $41(1)$ & $-3(1)$ & $1(1)$ & $10(1)$ \\
\hline$C(26)$ & $58(2)$ & $38(1)$ & $41(1)$ & $1(1)$ & $-6(1)$ & $24(1)$ \\
\hline
\end{tabular}




$\begin{array}{lllllll}\mathrm{C}(27) & 19(1) & 30(1) & 75(2) & -11(1) & 3(1) & 5(1) \\ \mathrm{C}(28) & 27(1) & 23(1) & 47(1) & -4(1) & 9(1) & 0(1)\end{array}$

Table 5. Hydrogen coordinates ( $\left.x 10^{4}\right)$ and isotropic displacement parameters $\left(\AA^{2} \times 10^{3}\right)$ for 13.

\begin{tabular}{|c|c|c|c|c|}
\hline & $\mathrm{x}$ & $\mathrm{y}$ & $\mathrm{z}$ & $\mathrm{U}(\mathrm{eq})$ \\
\hline $\mathrm{H}(2)$ & $4889(16)$ & $1516(8)$ & $4182(15)$ & $23(4)$ \\
\hline $\mathrm{H}(2 \mathrm{~A})$ & $2638(15)$ & $2231(8)$ & 781(13) & $15(4)$ \\
\hline $\mathrm{H}(4 \mathrm{~A})$ & $3364(16)$ & $689(8)$ & $1732(15)$ & $25(4)$ \\
\hline $\mathrm{H}(5 \mathrm{~A})$ & $3806(17)$ & $-128(9)$ & $612(15)$ & $31(5)$ \\
\hline $\mathrm{H}(6 \mathrm{~A})$ & $3293(17)$ & $-43(9)$ & $-1370(15)$ & $32(5)$ \\
\hline $\mathrm{H}(7 \mathrm{~A})$ & $2373(17)$ & $902(9)$ & $-2196(16)$ & $35(5)$ \\
\hline $\mathrm{H}(8 \mathrm{~A})$ & $1988(16)$ & 1733(9) & $-1080(14)$ & $28(5)$ \\
\hline $\mathrm{H}(10 \mathrm{~A})$ & $-2177(17)$ & 1773(9) & $683(15)$ & $29(5)$ \\
\hline $\mathrm{H}(10 \mathrm{~B})$ & $-1565(18)$ & 1612(9) & $2012(17)$ & $37(5)$ \\
\hline $\mathrm{H}(10 \mathrm{C})$ & $-2216(19)$ & $1055(10)$ & $1137(16)$ & $41(5)$ \\
\hline $\mathrm{H}(11 \mathrm{~A})$ & $-1091(19)$ & $1385(10)$ & $-831(17)$ & $46(6)$ \\
\hline $\mathrm{H}(11 \mathrm{~B})$ & $-1307(19)$ & $677(10)$ & $-366(16)$ & $44(6)$ \\
\hline $\mathrm{H}(11 \mathrm{C})$ & $70(20)$ & $890(10)$ & $-417(17)$ & $45(6)$ \\
\hline $\mathrm{H}(12 \mathrm{~A})$ & $329(19)$ & $855(10)$ & 2578(18) & $47(6)$ \\
\hline $\mathrm{H}(12 \mathrm{~B})$ & $-370(20)$ & $350(10)$ & $1592(16)$ & $45(6)$ \\
\hline $\mathrm{H}(12 \mathrm{C})$ & $1040(20)$ & $601(10)$ & $1672(17)$ & $48(6)$ \\
\hline $\mathrm{H}(14 \mathrm{~A})$ & $-1128(19)$ & $3182(10)$ & $-725(16)$ & $42(5)$ \\
\hline $\mathrm{H}(14 \mathrm{~B})$ & $-309(19)$ & 2592(9) & $-992(17)$ & $40(5)$ \\
\hline $\mathrm{H}(14 \mathrm{C})$ & $-1540(20)$ & $2479(10)$ & $-612(16)$ & $39(5)$ \\
\hline $\mathrm{H}(15 \mathrm{~A})$ & $-270(20)$ & $3064(9)$ & $2316(18)$ & $43(6)$ \\
\hline $\mathrm{H}(15 \mathrm{~B})$ & $-1144(19)$ & $3430(10)$ & $1220(17)$ & $45(6)$ \\
\hline $\mathrm{H}(15 \mathrm{C})$ & $-1520(20)$ & $2748(10)$ & 1491(17) & $47(6)$ \\
\hline $\mathrm{H}(16 \mathrm{~A})$ & $1586(17)$ & $3159(8)$ & $289(15)$ & $29(5)$ \\
\hline $\mathrm{H}(16 \mathrm{~B})$ & $1723(18)$ & $3216(8)$ & $1588(16)$ & $30(5)$ \\
\hline $\mathrm{H}(16 \mathrm{C})$ & $796(18)$ & $3665(10)$ & $728(15)$ & $37(5)$ \\
\hline $\mathrm{H}(17 \mathrm{~A})$ & $4762(18)$ & 1631(9) & $1944(16)$ & 36 \\
\hline $\mathrm{H}(19 \mathrm{~A})$ & $4737(16)$ & 2741(8) & $4178(15)$ & $27(5)$ \\
\hline
\end{tabular}




$\begin{array}{lllll}\mathrm{H}(20 \mathrm{~A}) & 5575(18) & 3759(10) & 4242(17) & 41(5) \\ \mathrm{H}(21 \mathrm{~A}) & 6435(18) & 4131(10) & 2826(15) & 37(5) \\ \mathrm{H}(22 \mathrm{~A}) & 6405(18) & 3463(9) & 1290(16) & 35(5) \\ \mathrm{H}(23 \mathrm{~A}) & 5583(17) & 2434(9) & 1221(15) & 31(5) \\ \mathrm{H}(26 \mathrm{~A}) & 7830(20) & 322(11) & 6377(19) & 57(7) \\ \mathrm{H}(26 \mathrm{~B}) & 6470(30) & -52(12) & 6180(20) & 69(8) \\ \mathrm{H}(26 \mathrm{C}) & 7800(20) & -416(11) & 6103(18) & 56(6) \\ \mathrm{H}(27 \mathrm{~A}) & 8750(20) & 570(12) & 4820(19) & 60(7) \\ \mathrm{H}(27 \mathrm{~B}) & 8180(20) & 293(10) & 3560(20) & 51(7) \\ \mathrm{H}(27 \mathrm{C}) & 8860(20) & -144(12) & 4642(19) & 61(7) \\ \mathrm{H}(28 \mathrm{~A}) & 6063(18) & -336(9) & 3201(18) & 41(6) \\ \mathrm{H}(28 \mathrm{~B}) & 5510(20) & -481(9) & 4280(16) & 40(5) \\ \mathrm{H}(28 \mathrm{C}) & 6740(20) & -814(11) & 4197(17) & 49(6)\end{array}$

Table 6. Torsion angles $\left[{ }^{\circ}\right]$ for 13.

\begin{tabular}{lc}
\hline $\mathrm{C}(9)-\mathrm{Si}(1)-\mathrm{O}(1)-\mathrm{C}(1)$ & $116.48(11)$ \\
$\mathrm{C}(13)-\mathrm{Si}(1)-\mathrm{O}(1)-\mathrm{C}(1)$ & $-117.06(11)$ \\
$\mathrm{C}(2)-\mathrm{Si}(1)-\mathrm{O}(1)-\mathrm{C}(1)$ & $-2.57(11)$ \\
$\mathrm{Si}(1)-\mathrm{O}(1)-\mathrm{C}(1)-\mathrm{O}(2)$ & $179.15(11)$ \\
$\mathrm{Si}(1)-\mathrm{O}(1)-\mathrm{C}(1)-\mathrm{N}(1)$ & $-1.37(16)$ \\
$\mathrm{C}(2)-\mathrm{N}(1)-\mathrm{C}(1)-\mathrm{O}(2)$ & $-174.19(14)$ \\
$\mathrm{C}(17)-\mathrm{N}(1)-\mathrm{C}(1)-\mathrm{O}(2)$ & $-11.6(2)$ \\
$\mathrm{C}(2)-\mathrm{N}(1)-\mathrm{C}(1)-\mathrm{O}(1)$ & $6.35(18)$ \\
$\mathrm{C}(17)-\mathrm{N}(1)-\mathrm{C}(1)-\mathrm{O}(1)$ & $168.93(12)$ \\
$\mathrm{C}(1)-\mathrm{N}(1)-\mathrm{C}(2)-\mathrm{C}(3)$ & $-135.12(13)$ \\
$\mathrm{C}(17)-\mathrm{N}(1)-\mathrm{C}(2)-\mathrm{C}(3)$ & $62.05(16)$ \\
$\mathrm{C}(1)-\mathrm{N}(1)-\mathrm{C}(2)-\mathrm{Si}(1)$ & $-7.35(14)$ \\
$\mathrm{C}(17)-\mathrm{N}(1)-\mathrm{C}(2)-\mathrm{Si}(1)$ & $-170.18(10)$ \\
$\mathrm{O}(1)-\mathrm{Si}(1)-\mathrm{C}(2)-\mathrm{N}(1)$ & $5.15(9)$ \\
$\mathrm{C}(9)-\mathrm{Si}(1)-\mathrm{C}(2)-\mathrm{N}(1)$ & $-105.00(10)$ \\
$\mathrm{C}(13)-\mathrm{Si}(1)-\mathrm{C}(2)-\mathrm{N}(1)$ & $113.73(10)$ \\
$\mathrm{O}(1)-\mathrm{Si}(1)-\mathrm{C}(2)-\mathrm{C}(3)$ & $128.77(11)$ \\
$\mathrm{C}(9)-\mathrm{Si}(1)-\mathrm{C}(2)-\mathrm{C}(3)$ & $18.62(14)$ \\
$\mathrm{C}(13)-\mathrm{Si}(1)-\mathrm{C}(2)-\mathrm{C}(3)$ & $-122.64(11)$ \\
$\mathrm{N}(1)-\mathrm{C}(2)-\mathrm{C}(3)-\mathrm{C}(8)$ & $-159.41(13)$ \\
& \\
&
\end{tabular}


$\mathrm{Si}(1)-\mathrm{C}(2)-\mathrm{C}(3)-\mathrm{C}(8)$

$\mathrm{N}(1)-\mathrm{C}(2)-\mathrm{C}(3)-\mathrm{C}(4)$

$\mathrm{Si}(1)-\mathrm{C}(2)-\mathrm{C}(3)-\mathrm{C}(4)$

$\mathrm{C}(8)-\mathrm{C}(3)-\mathrm{C}(4)-\mathrm{C}(5)$

$\mathrm{C}(2)-\mathrm{C}(3)-\mathrm{C}(4)-\mathrm{C}(5)$

$\mathrm{C}(3)-\mathrm{C}(4)-\mathrm{C}(5)-\mathrm{C}(6)$

$\mathrm{C}(4)-\mathrm{C}(5)-\mathrm{C}(6)-\mathrm{C}(7)$

$\mathrm{C}(5)-\mathrm{C}(6)-\mathrm{C}(7)-\mathrm{C}(8)$

$\mathrm{C}(6)-\mathrm{C}(7)-\mathrm{C}(8)-\mathrm{C}(3)$

$\mathrm{C}(4)-\mathrm{C}(3)-\mathrm{C}(8)-\mathrm{C}(7)$

$\mathrm{C}(2)-\mathrm{C}(3)-\mathrm{C}(8)-\mathrm{C}(7)$

$\mathrm{O}(1)-\mathrm{Si}(1)-\mathrm{C}(9)-\mathrm{C}(11)$

$\mathrm{C}(13)-\mathrm{Si}(1)-\mathrm{C}(9)-\mathrm{C}(11)$

$\mathrm{C}(2)-\mathrm{Si}(1)-\mathrm{C}(9)-\mathrm{C}(11)$

$\mathrm{O}(1)-\mathrm{Si}(1)-\mathrm{C}(9)-\mathrm{C}(10)$

C(13)-Si(1)-C(9)-C(10)

$\mathrm{C}(2)-\mathrm{Si}(1)-\mathrm{C}(9)-\mathrm{C}(10)$

$\mathrm{O}(1)-\mathrm{Si}(1)-\mathrm{C}(9)-\mathrm{C}(12)$

C(13)-Si(1)-C(9)-C(12)

$\mathrm{C}(2)-\mathrm{Si}(1)-\mathrm{C}(9)-\mathrm{C}(12)$

$\mathrm{O}(1)-\mathrm{Si}(1)-\mathrm{C}(13)-\mathrm{C}(14)$

C(9)-Si(1)-C(13)-C(14)

C(2)-Si(1)-C(13)-C(14)

$\mathrm{O}(1)-\mathrm{Si}(1)-\mathrm{C}(13)-\mathrm{C}(16)$

C(9)-Si(1)-C(13)-C(16)

C(2)-Si(1)-C(13)-C(16)

$\mathrm{O}(1)-\mathrm{Si}(1)-\mathrm{C}(13)-\mathrm{C}(15)$

C(9)-Si(1)-C(13)-C(15)

C(2)-Si(1)-C(13)-C(15)

C(24)-N(2)-C(17)-N(1)

$\mathrm{C}(24)-\mathrm{N}(2)-\mathrm{C}(17)-\mathrm{C}(18)$

$\mathrm{C}(1)-\mathrm{N}(1)-\mathrm{C}(17)-\mathrm{N}(2)$

$\mathrm{C}(2)-\mathrm{N}(1)-\mathrm{C}(17)-\mathrm{N}(2)$

C(1)-N(1)-C(17)-C(18)

$\mathrm{C}(2)-\mathrm{N}(1)-\mathrm{C}(17)-\mathrm{C}(18)$

$\mathrm{N}(2)-\mathrm{C}(17)-\mathrm{C}(18)-\mathrm{C}(23)$

$\mathrm{N}(1)-\mathrm{C}(17)-\mathrm{C}(18)-\mathrm{C}(23)$

$\mathrm{N}(2)-\mathrm{C}(17)-\mathrm{C}(18)-\mathrm{C}(19)$
83.19(15)

19.21(19)

$-98.19(15)$

$0.4(2)$

$-178.18(14)$

$-1.0(2)$

$0.0(2)$

$1.5(3)$

$-2.1(3)$

1.1(2)

179.78(14)

$-163.29(12)$

77.31(14)

$-62.10(14)$

74.77(12)

$-44.63(14)$

175.95(11)

$-42.66(13)$

$-162.06(12)$

$58.53(14)$

-166.97(12)

$-47.22(14)$

93.81(13)

72.51(12)

$-167.75(11)$

$-26.72(13)$

$-44.83(13)$

74.92(14)

$-144.05(12)$

122.63(14)

$-109.91(16)$

51.68(17)

$-145.80(12)$

$-76.46(17)$

86.06(15)

125.99(15)

$-107.30(15)$

$-54.12(19)$ 


$\begin{array}{lc}\mathrm{N}(1)-\mathrm{C}(17)-\mathrm{C}(18)-\mathrm{C}(19) & 72.59(18) \\ \mathrm{C}(23)-\mathrm{C}(18)-\mathrm{C}(19)-\mathrm{C}(20) & 0.1(2) \\ \mathrm{C}(17)-\mathrm{C}(18)-\mathrm{C}(19)-\mathrm{C}(20) & -179.83(15) \\ \mathrm{C}(18)-\mathrm{C}(19)-\mathrm{C}(20)-\mathrm{C}(21) & -0.3(3) \\ \mathrm{C}(19)-\mathrm{C}(20)-\mathrm{C}(21)-\mathrm{C}(22) & 0.4(3) \\ \mathrm{C}(20)-\mathrm{C}(21)-\mathrm{C}(22)-\mathrm{C}(23) & -0.4(3) \\ \mathrm{C}(19)-\mathrm{C}(18)-\mathrm{C}(23)-\mathrm{C}(22) & 0.0(2) \\ \mathrm{C}(17)-\mathrm{C}(18)-\mathrm{C}(23)-\mathrm{C}(22) & 179.85(14) \\ \mathrm{C}(21)-\mathrm{C}(22)-\mathrm{C}(23)-\mathrm{C}(18) & 0.2(3) \\ \mathrm{C}(25)-\mathrm{O}(3)-\mathrm{C}(24)-\mathrm{O}(4) & -7.3(2) \\ \mathrm{C}(25)-\mathrm{O}(3)-\mathrm{C}(24)-\mathrm{N}(2) & 173.20(13) \\ \mathrm{C}(17)-\mathrm{N}(2)-\mathrm{C}(24)-\mathrm{O}(4) & -1.3(2) \\ \mathrm{C}(17)-\mathrm{N}(2)-\mathrm{C}(24)-\mathrm{O}(3) & 178.22(13) \\ \mathrm{C}(24)-\mathrm{O}(3)-\mathrm{C}(25)-\mathrm{C}(27) & 60.72(19) \\ \mathrm{C}(24)-\mathrm{O}(3)-\mathrm{C}(25)-\mathrm{C}(26) & 179.77(16) \\ \mathrm{C}(24)-\mathrm{O}(3)-\mathrm{C}(25)-\mathrm{C}(28) & -63.45(19)\end{array}$




\section{Selected Spectra}

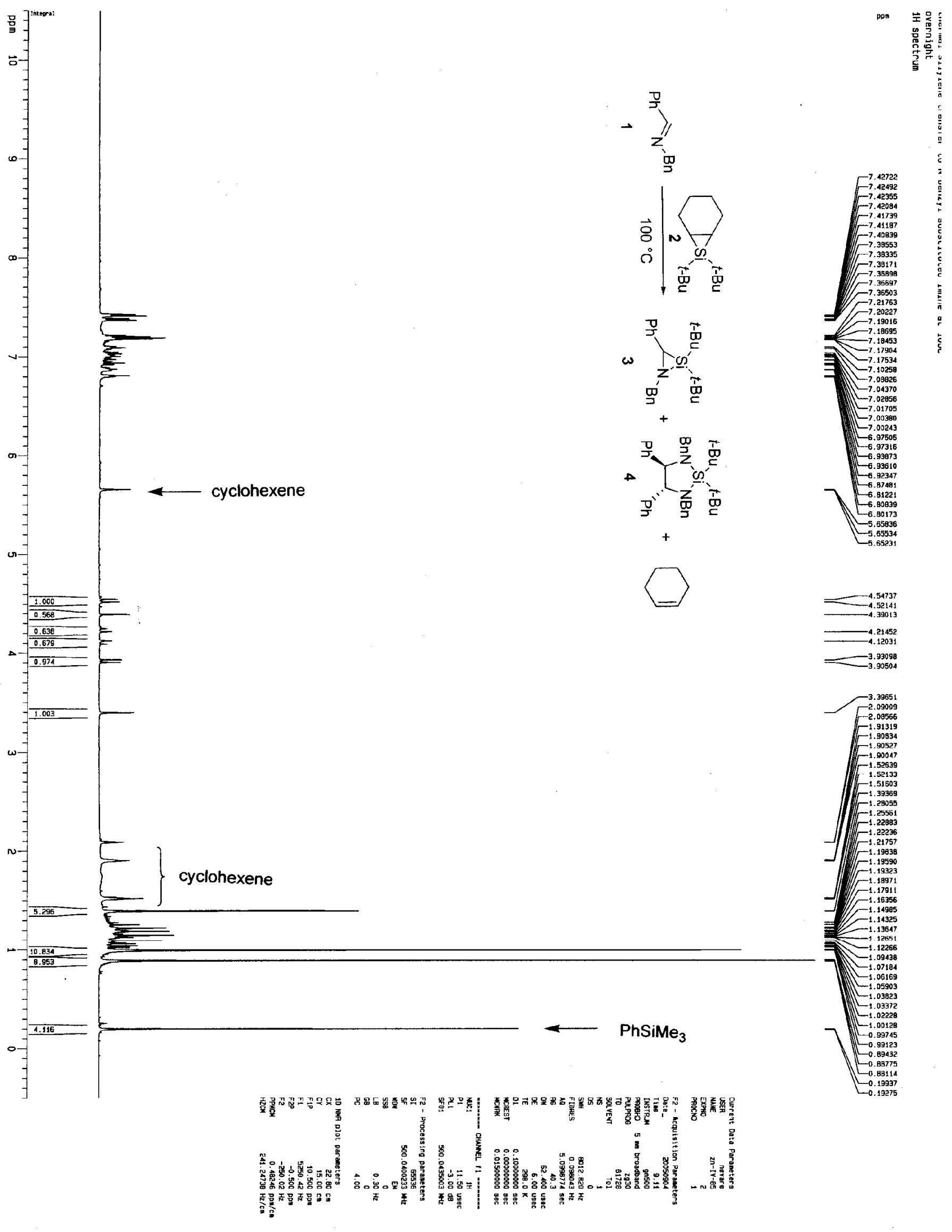




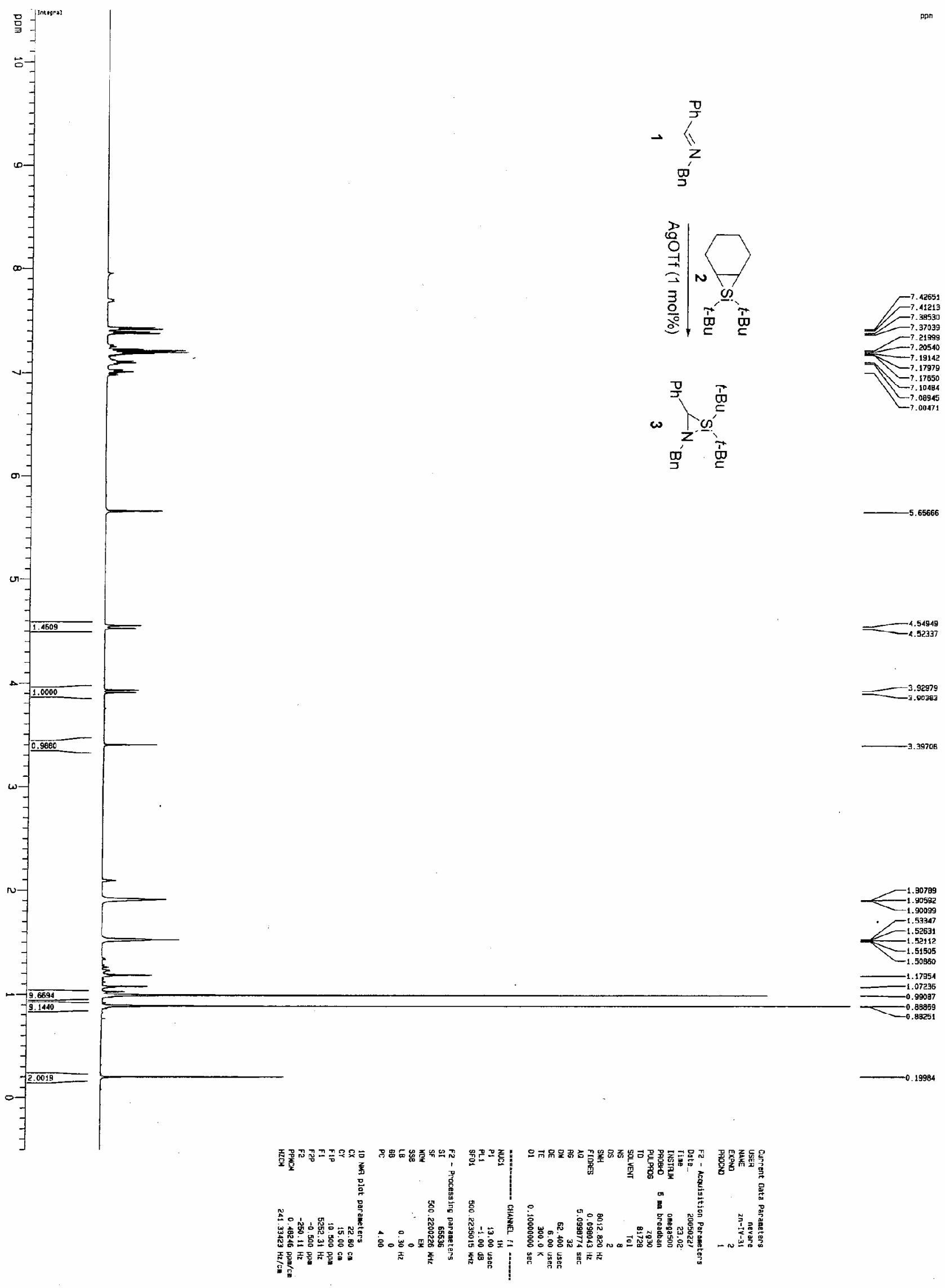




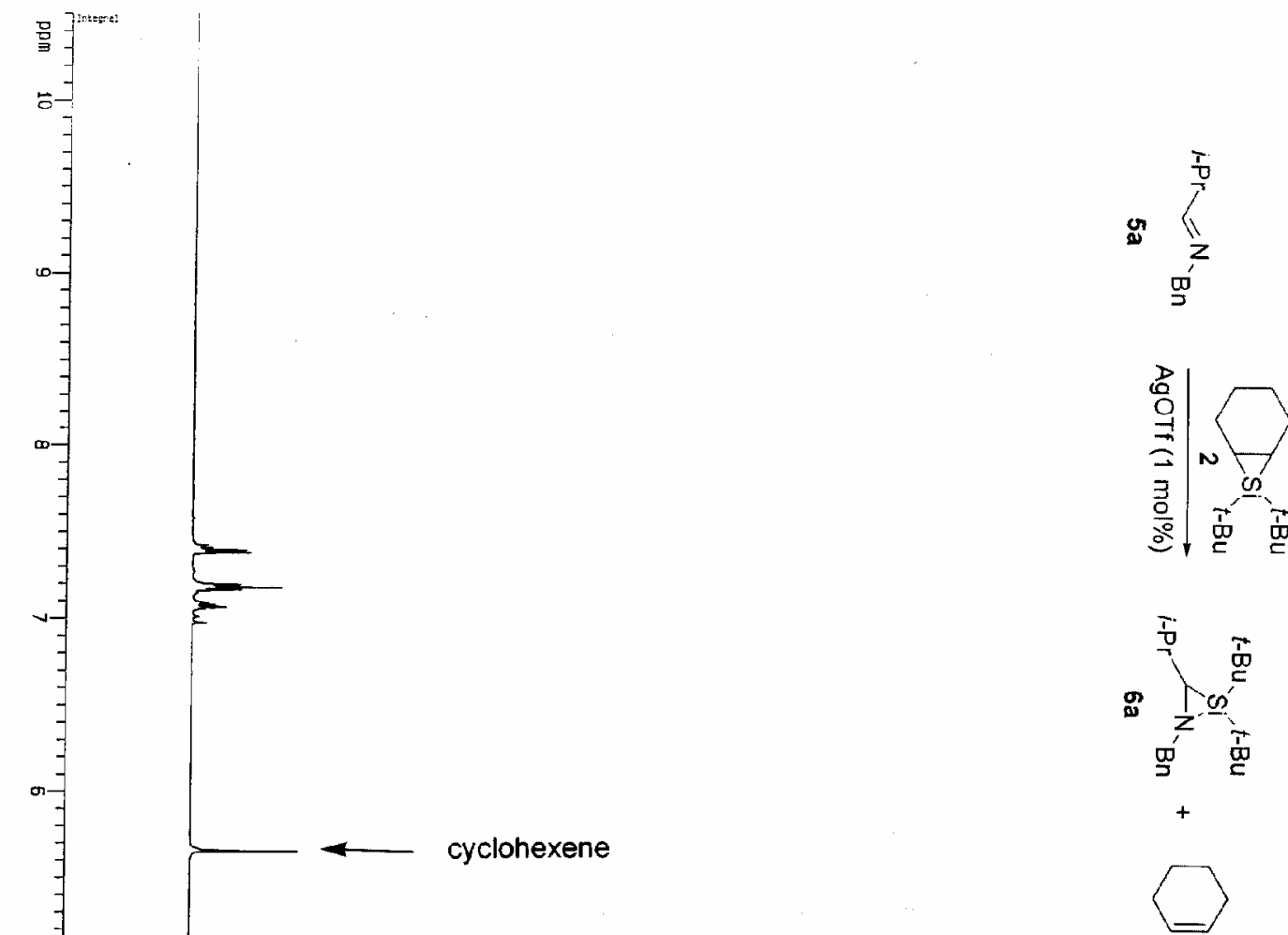

-
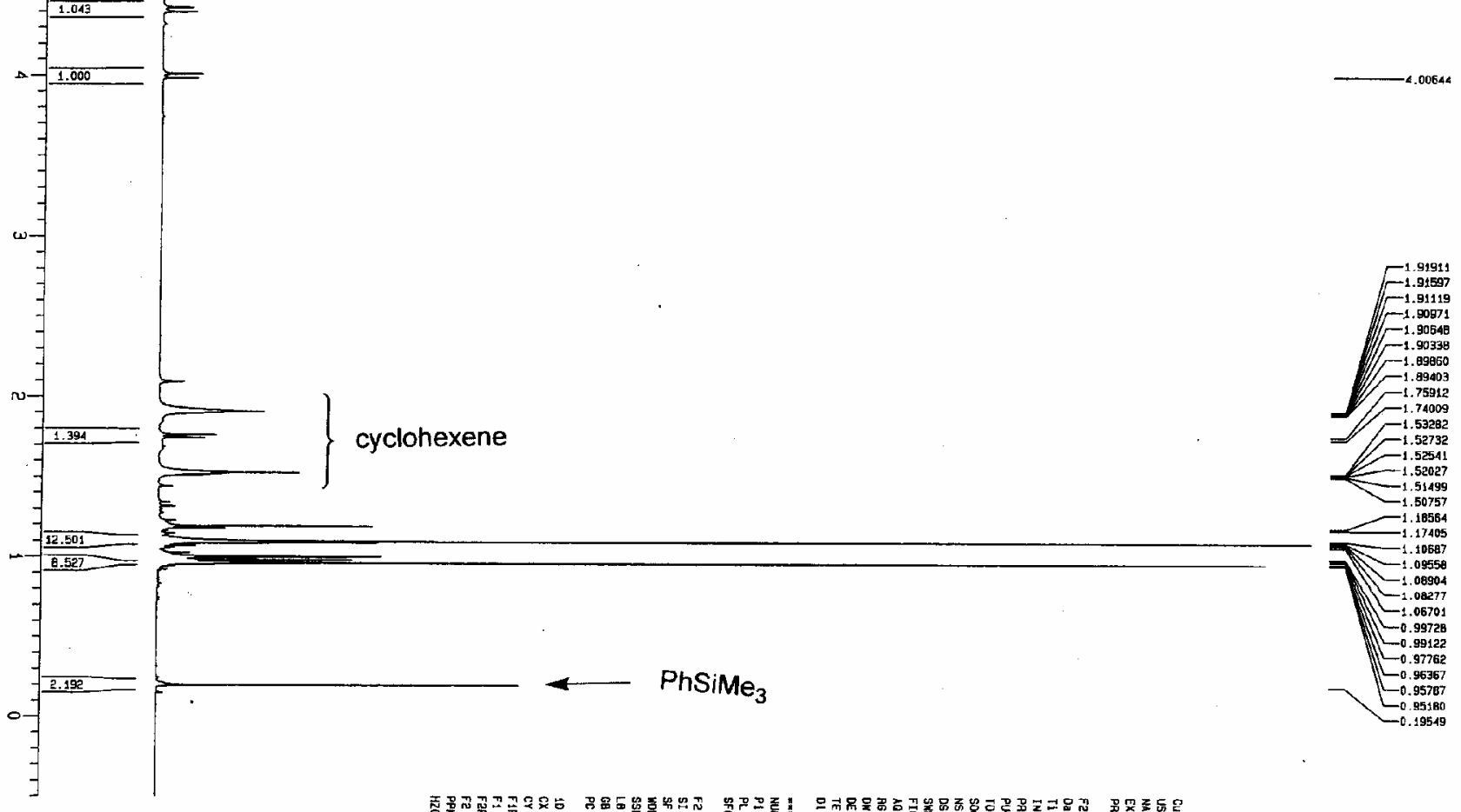


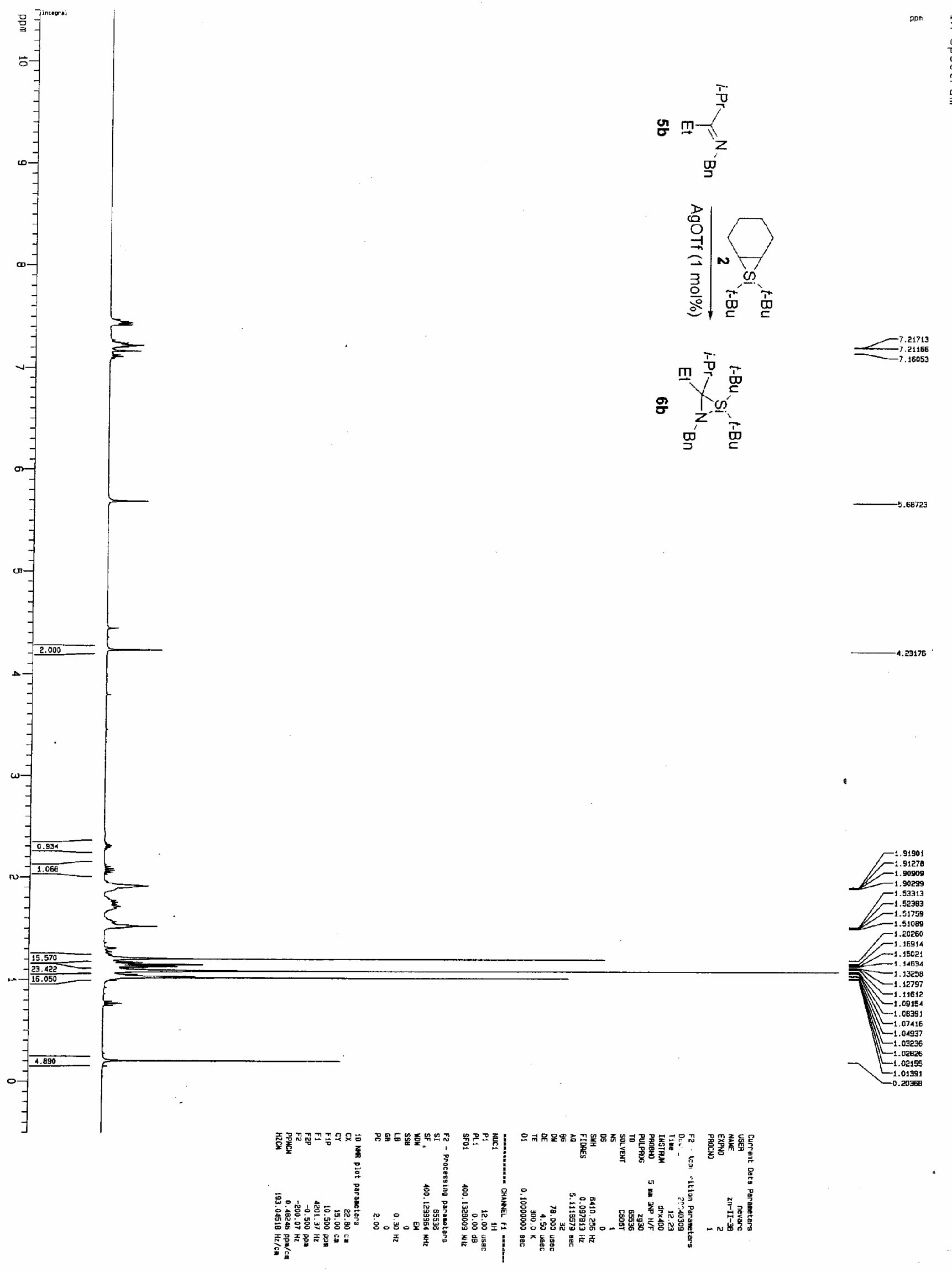




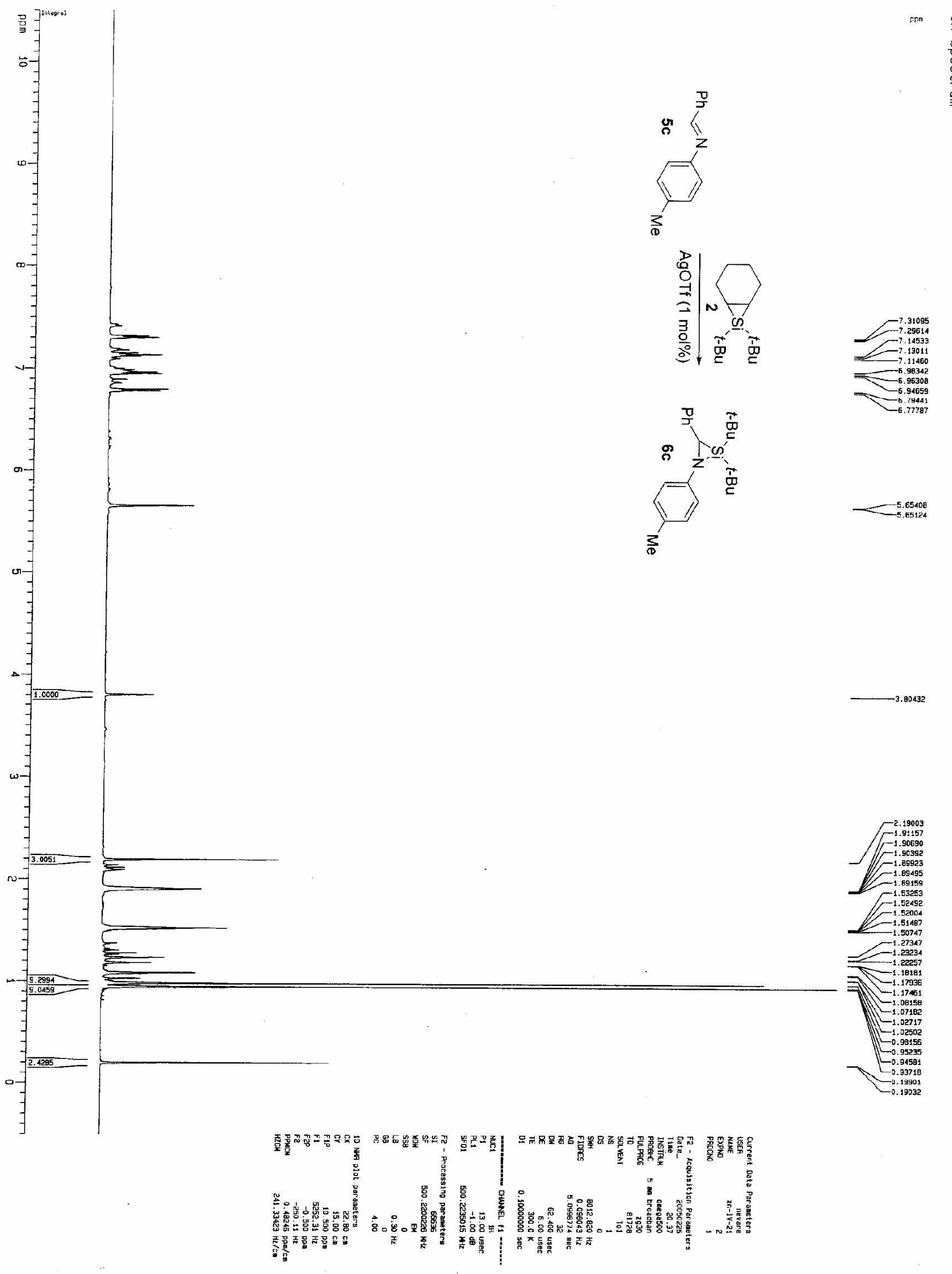




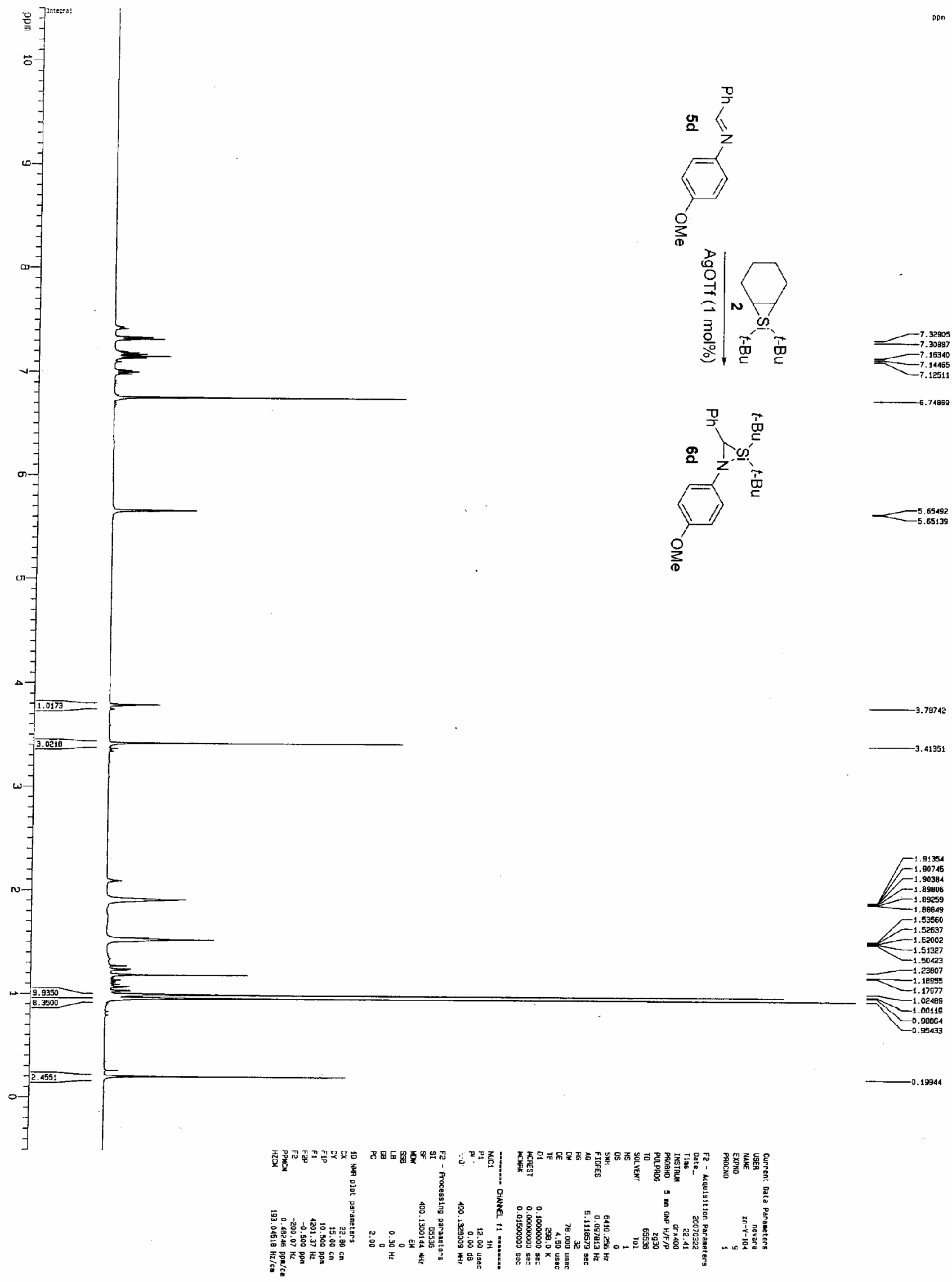



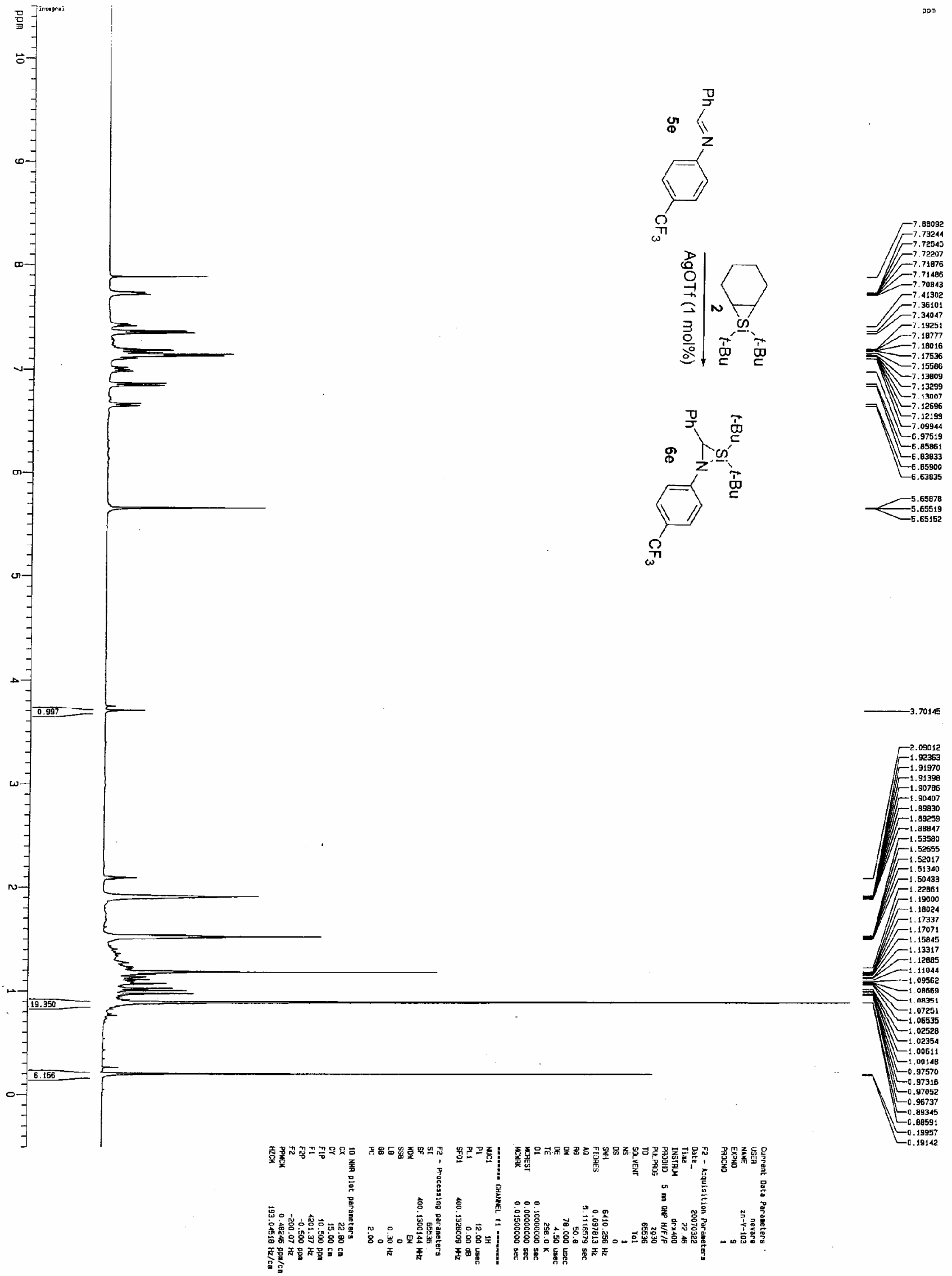


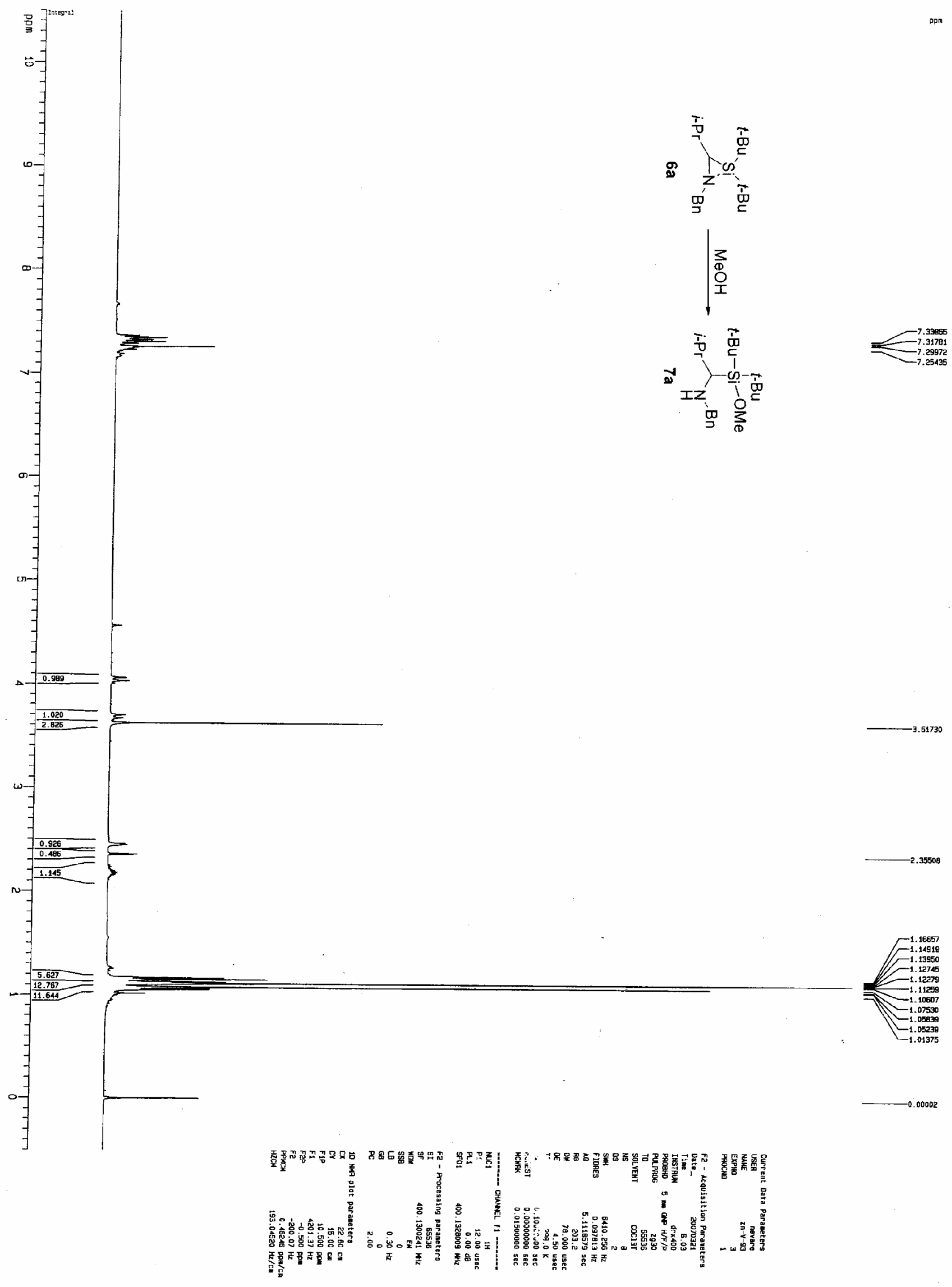



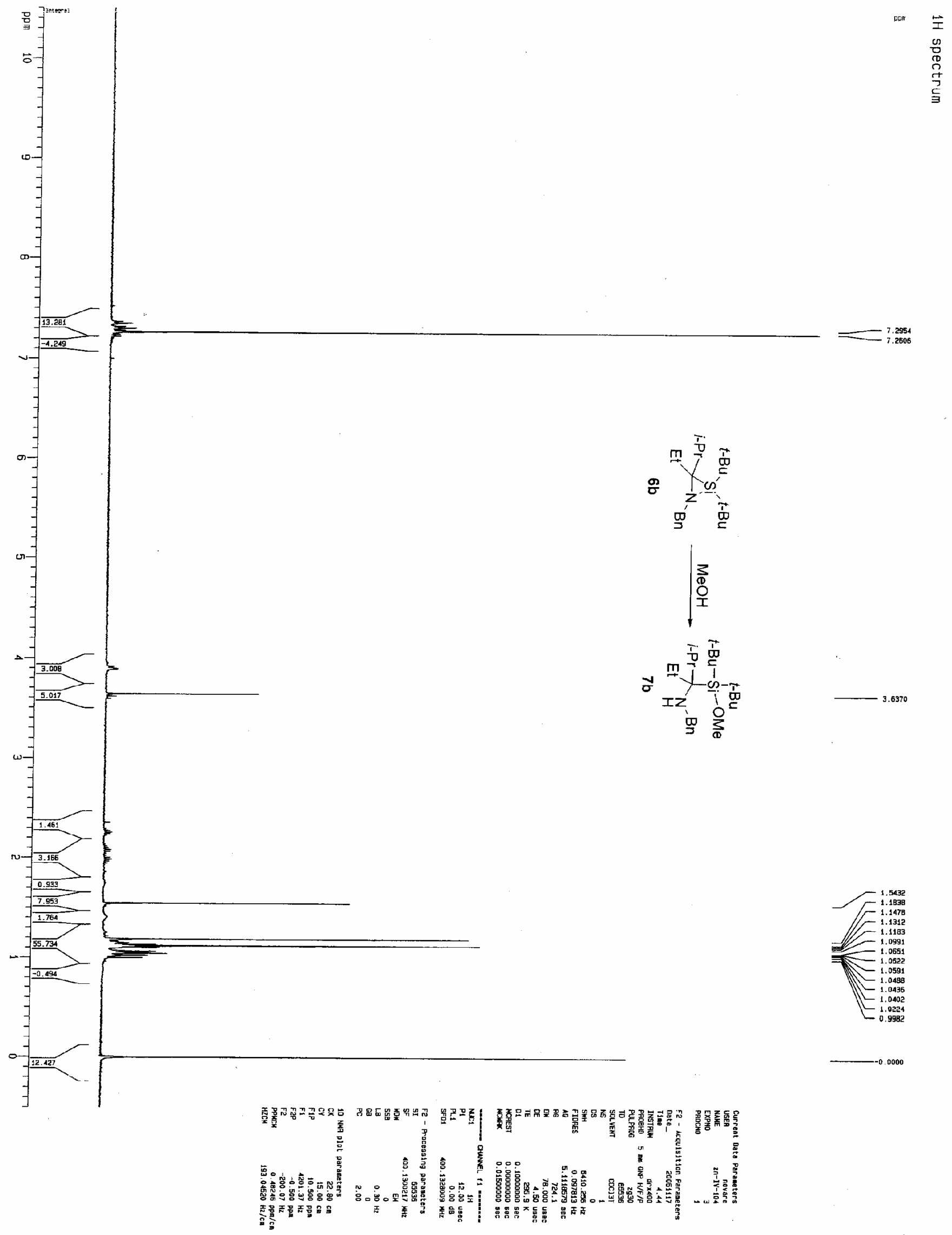


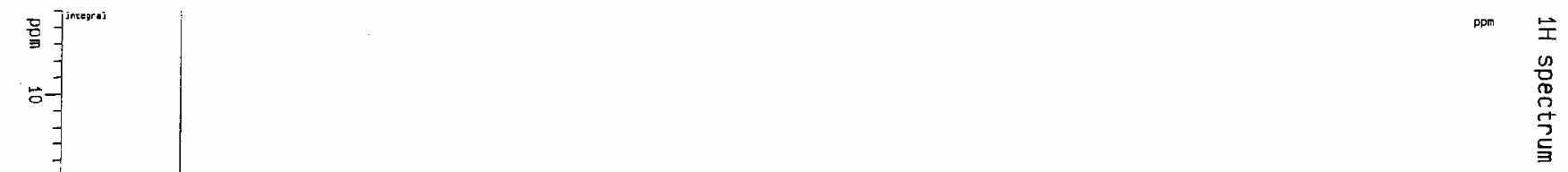

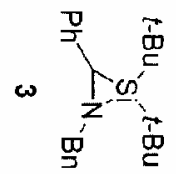
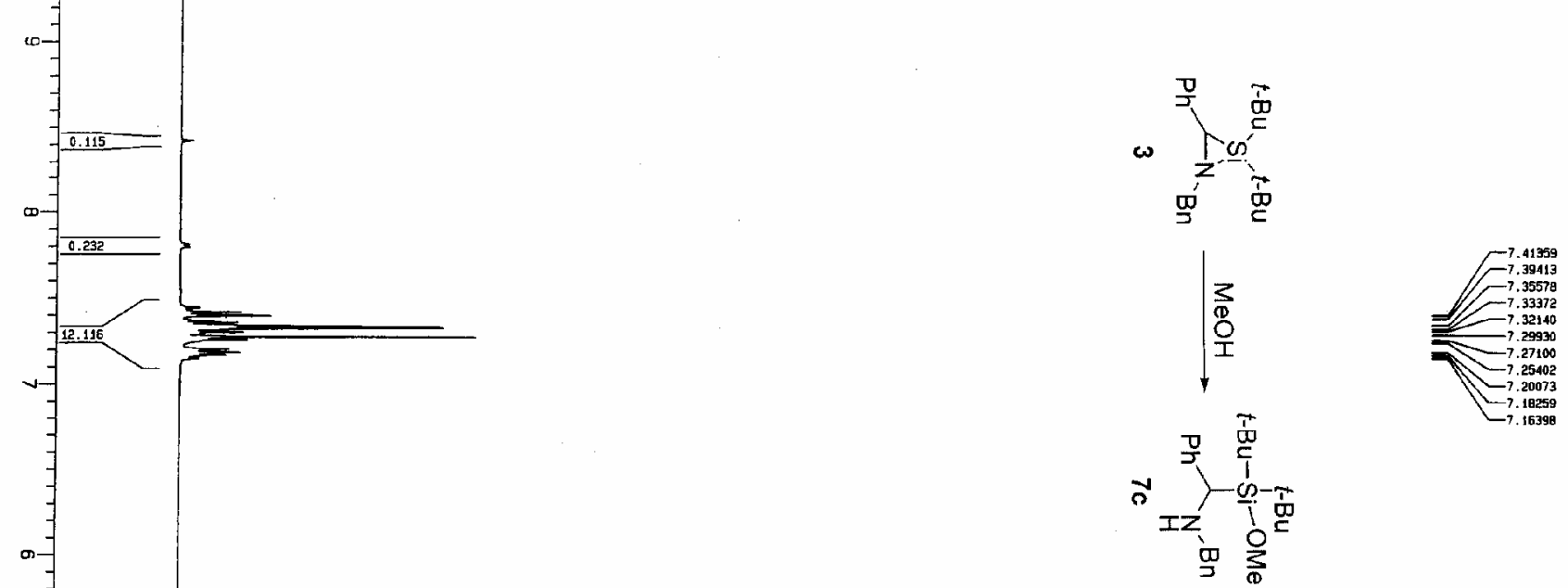

-
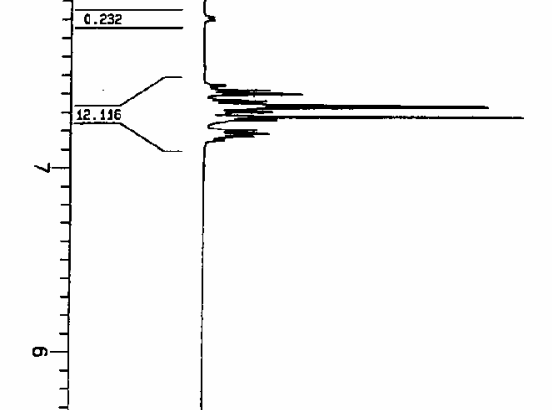

c.
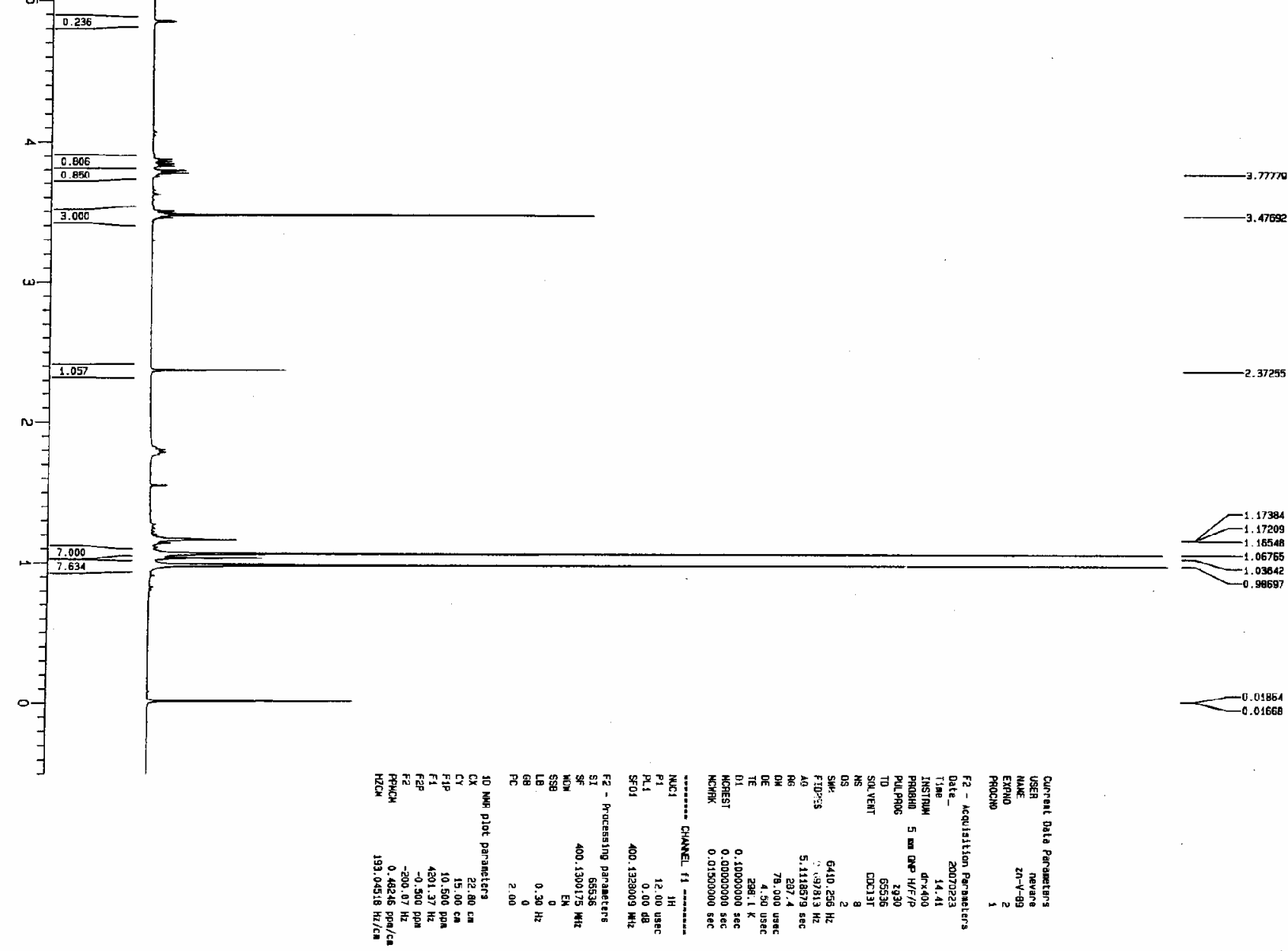


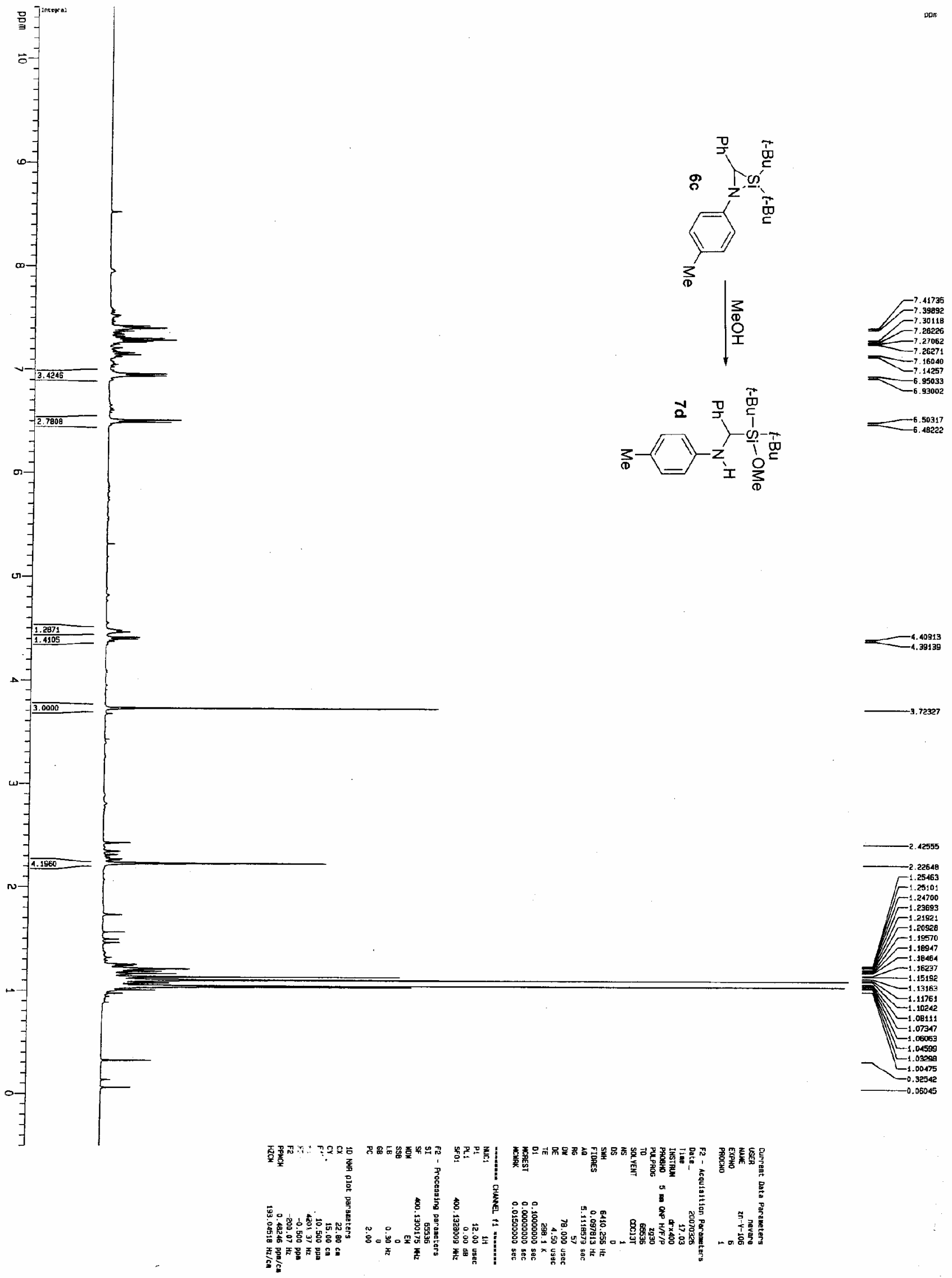




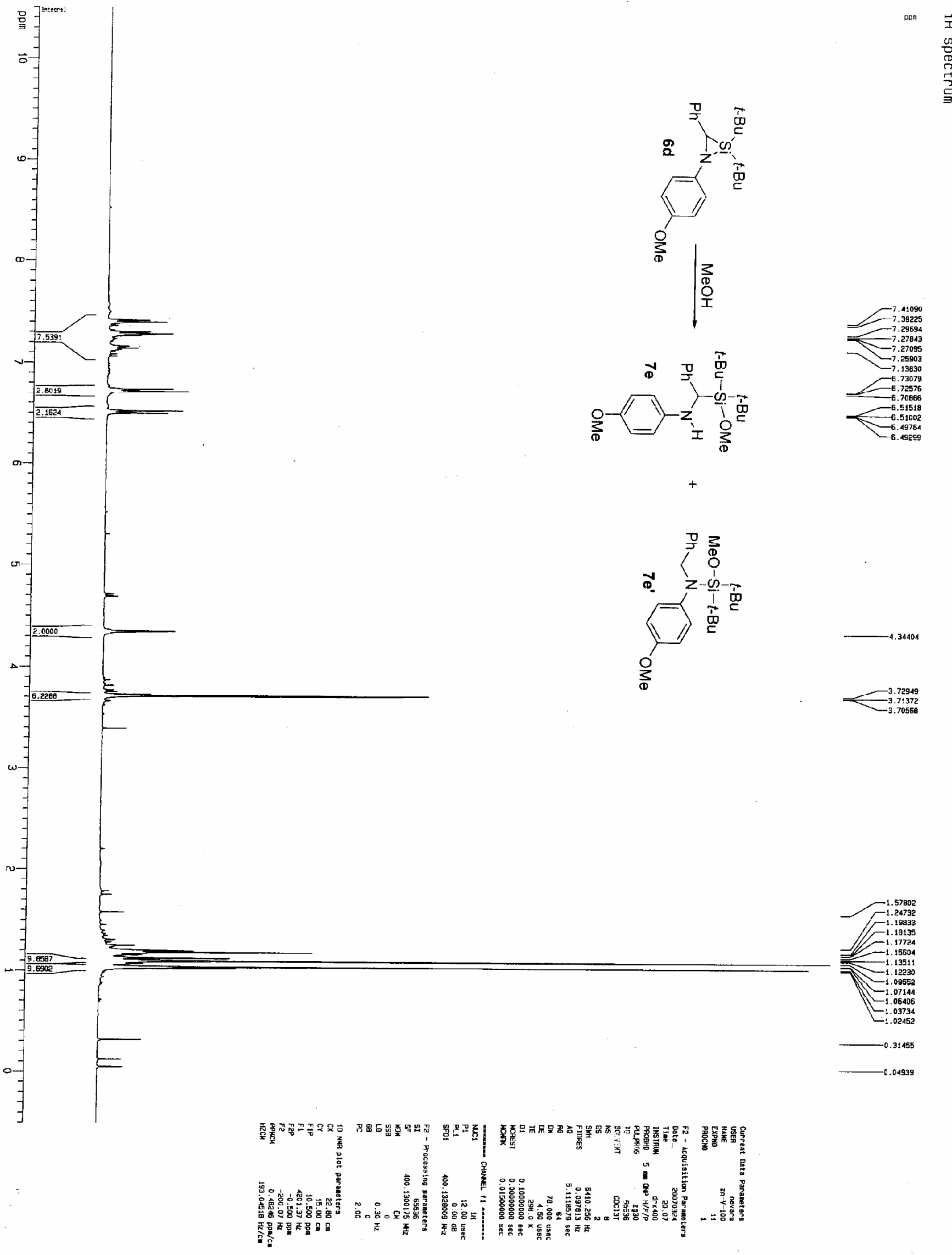



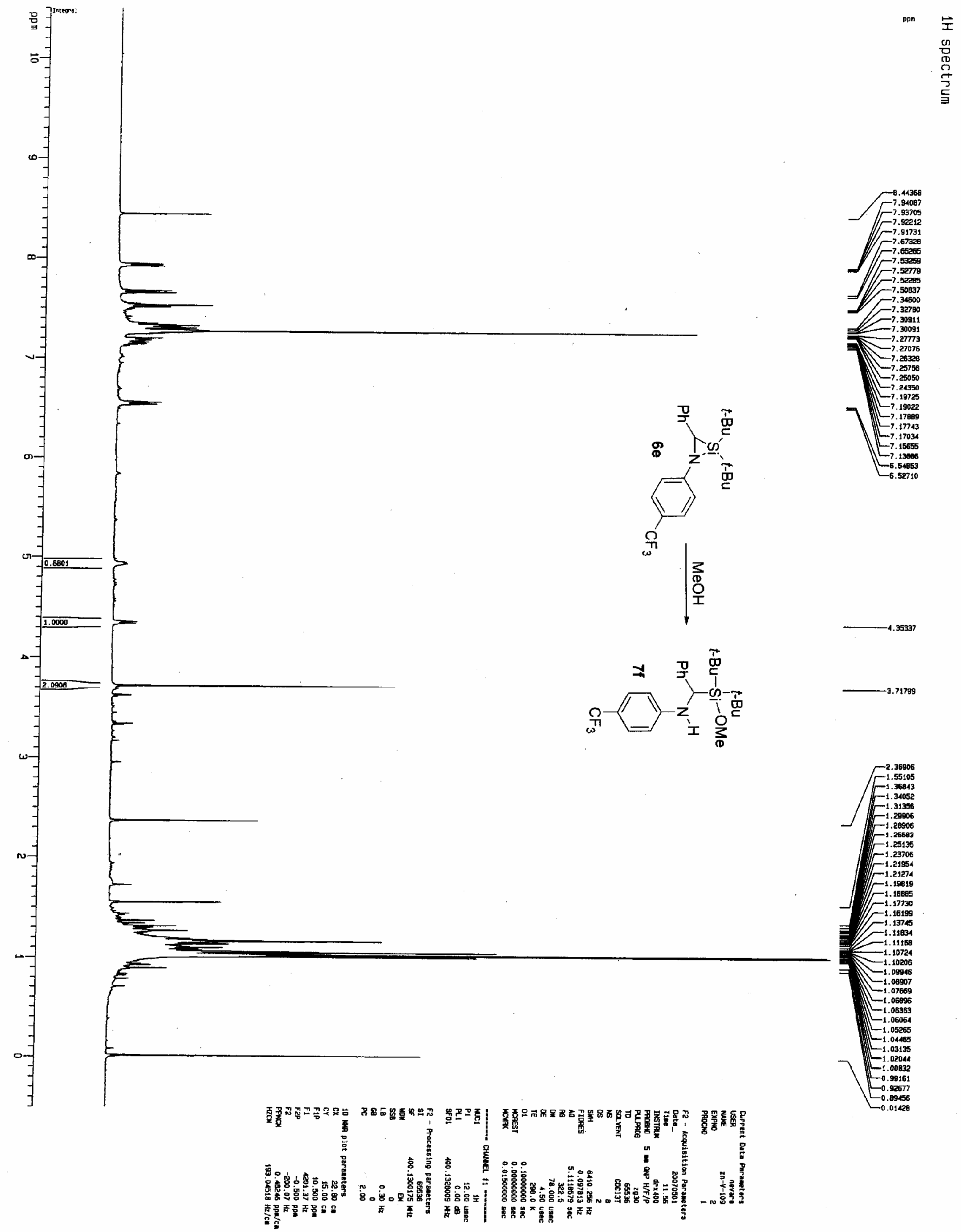


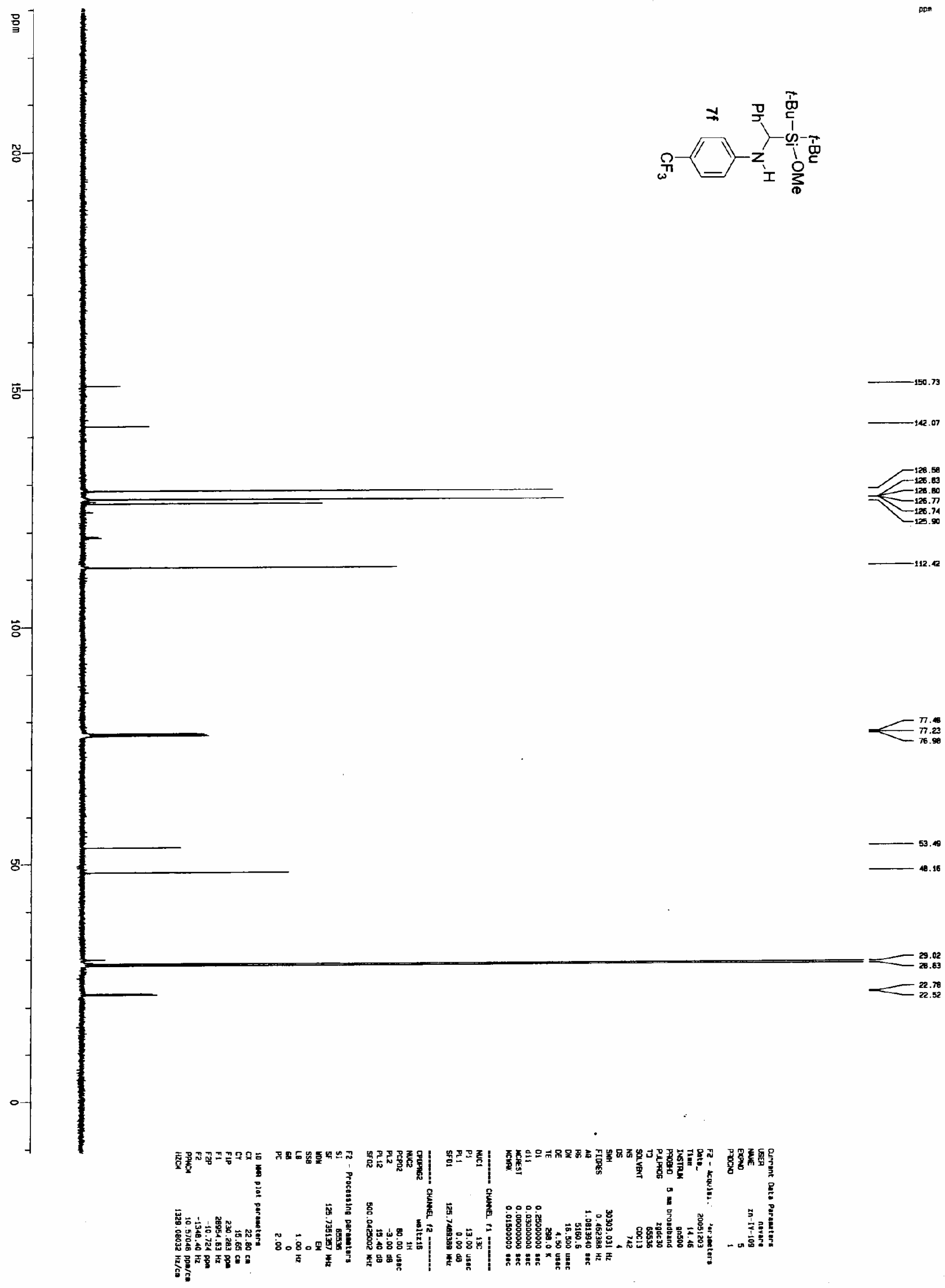




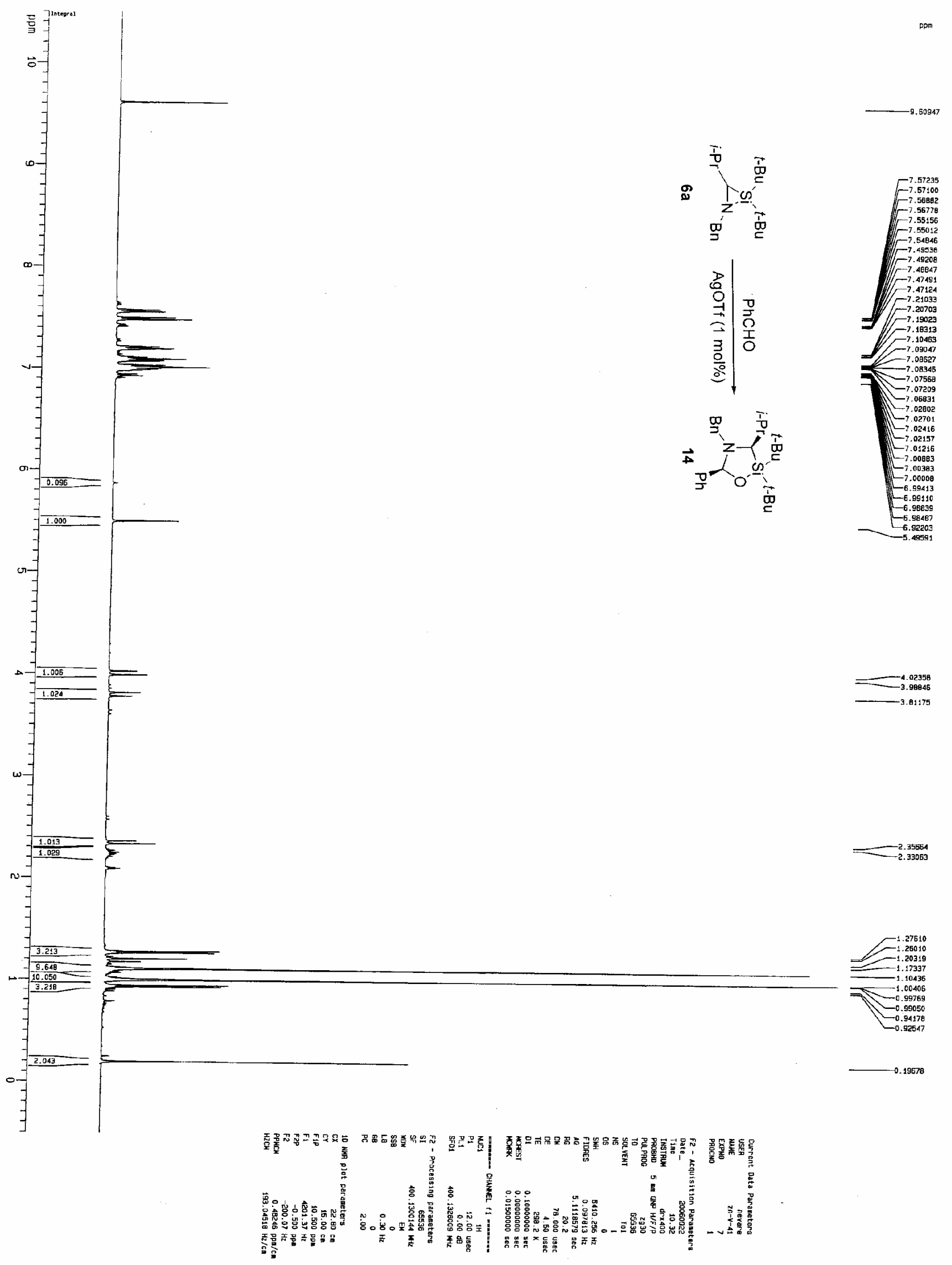



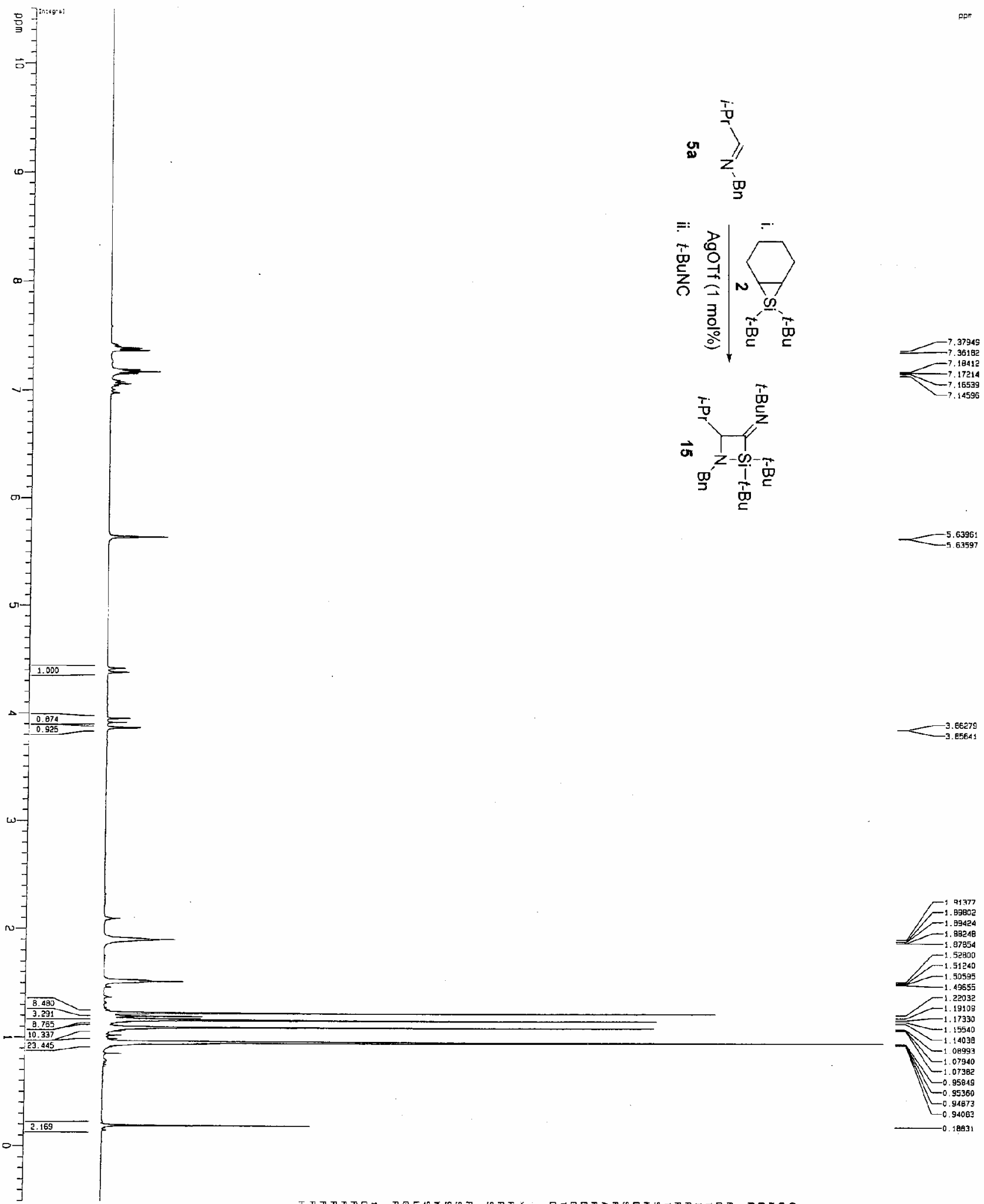

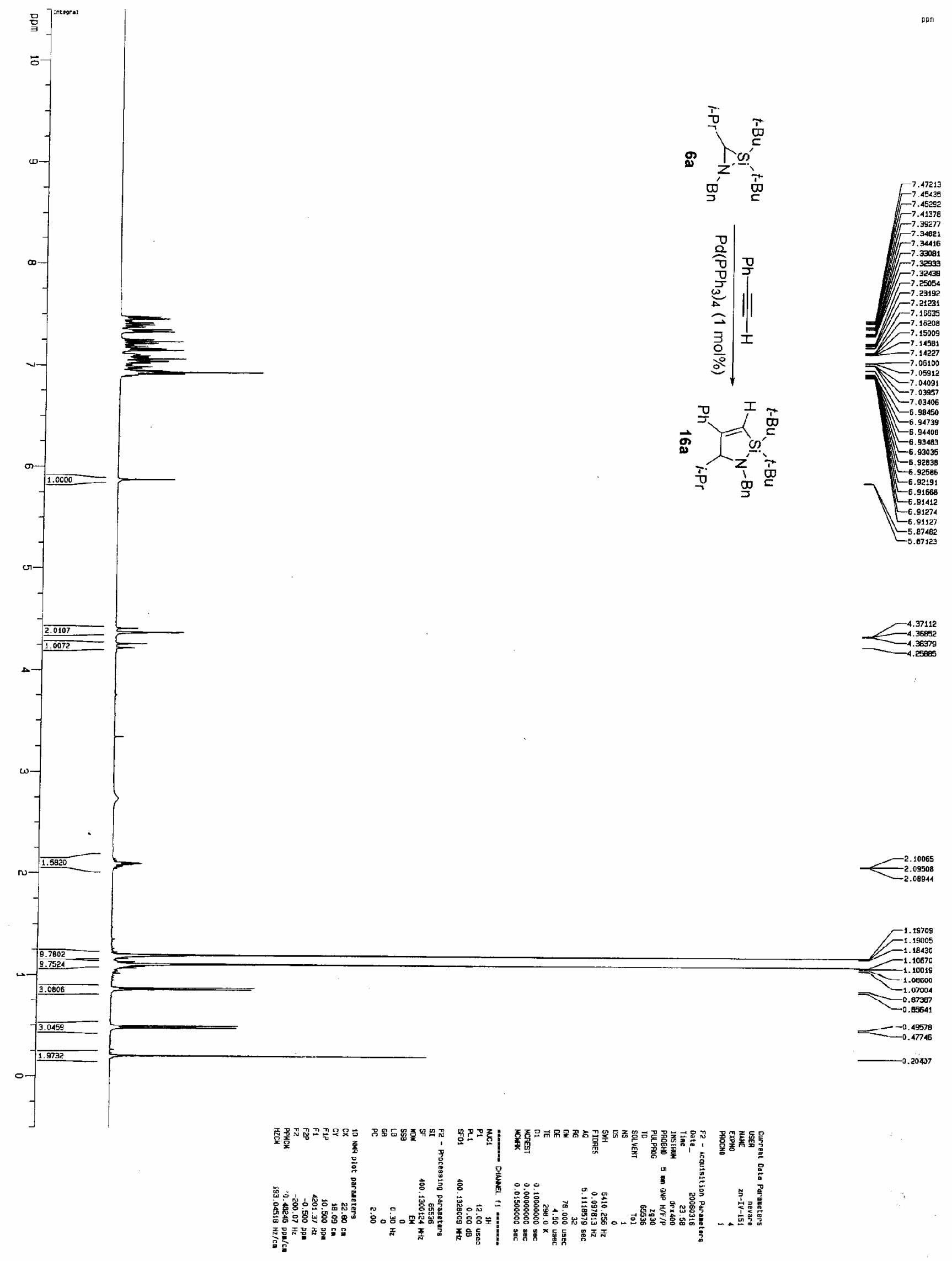


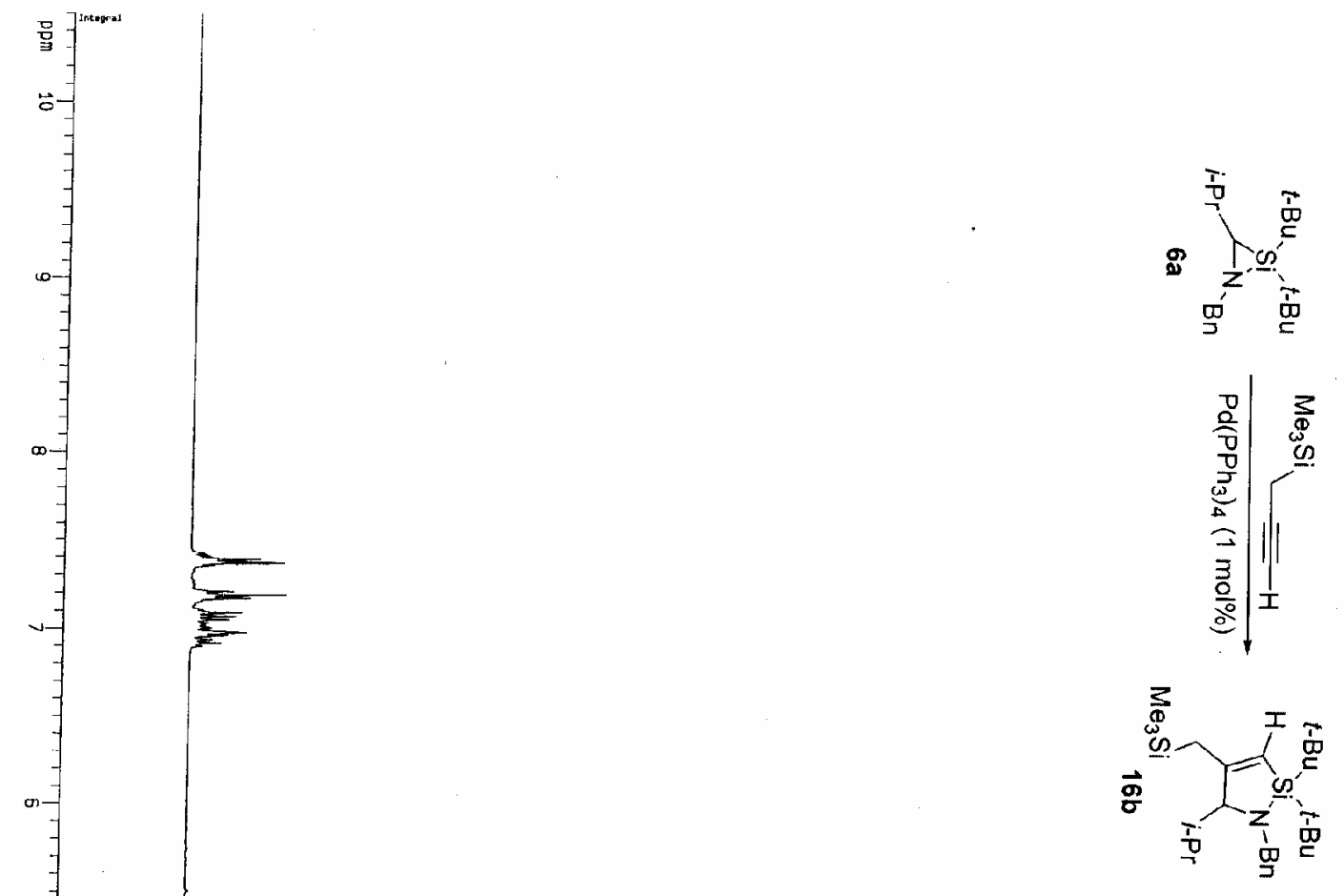

ppat

足
号
号
吉
至

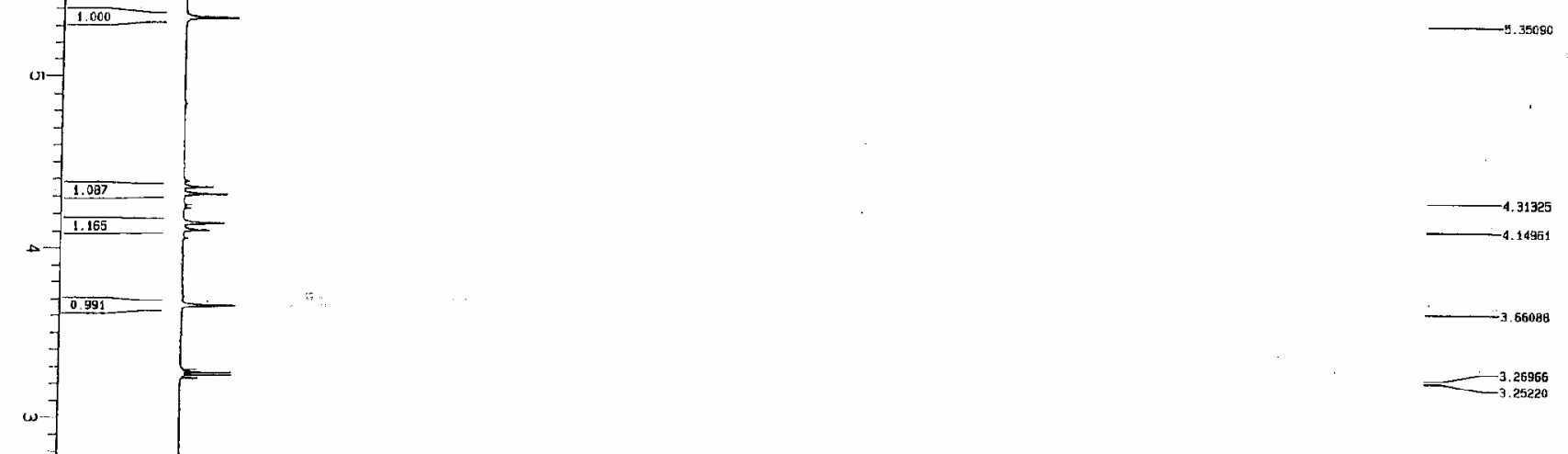

n

$=$

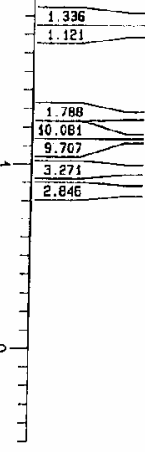

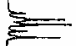
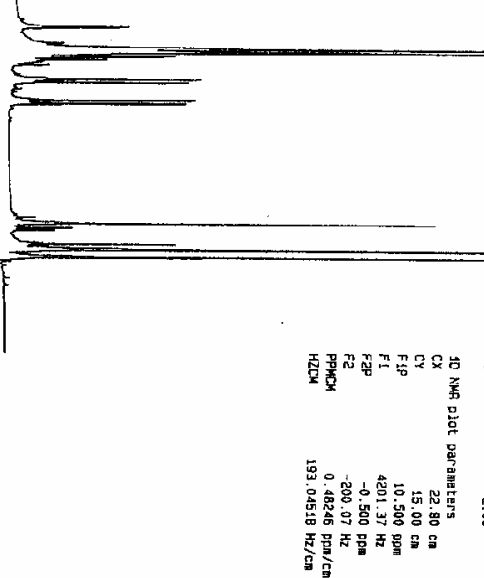

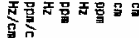

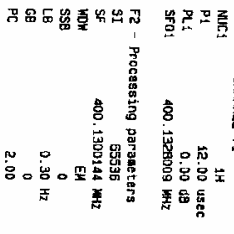

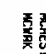

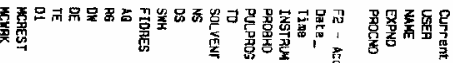

-2. 11999

$-1.76203$

$F_{1.5654}^{1.5334}$

$F^{1.253941}$

$p^{-1.123999}$

${ }_{-1.09844}^{1.10234}$

$a-0.9655$

$-0.46231$

$\overbrace{-0.175828}^{-0.20279}$

$=0.017287$

$-0.06150$

$C_{0.04981}^{-0.04563}$

$\subset^{-0.04981}$ 

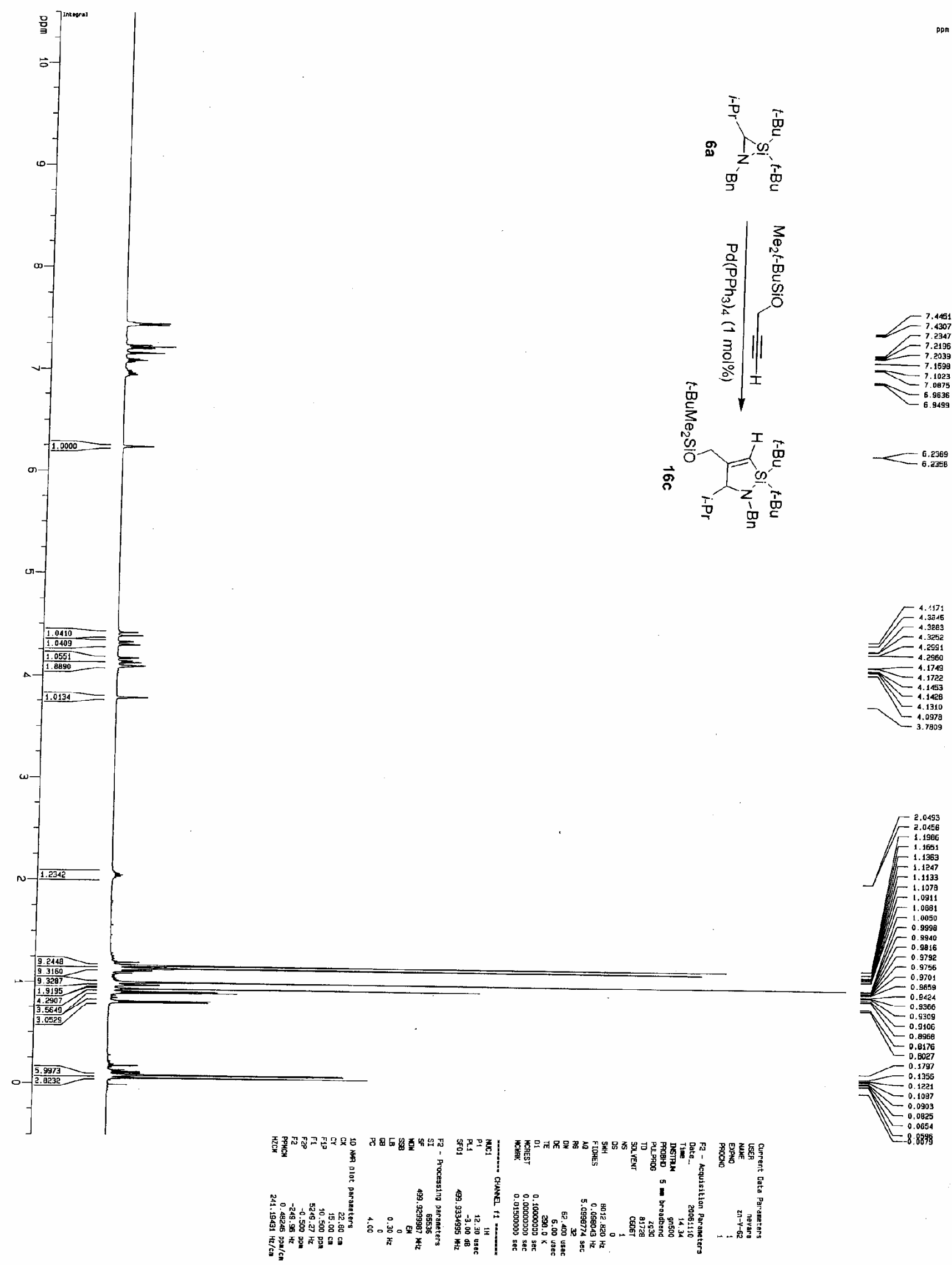


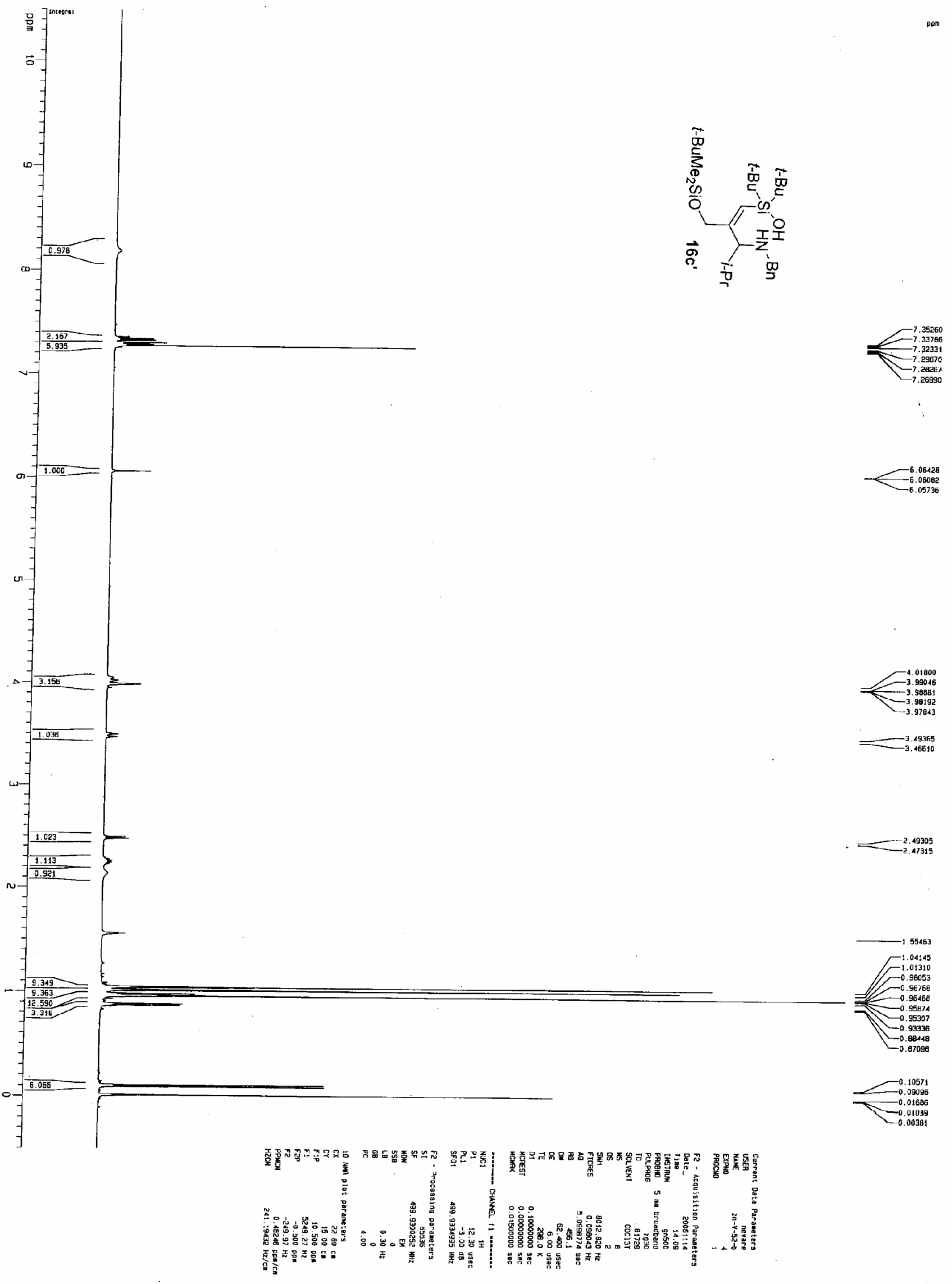




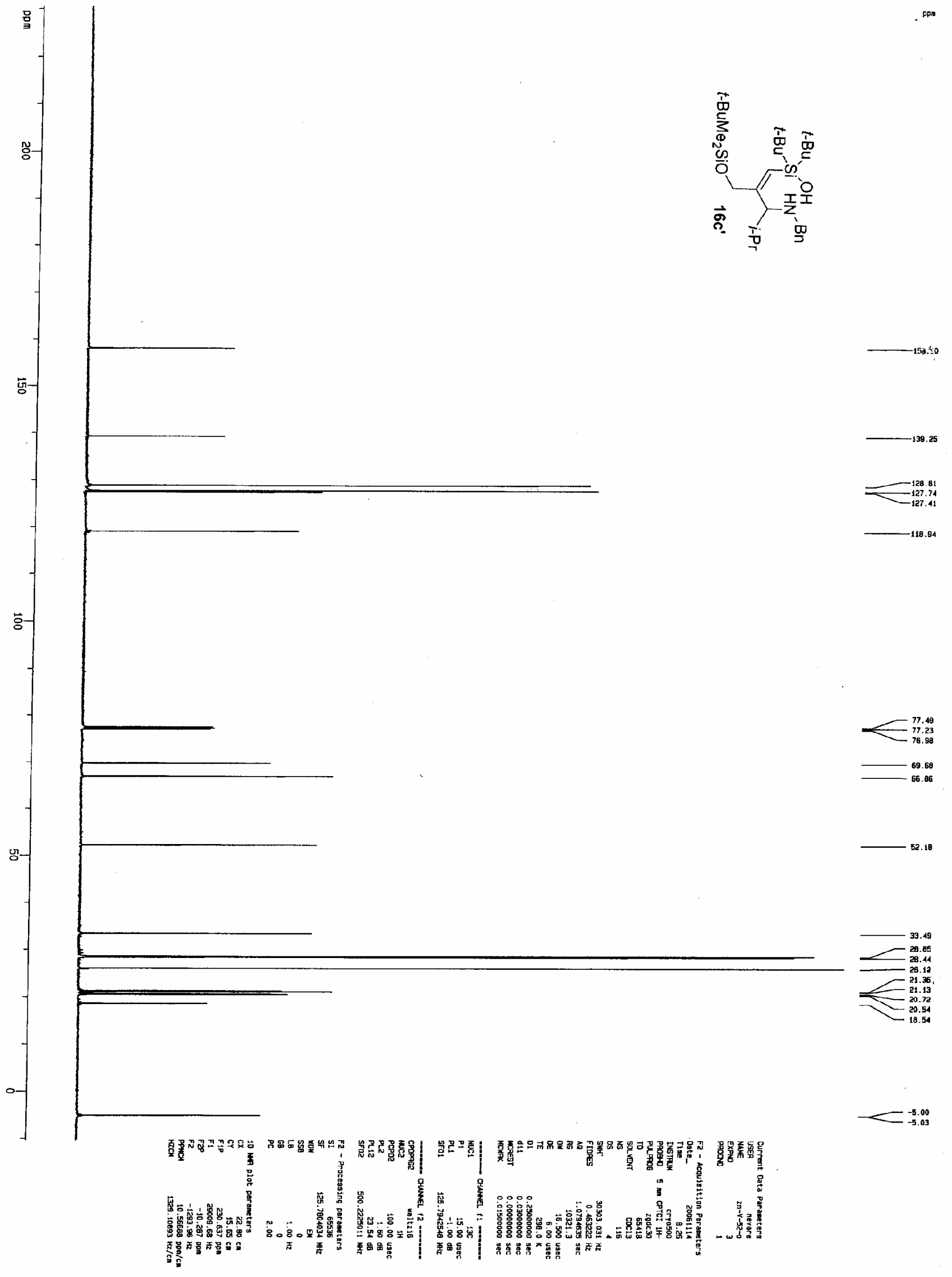




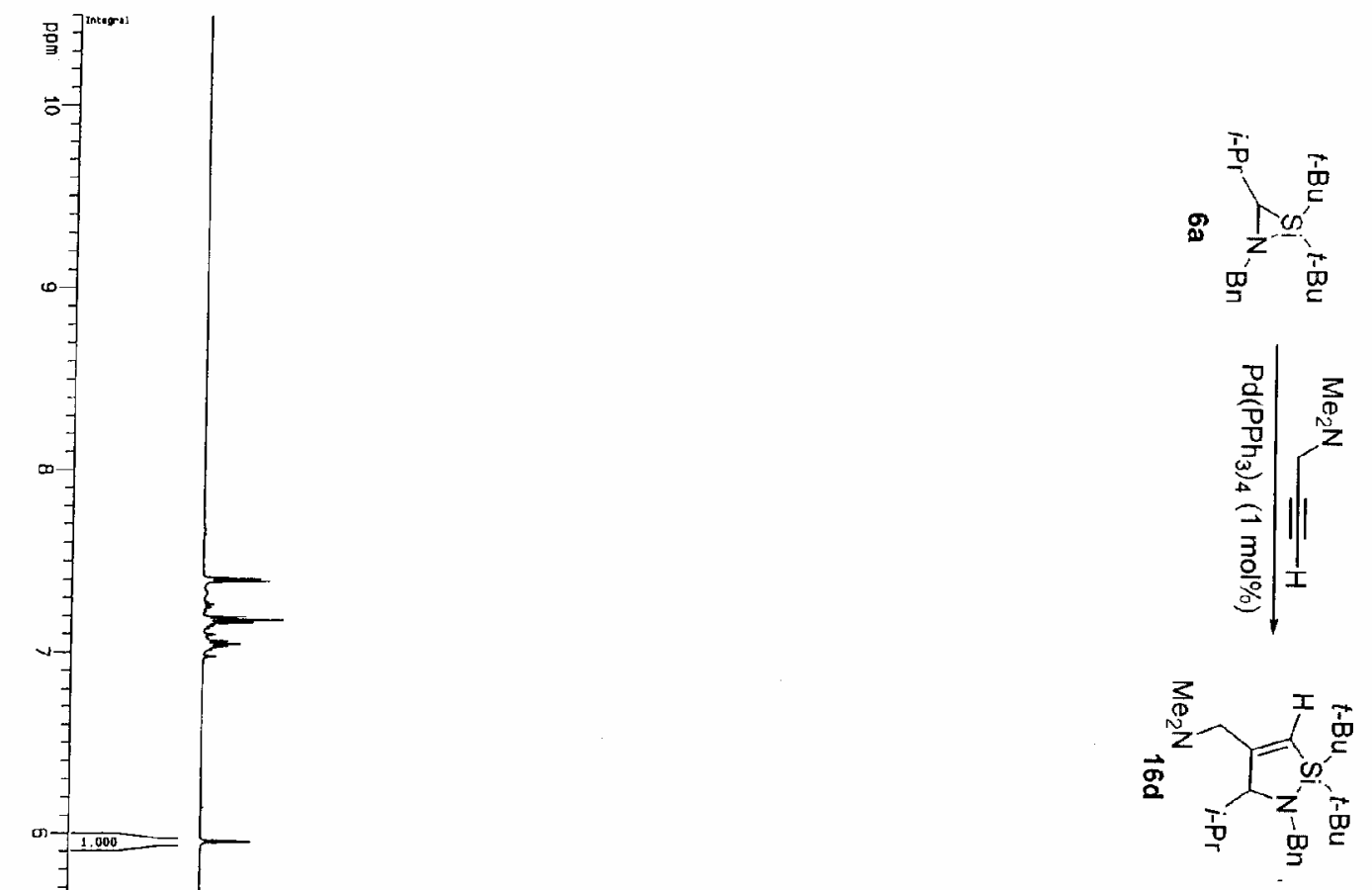

pom 宇

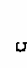
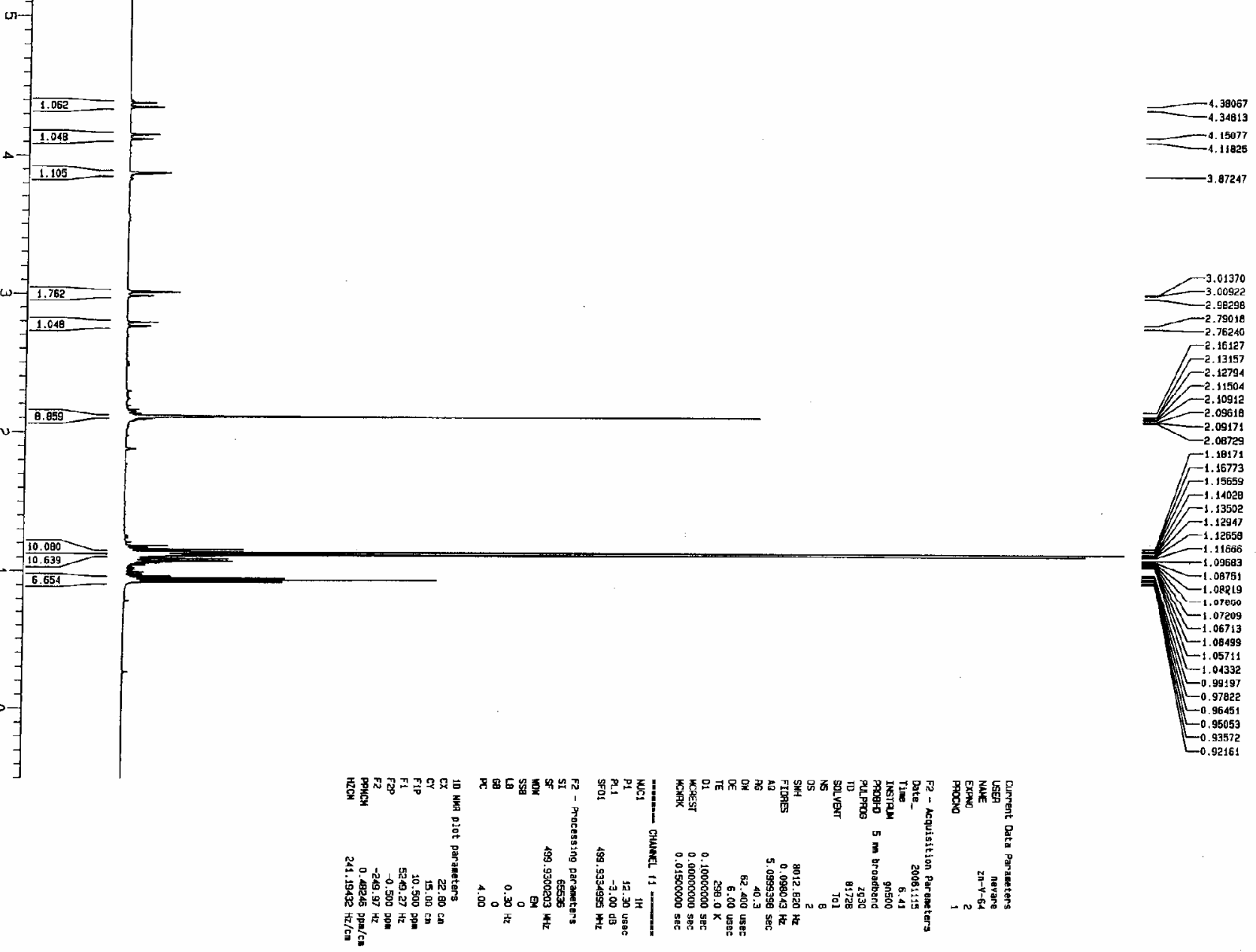

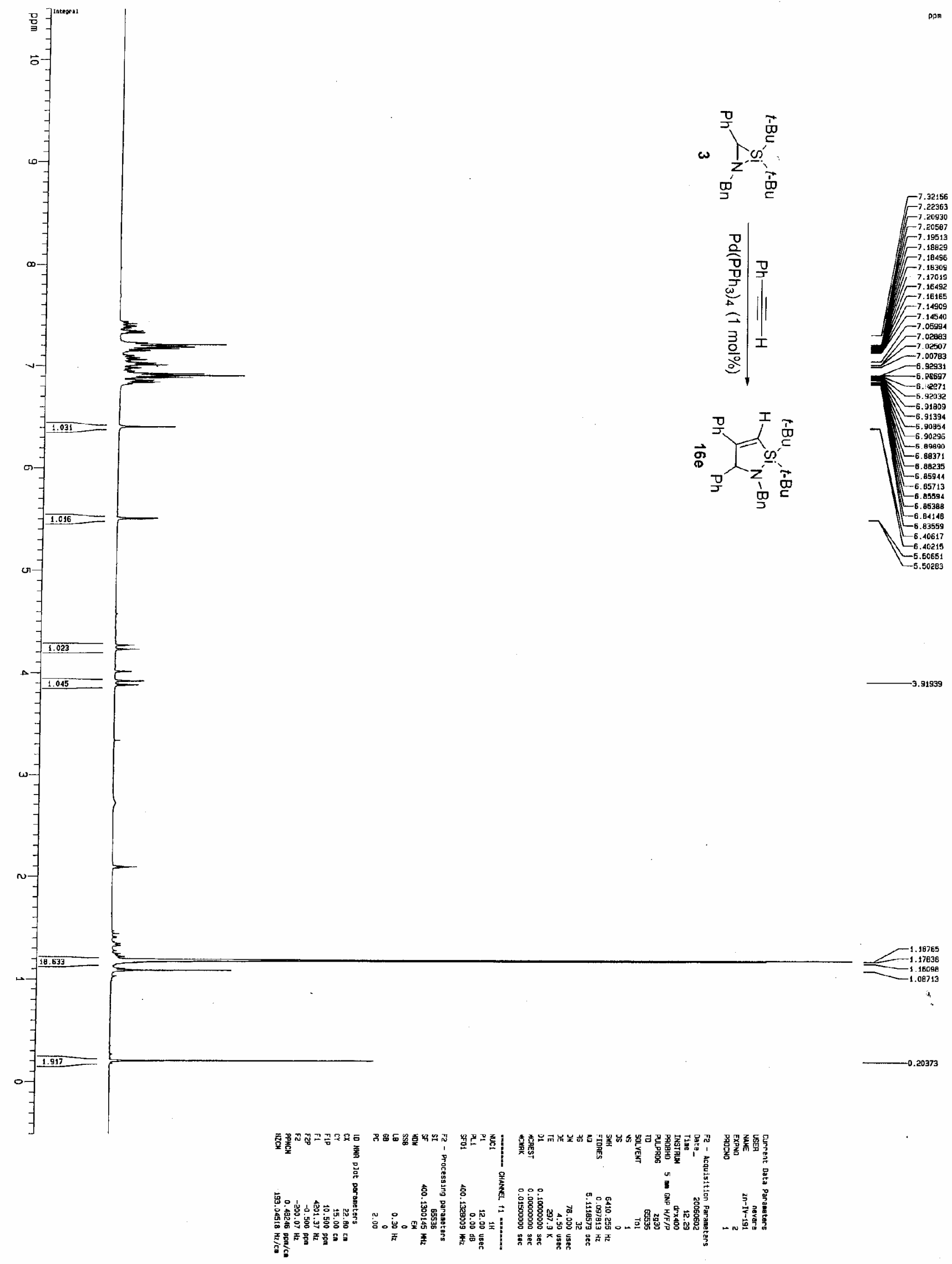


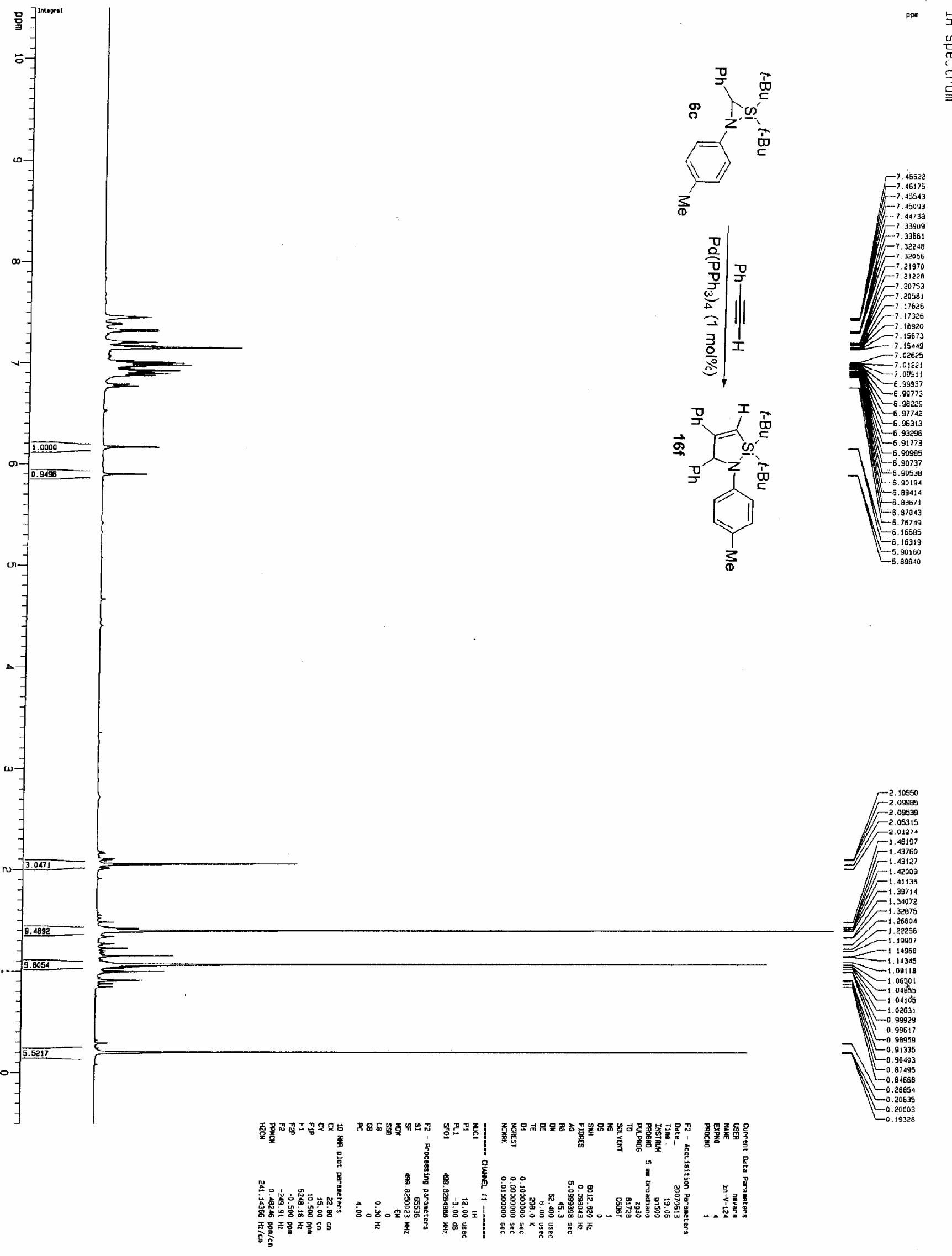




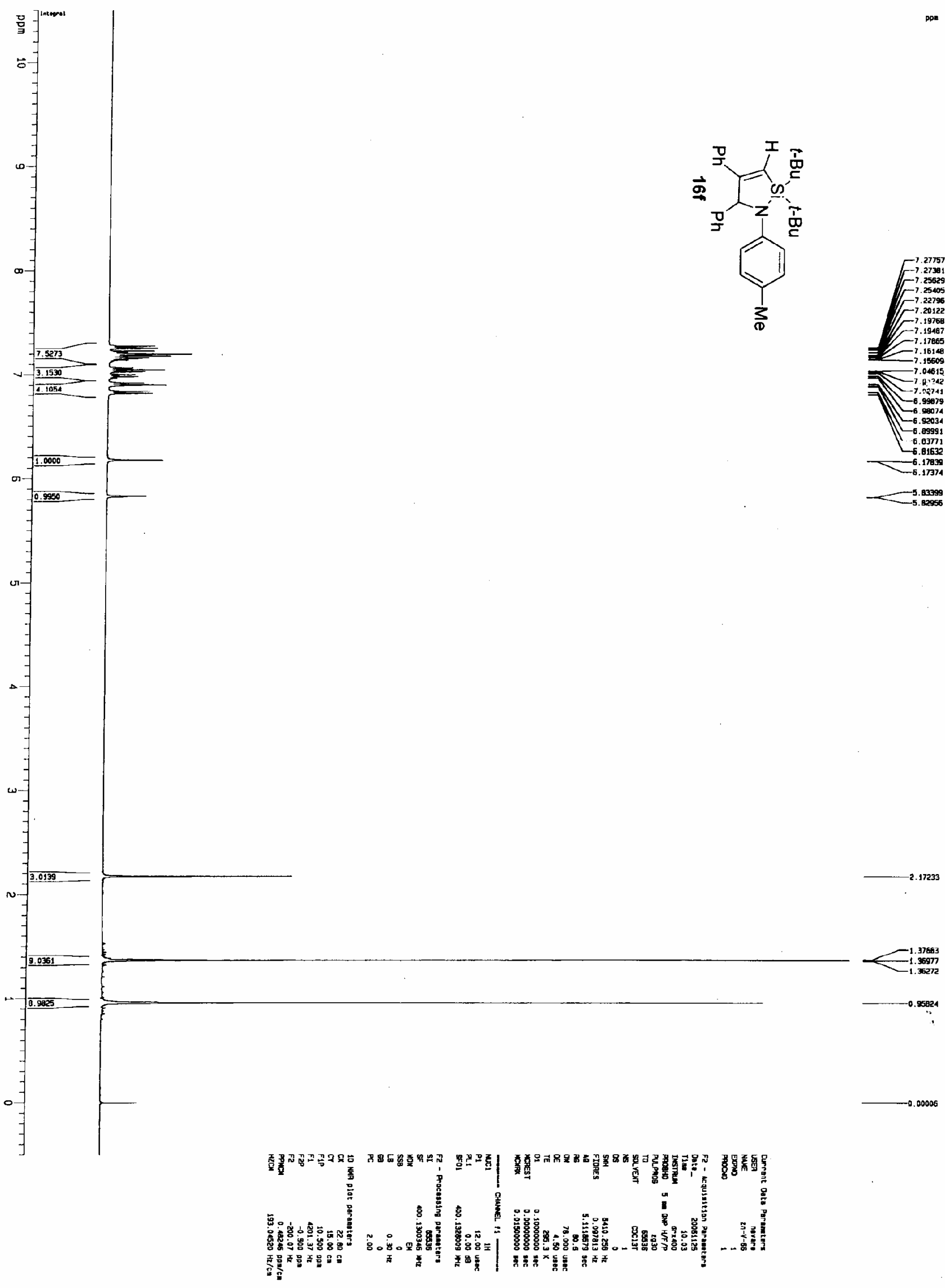



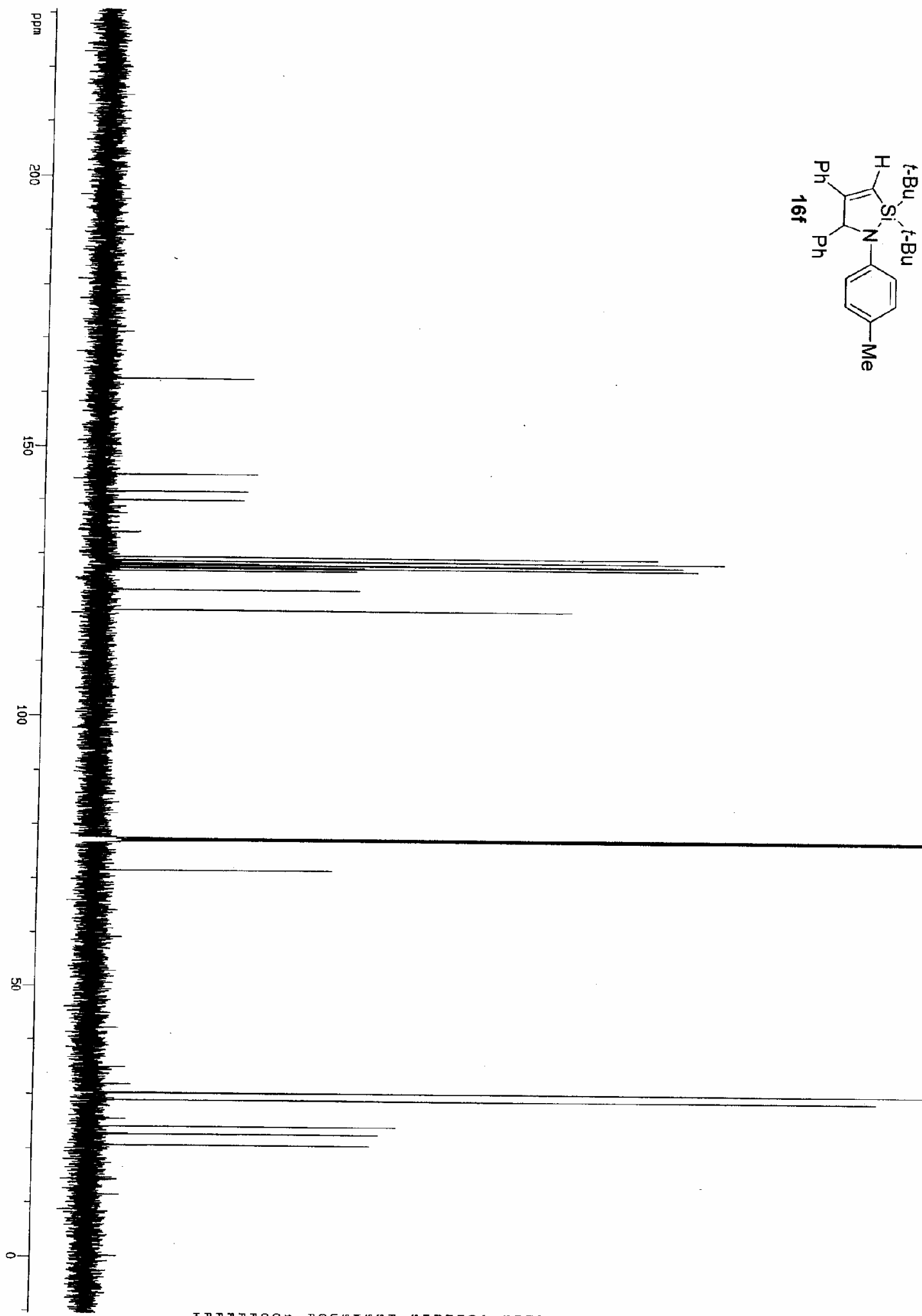

pope
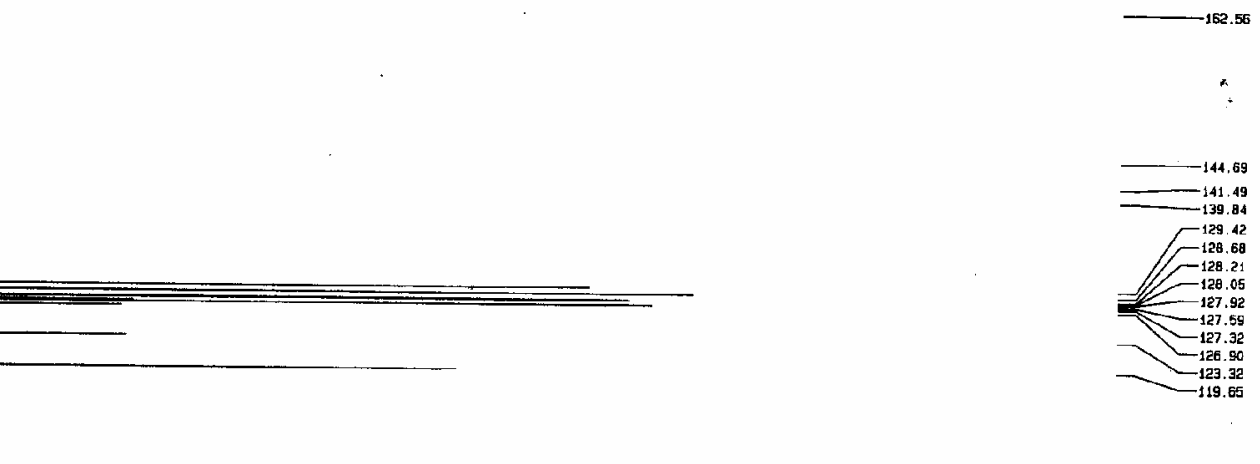


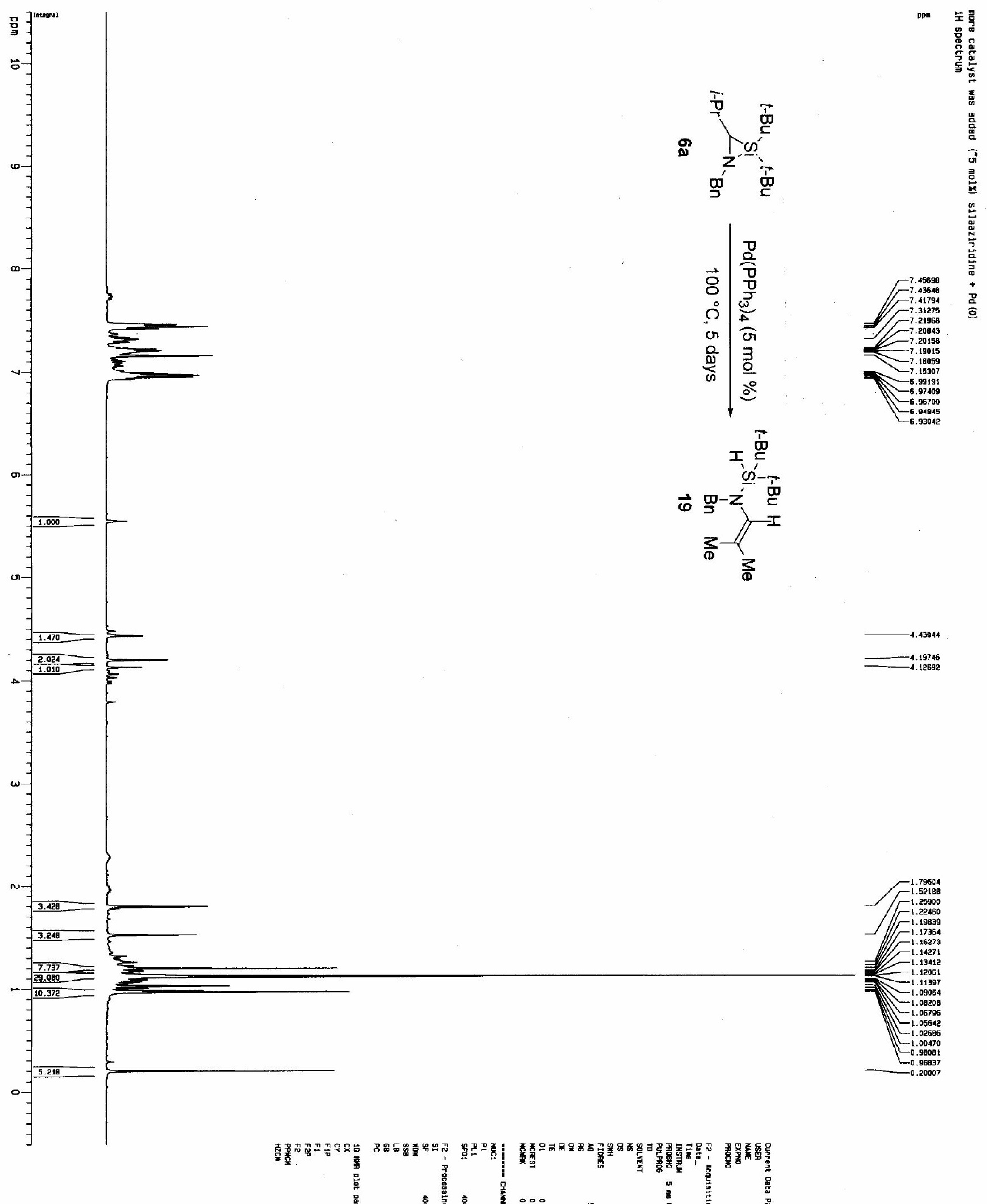

